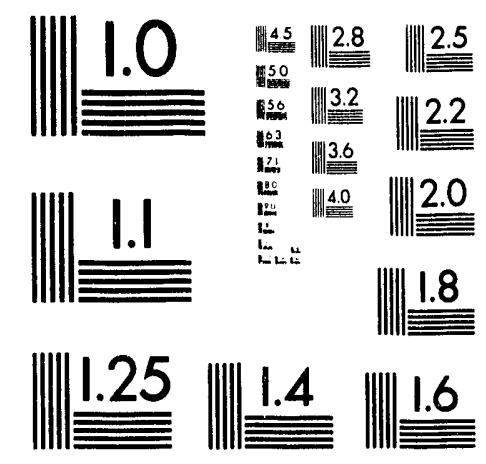



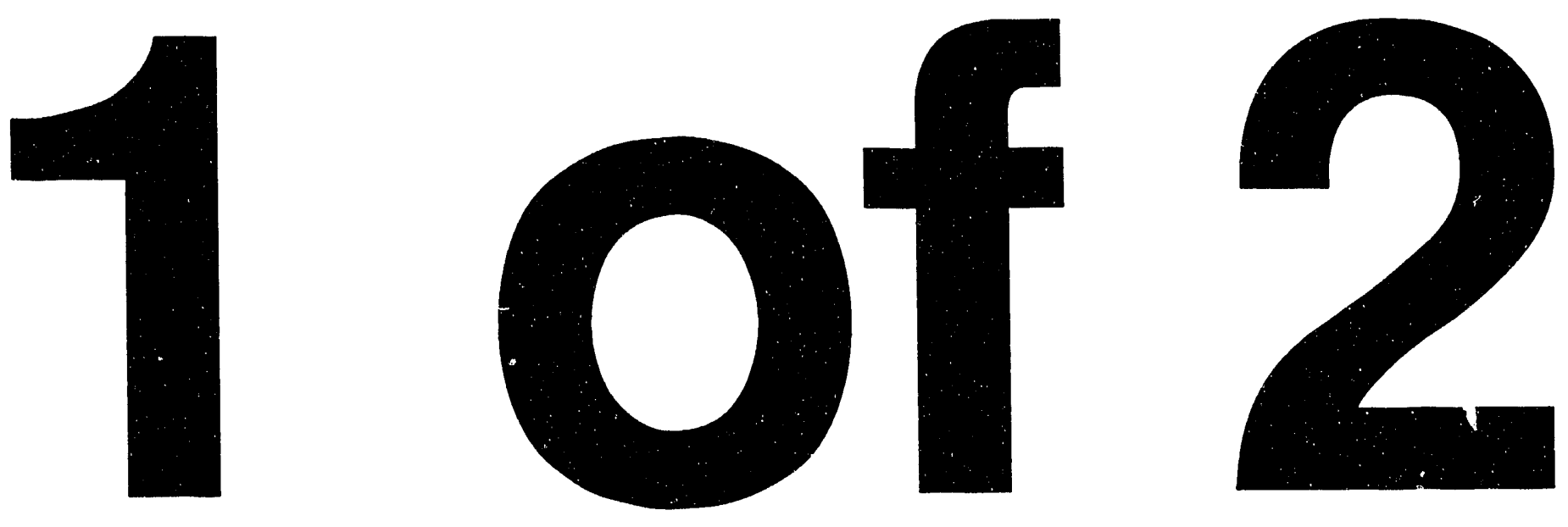
LA-UR- $93-3148$

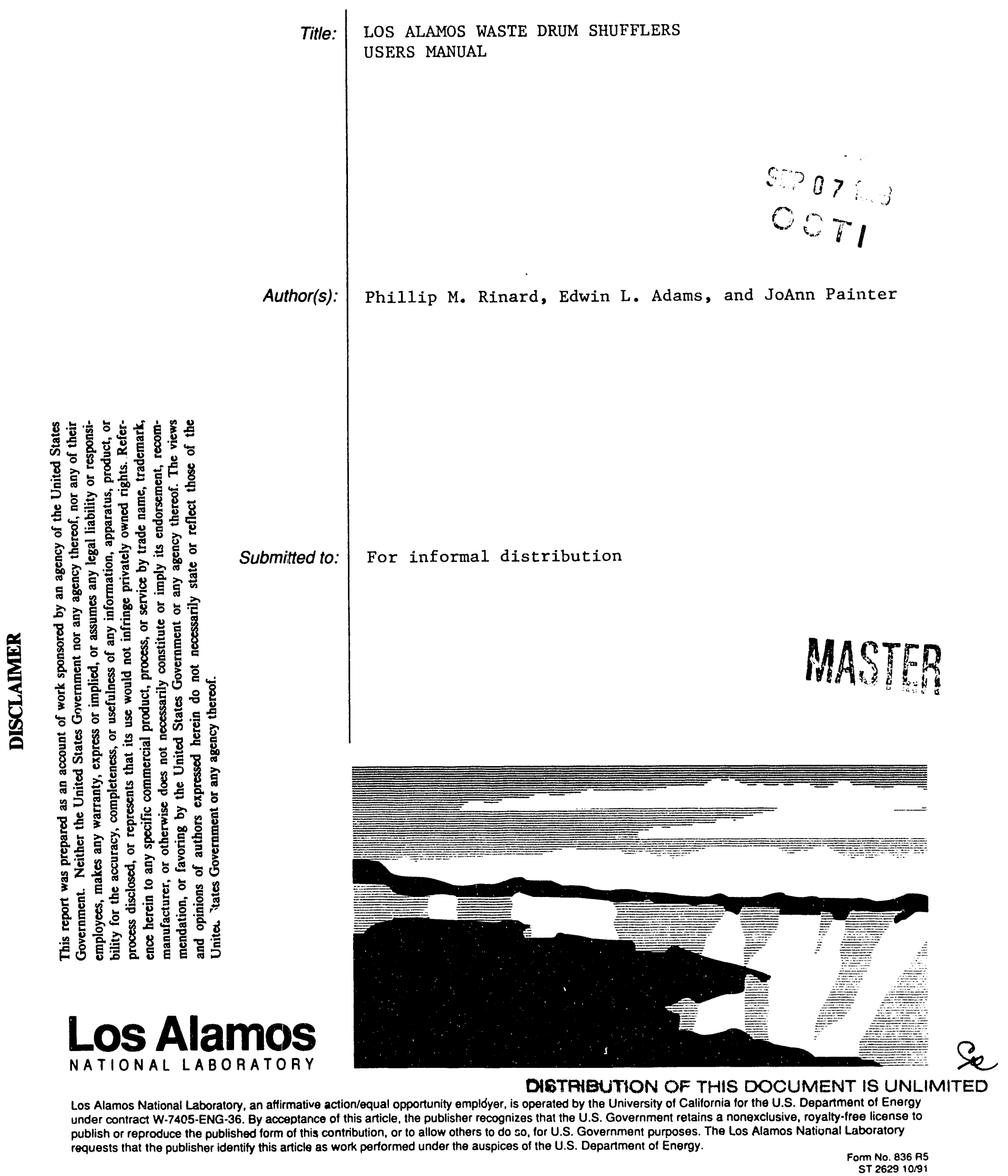




\section{LOS ALAMOS WASTE DRUM SHUFFLERS}

\section{USERS MANUAL}

Phillip M. Rinard, Edwin L. Adams, and JoAnn Painter

Safeguands Assay Group

Los Alamos National Laboratory

Los Alamos, NM 87545

August 24, 1993 


\section{CONTENTS}

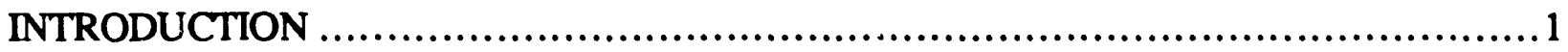

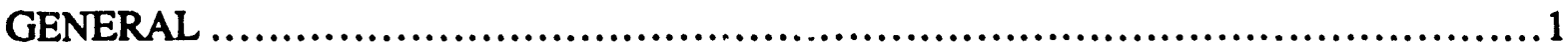

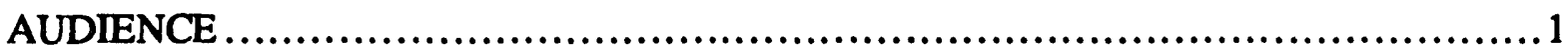

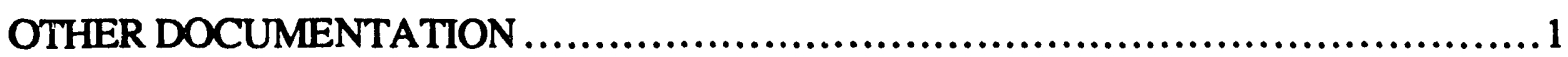

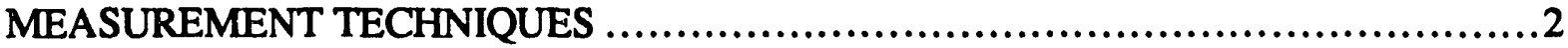

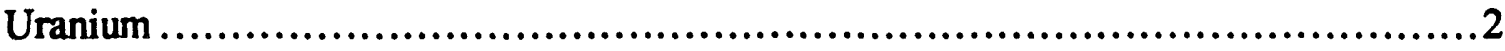

Plutonium and Other Fissile Materials ............................................5

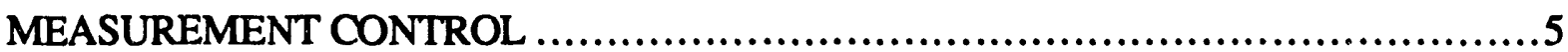

Internal.........................................................................

Procedural ...........................................................................6

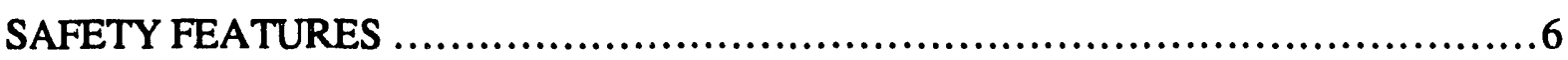

SHUFFLER HARDWARE OVERVIEW .......................................... 10

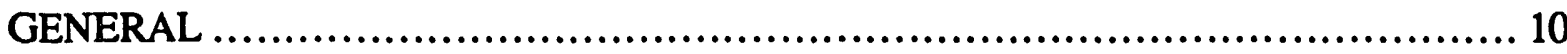

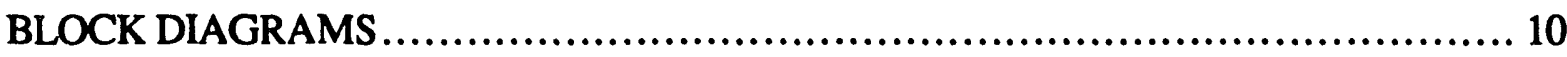

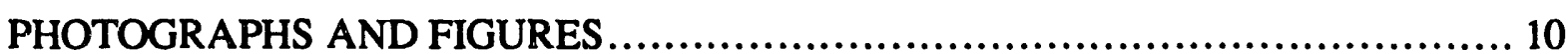

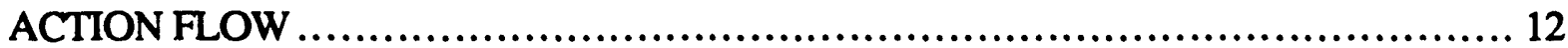

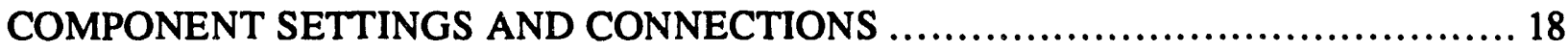

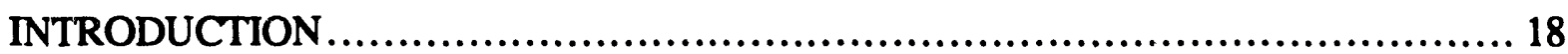

POWER CONDITIONER ........................................................... 18

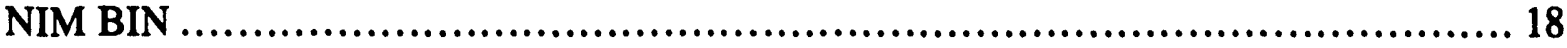

HIGH-VOLTAGE POWER SUPPLY ........................................... 18

Cable Connections ................................................................ 18

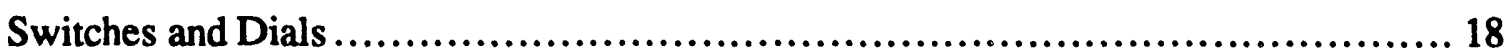

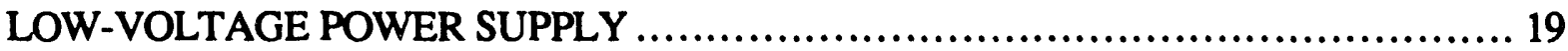

ABC SWITCH................................................................ 19

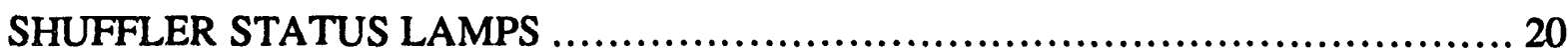

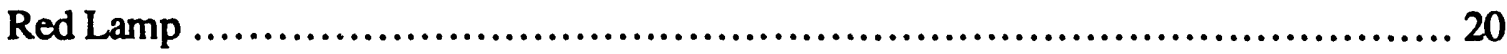

Green Lamp.................................................................. 21

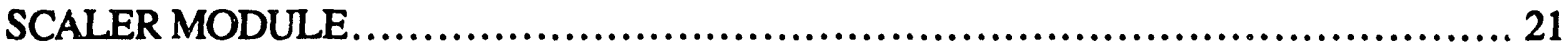

COMPUMOTOR INDEXER ......................................................... 22

Switch Settings ............................................................... 22

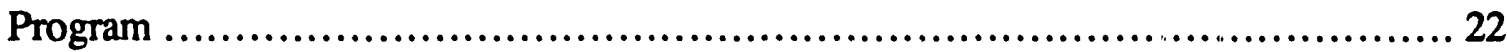

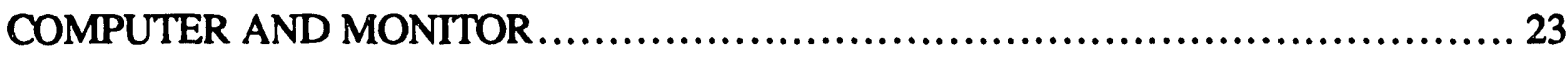

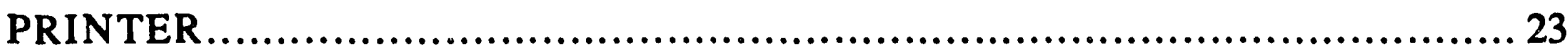




\section{CONTENTS}

(cont)

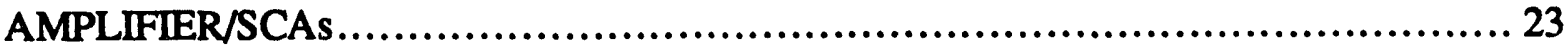

RESPONSE CHARACTERISTICS .............................................. 24

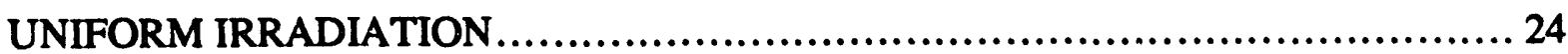

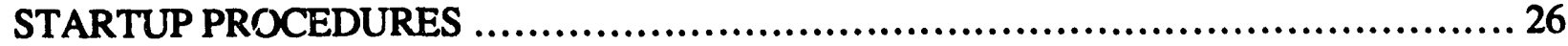

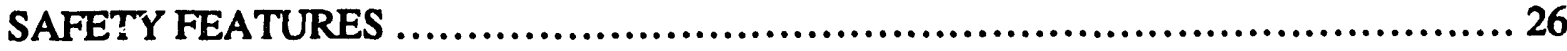

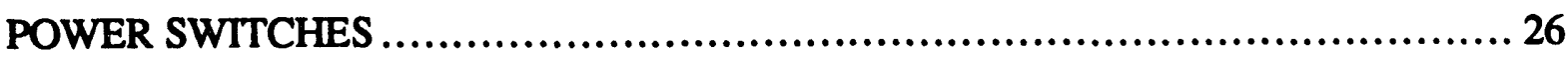

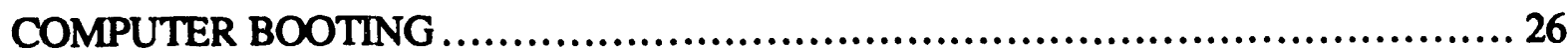

Configuration Files............................................................ 26

SIGN ON....................................................................... 27

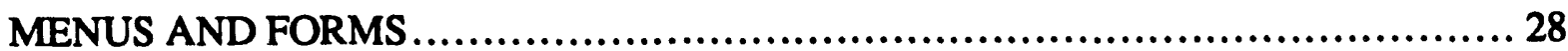

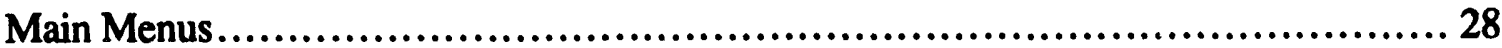

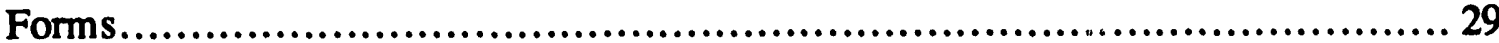

Lists.......................................................................... 30

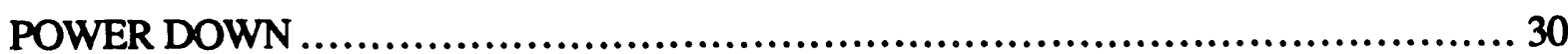

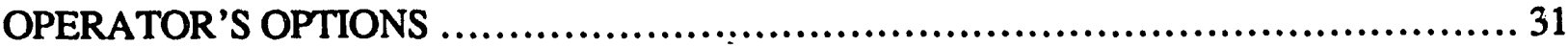

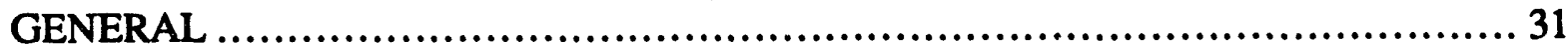

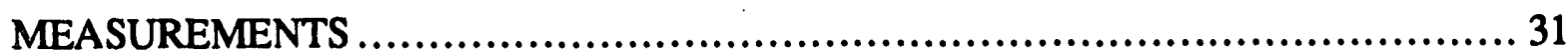

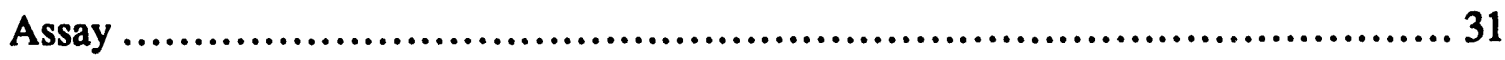

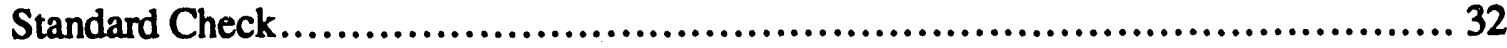

Bias and Precision Checks ..................................................... 33

Precision Check ............................................................... 33

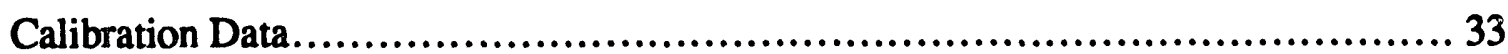

Plant Communications ........................................................... 34

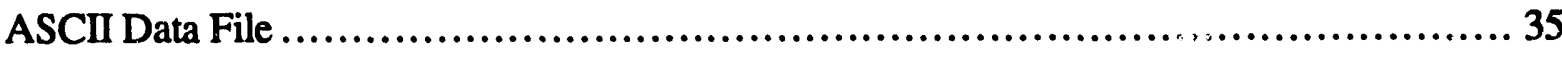

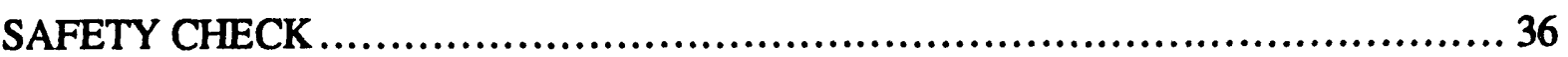

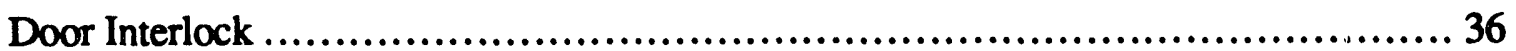

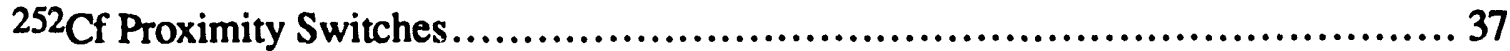

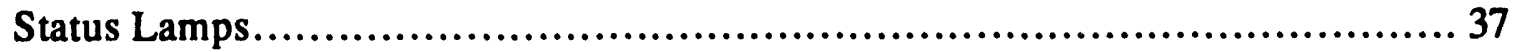

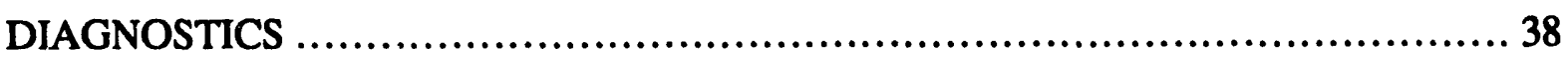

Test Door Proximity Switch ....................................................... 38

Test Cf Proximity Switches................................................... 38

Test Status Lamps ....................................................... 38

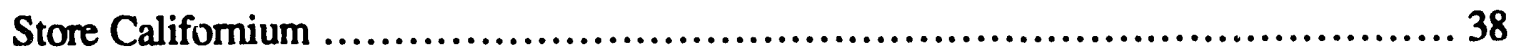




\section{CONTENTS}

(cont)

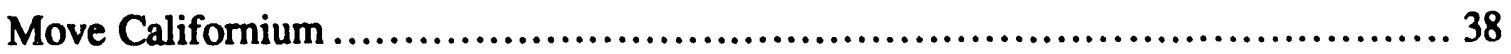

Move Californium to Test Position........................................................ 39

Count with No Californium Motion...................................................... 40

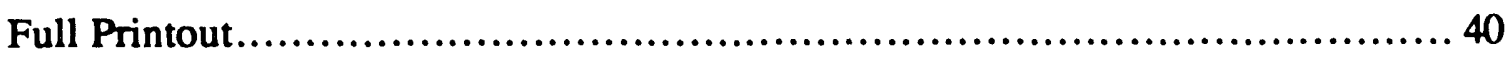

Print Last Results ................................................................ 41

Test Rotation Motor ............................................................. 41

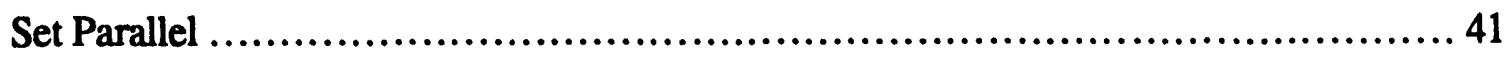

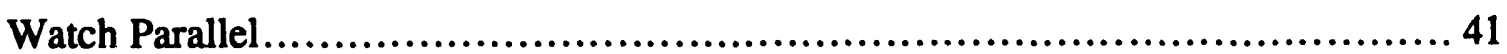

Download Indexer......................................................................... 42

Write ASCII Data .................................................................. 42

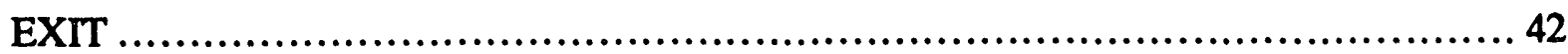

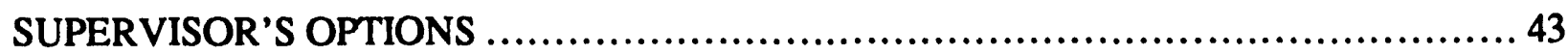

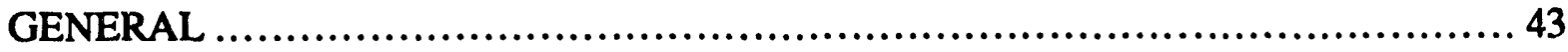

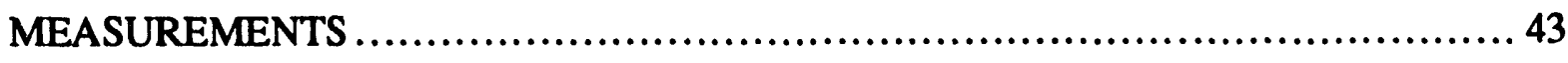

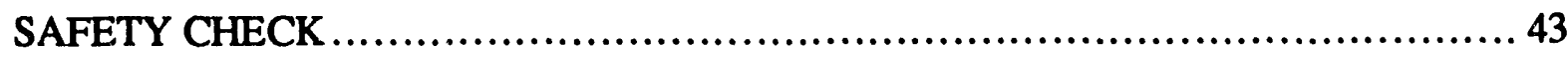

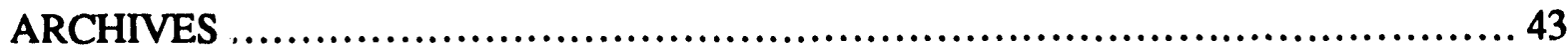

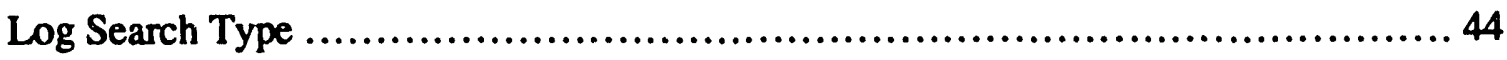

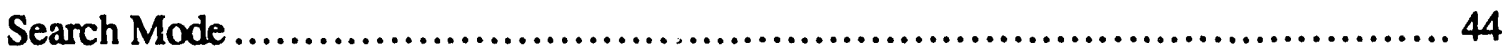

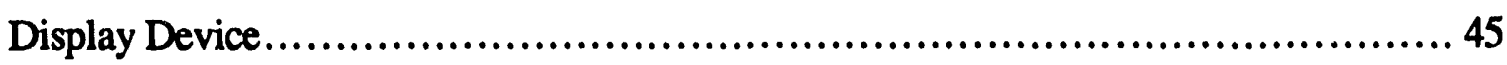

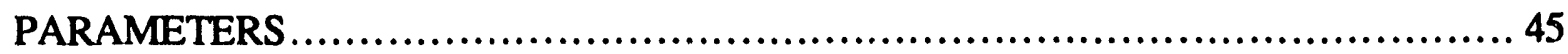

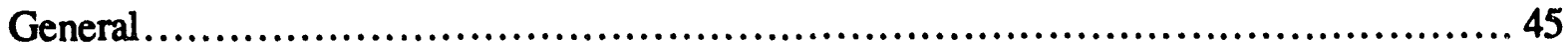

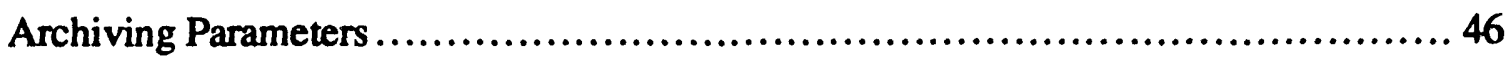

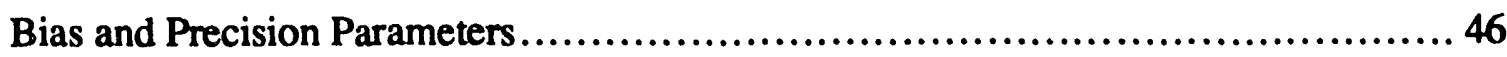

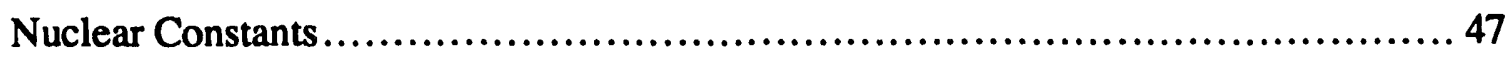

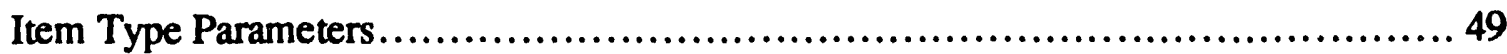

Standard Check Parameters ....................................................... 49

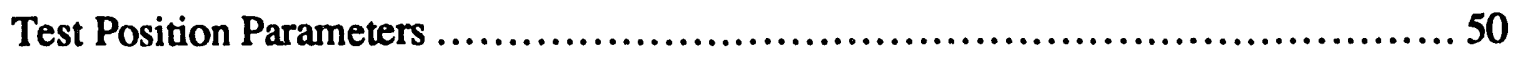

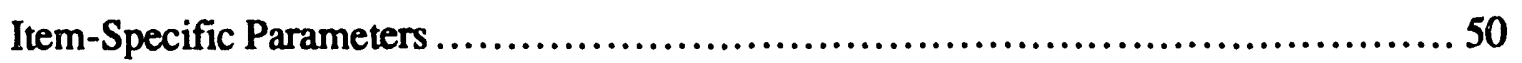

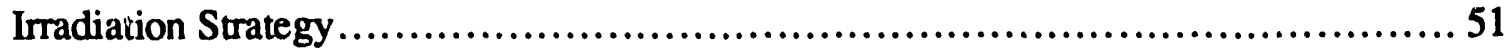

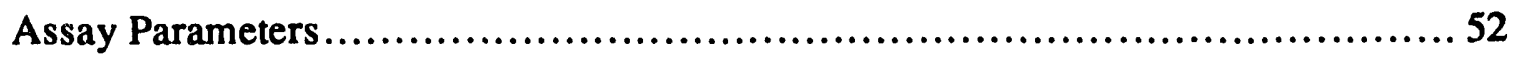

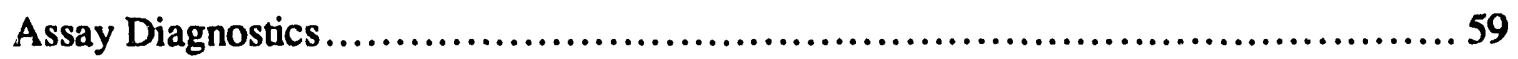

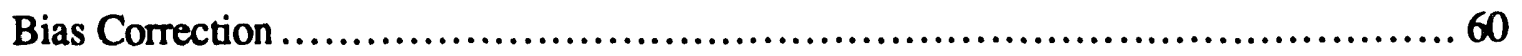

Calibration Parameters............................................................ 61 


\section{CONTENTS}

(cont)

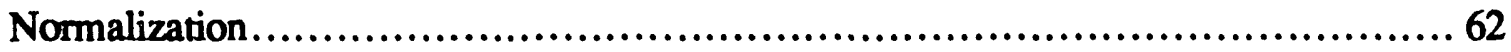

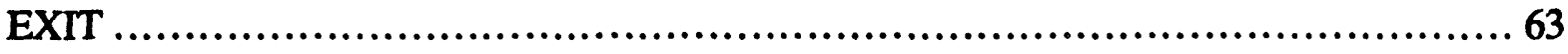

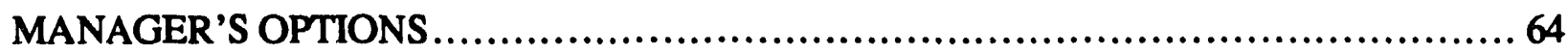

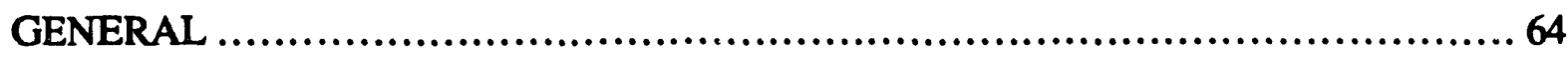

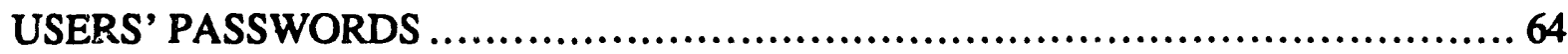

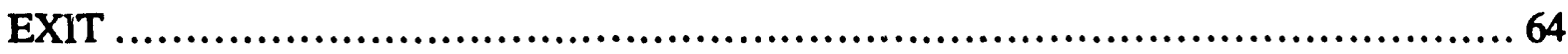

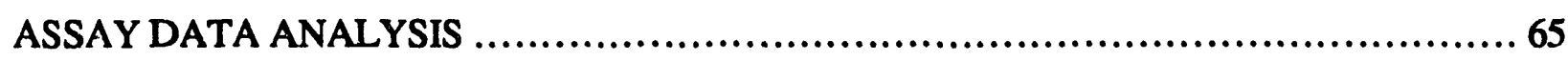

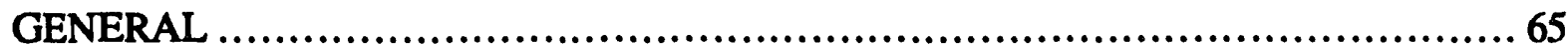

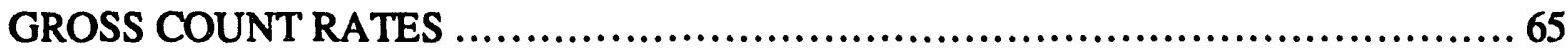

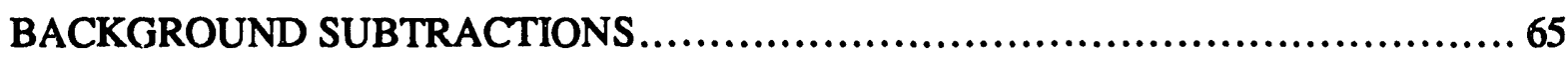

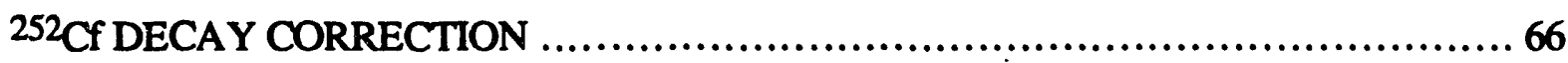

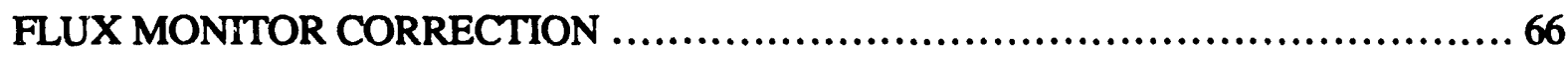

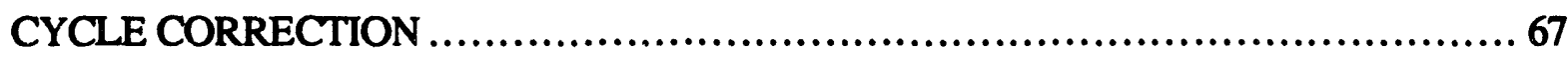

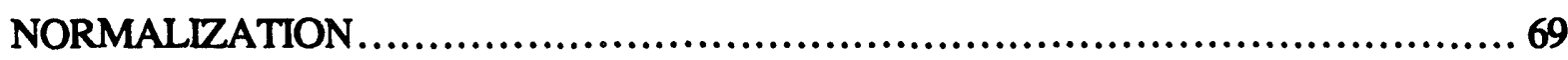

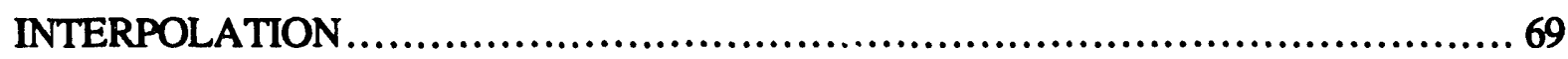

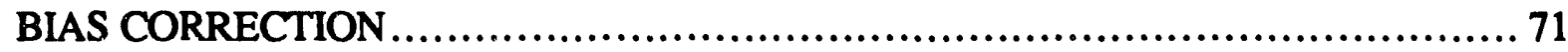

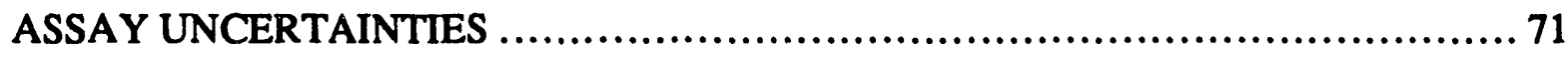

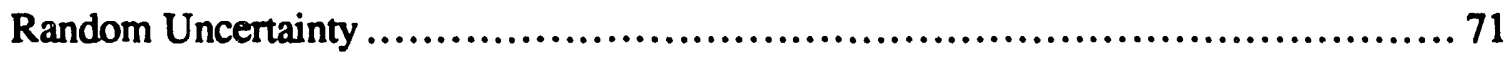

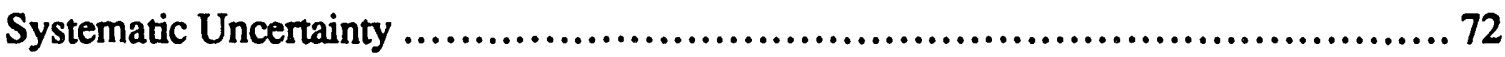

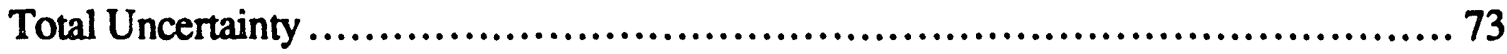

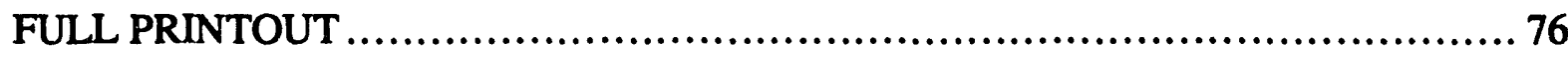

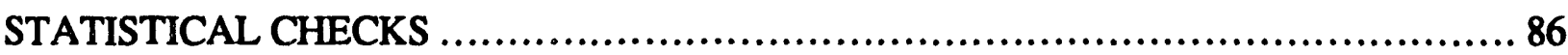

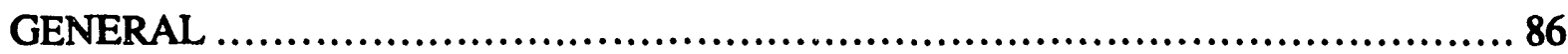

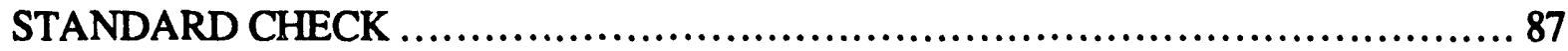

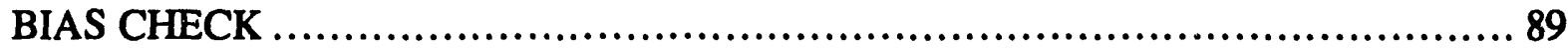

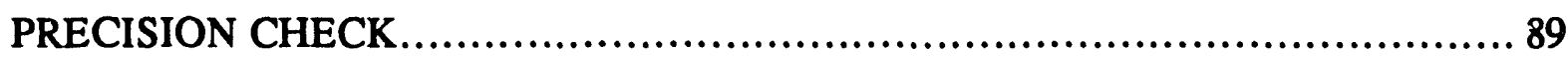

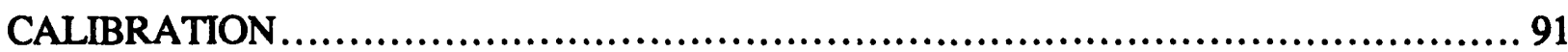

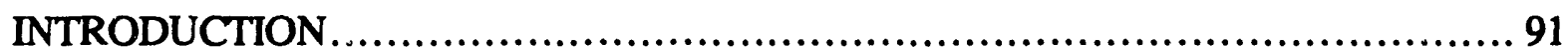

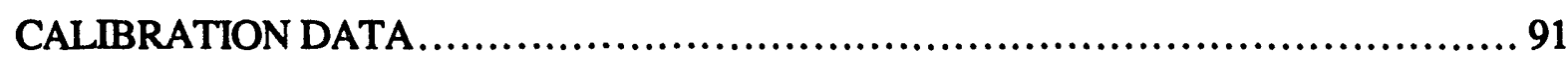

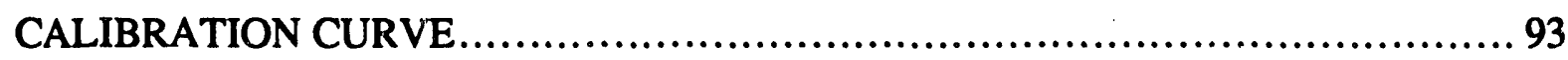

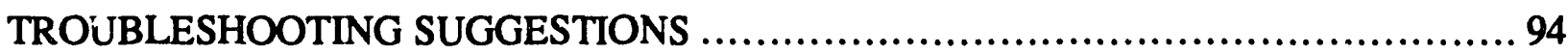

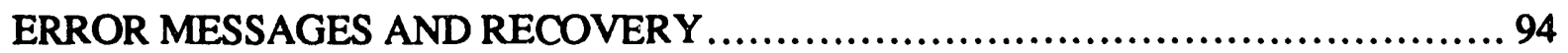

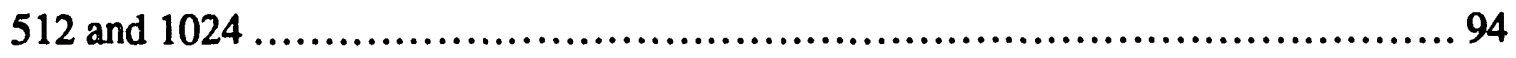




\section{CONTENTS}

(cont)

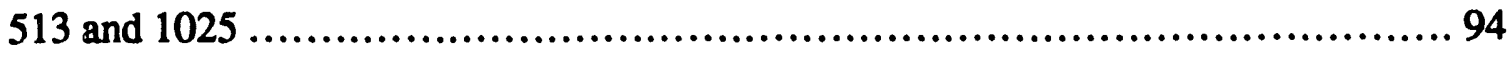

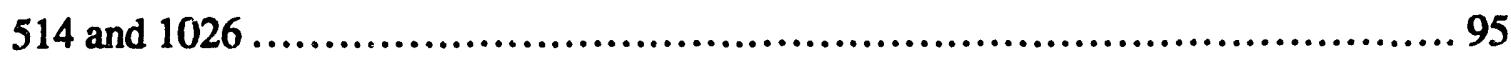

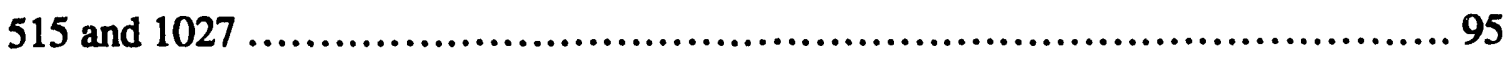

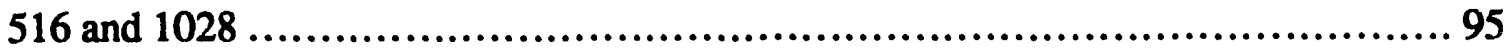

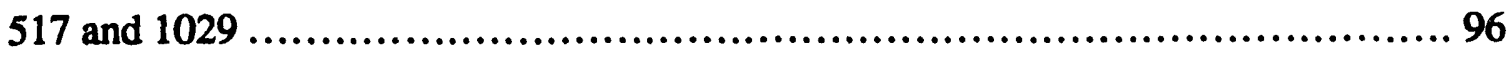

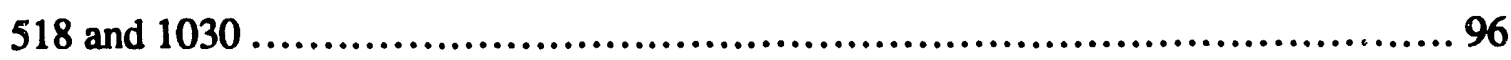

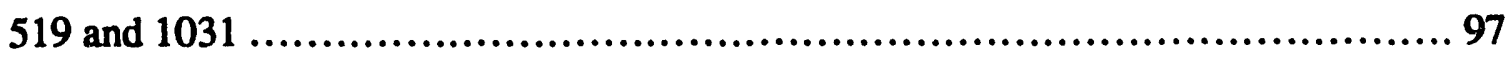

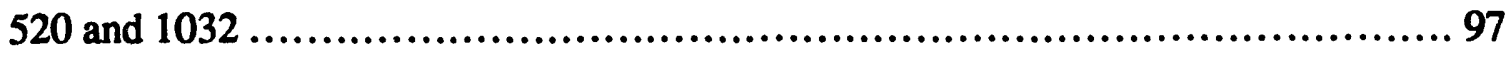

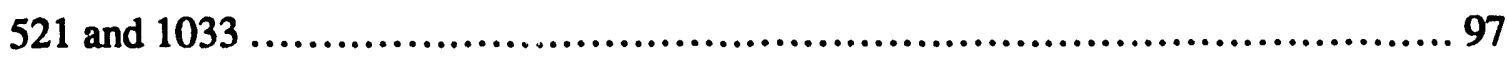

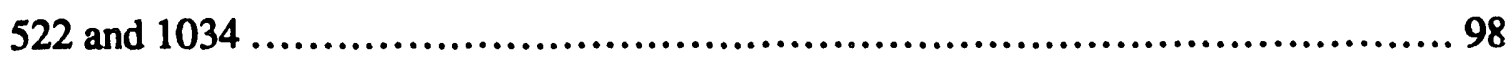

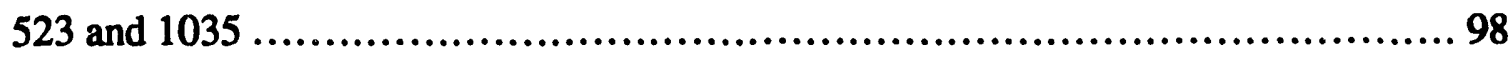

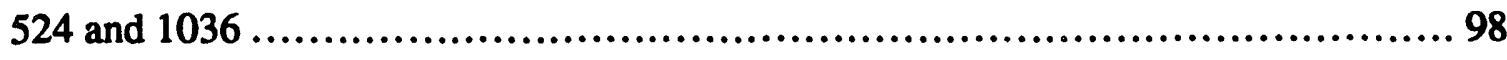

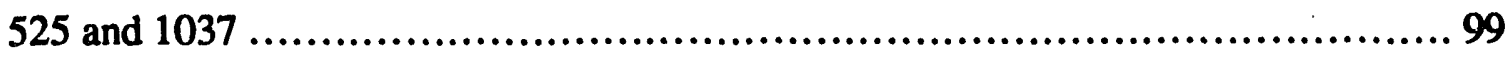

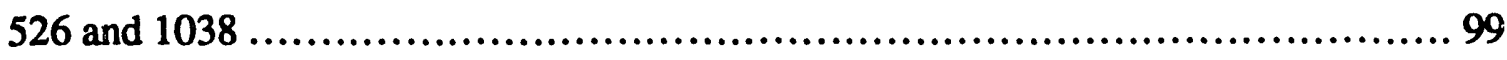

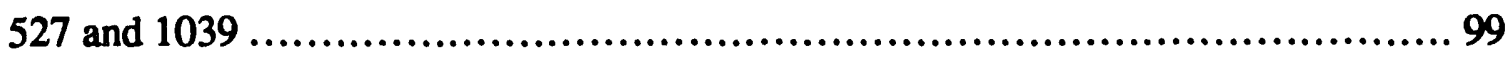

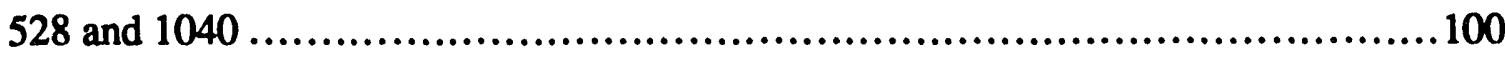

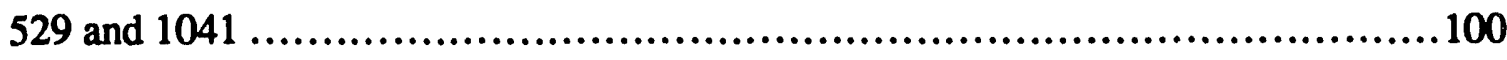

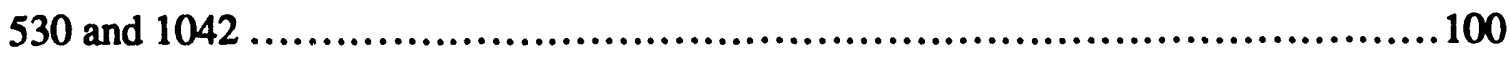

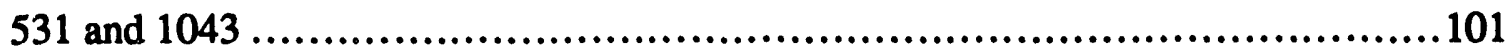

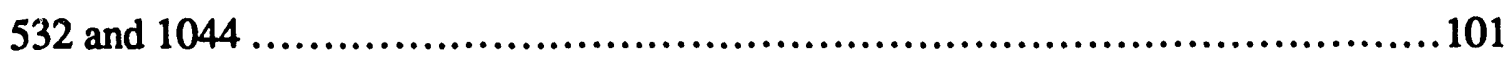

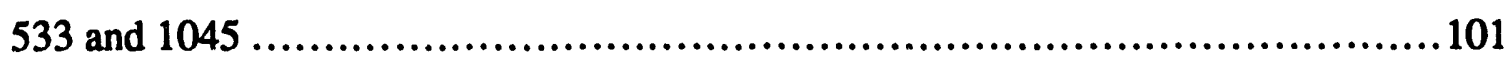

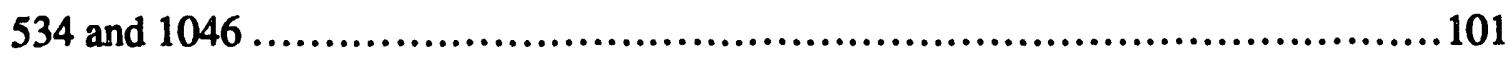

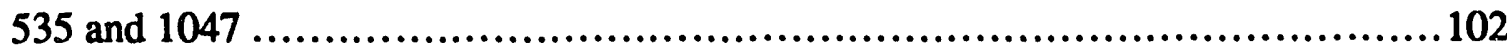

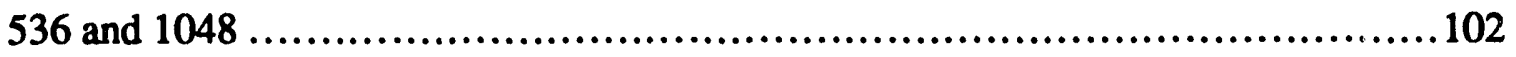

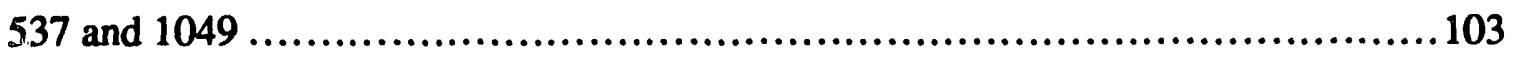

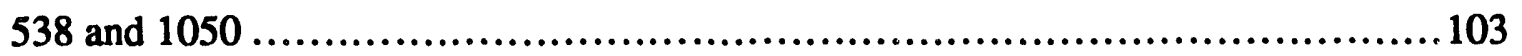

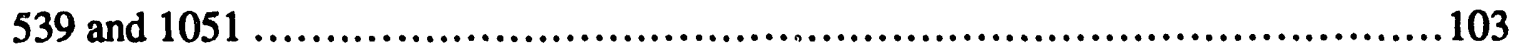

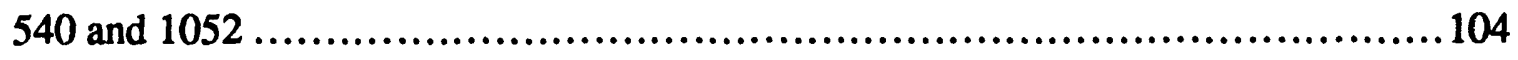

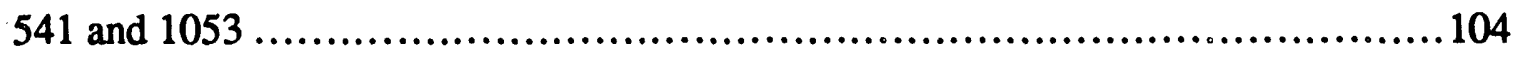

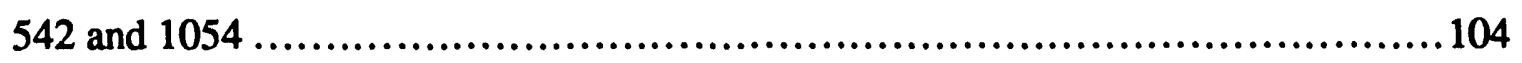

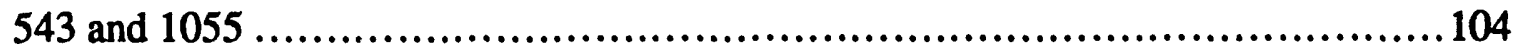

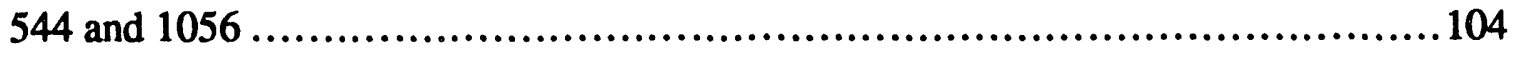

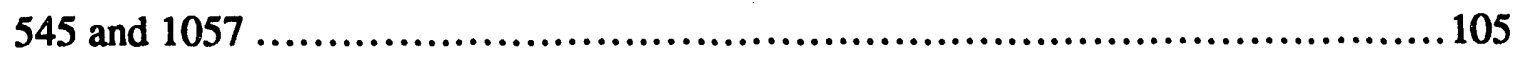




\section{CONTENTS}

(cont)

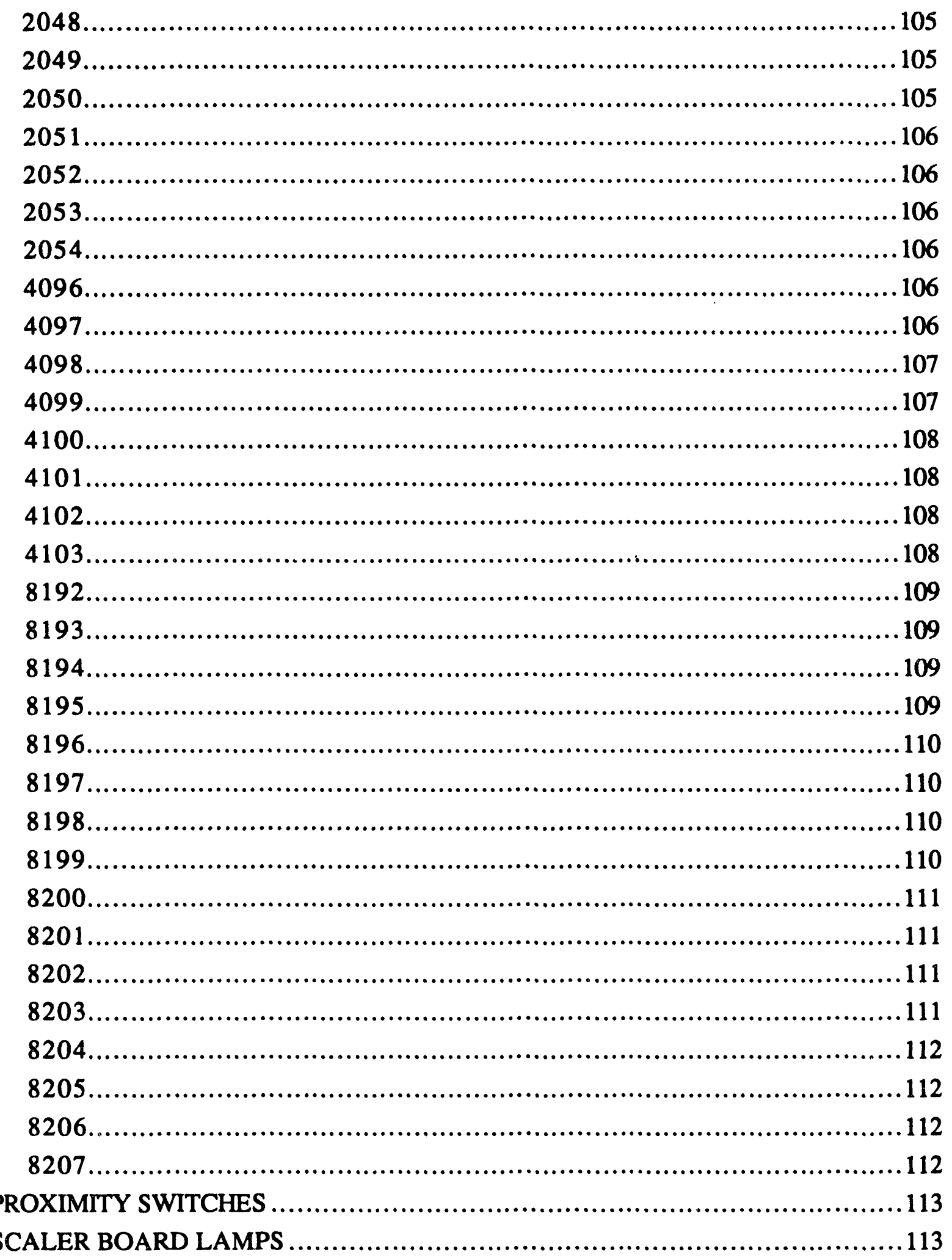




\section{CONTENTS}

(cont)

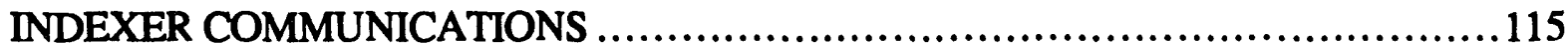

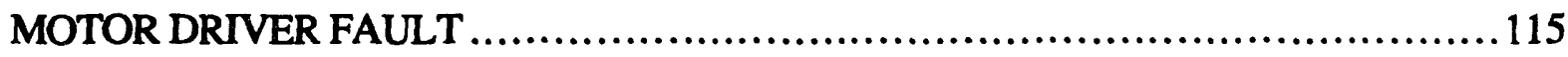

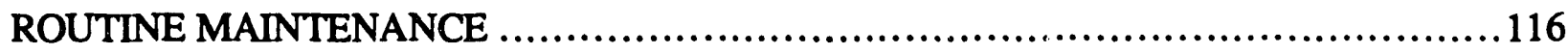

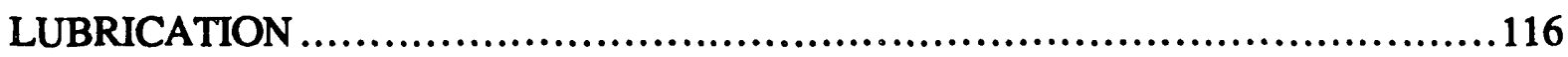

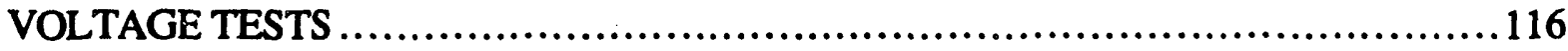

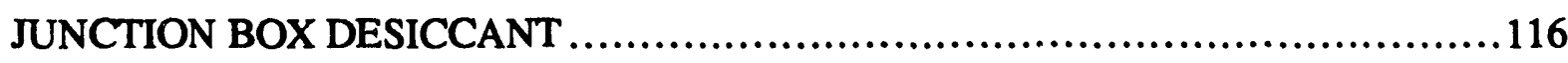

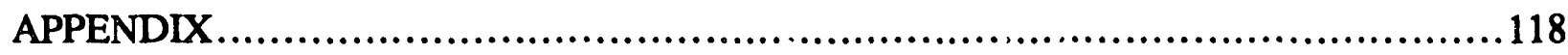

ASSAY TIMINGS AND ESTIMATION OF PARAMETERS ..........................118

The Purpose ........................................................................118

The Model......................................................................118 
GENERAL

AUDIENCE

OTHER DOCUMENTATION
This user manual describes the Los Alamos waste drum shufflers. The primary purpose of the instruments is to assay the mass of $235 \mathrm{U}$ (or other fissile materials) in drums of assorted waste. It can perform passive assays for isotopes that spontaneously emit neutrons or active assays using the shuffler technique (described below).

This user manual is intended for people who operate the shufflers.

Other manuals by Los Alamos and commercial manufacturers dealing with the shuffler are listed below.

1. Martin Marietta Portsmouth Waste Drum Shuffler Hardware Manual, Los Alamos

2. Martin Marietta Portsmouth Waste Drum Shuffler Software Manual, Los Alamos

3. Compumotor Model 3000 Controller User Guide, p/n 88-003181-05

4. Compumotor Operator's Manual, AX Drive, p/n 88006938-93

5. Compumotor A Drive User Guide, p/n 88-006937-04C

6. Compumotor Plus User Guide, Indexer/Driver Version, p/n 88-007488-02D

7. EG\&G Ortec Model 4001C Modular System Bin Operating and Service Manual

7. EG\&G Ortec Model 4002A Power Supply Operating and Service Manual

8. EG\&G Ortec Model 478 2-kV Bias Voltage Supply Operating and Service Manual

10. Micro Switch Installation Instructions for FM Approved DC FY Proximity Sensors, PK 82740

11. Catalog Sheets for Motion Technologies' Motor, Type 12 FGT, Model 6T15012001 
OTHER DOCUMENTATION $(\operatorname{con} t)$
MEASUREMENT TECHNIQUES

Uranium
12. Jomar Systems JSR-11 Electronics Package Operation Manual

13. IBM Personal System/2 Model 70386 Quick Reference and Reference Diskette

14. IBM Disk Operating System Version 3.30 Reference Manual

15. IBM Disk Operating System Version 3.30 Users Guide

16. IBM Disk Operating System Version 3.30 Quick Reference Card

17. 3+ Installation and Configuration Guide, $3 \mathrm{Com}$ Corporation, $\mathrm{p} / \mathrm{n}$ 2789-00

18. 3+ Administrator's Guide, 3Com Corporatior, Volume I, p/n 2788-01 and Volume II, p/n 4471-00

19. NetBIOS Supplement, 3Com Corporation, p/n 3254-00

20. 3+ Menus Reference Guide, 3Com Corporation, $\mathrm{p} / \mathrm{n}$ 1963-01

21. 3+ Messages Reference Guide, 3Com Corporation, $\mathrm{p} / \mathrm{n}$ 4472-01

22. 3+ Share User Guide, 3 Com Corporation, $\mathrm{p} / \mathrm{n}$ 1694-01

23. 9570 Wedge Reader Operator's Guide, Intermec, $\mathrm{p} / \mathrm{n} 050682$ Version B

24. 1620A Laser Scanner Operator Guide, Intermec, p/n 045205

25. 9570 Wedge Reader Quick Reference Card, Intermec, p/n 047857

The shuffler measurement technique for uranium (or any other fissile material) relies on counting delayed neutrons emitted seconds to minutes after fission events induced in the drums by neutrons from an irradiating $252 \mathrm{Cf}$ source. The ${ }^{252} \mathrm{Cf}$ source irradiates a drum for a few seconds and is then withdrawn to a shielded position so the delayed neutrons can be counted for a few seconds. This process is repeated, 
Uranivm

(cont) shuffling the source to and from the drum, until the desired number of delayed neutions has been counted. The measured count rate is correlated with the $235 \mathrm{U}$ mass in the drum through calibration with standard drums. Other isotopes of uranium, also fission, and ${ }^{238} \mathrm{U}$ is generally the next most important contributor to the delayed neutron count; when different enrichments are being measured, the effect of enrichment must be included through calibration or a calculated correction.

Much more technical information on the theory of shufflers is presented in the Los Alamos National Laboratory report LA-12103 (May 1991) by P. M. Rinard, "Shuffler Instruments for the Nondestructive Assay of Fissile Materials." A. brief overview of the capabilities of shufflers for 55-gal. drums is presented in the Los Alamos National Laboratory Application Note, "The ${ }^{252}$ Californium Shuffler," (March 1990).

A user commands the instrument through the keyboard of a computer. In the electronics rack are high- and low-voltage power supplies for the neutron detector tubes, a 12-channel scaler for timing and counting pulses from amplifiers connected to the neutron detectors, the stepping motor indexer, a lamp controller, a printer, and assorted electronics to condition signals to and from the computer.

A drum to be assayed is placed on a wheeled cart and rolled into the assay chamber. After a retaining peg is placed in the chamber and the door is closed, the source can be moved into the assay chamber under control of the Indexer upon command by the computer. The computer notes the state of a proximity switch in the dnor and will never ask to move the source if the door is open; if told to move the source when the door is open, the code inside the Indexer will not 
Uranium

(cont) do so because it too checks the state of the door's proximity switch.

A stepping morior turns gears that drive a cable attached to the source. Proximity switches watch for overtravel of the cable and shut down the stepping motor when an overtravel occurs. The reverse overtravel sensor also indicates when the source is stored in the center of the storage chamber.

The stepping motor Indexer has parallel input and output bits that are used to transmit information between the computer and peripherals. The states of the proximity switches are read by the computer from the input port and the status lamps are set through the output port. The Indexer has its own internal code consisting of several types of operation that can be accessed individually by the shuffler's computer; examples are moving the source to or from a drum, scanning the source along a drum, and setting or reading parallel bits. Communication between the computer and the Indexer is over a serial port.

When a measurement is commanded, the appropriate portion of the computer's code sets the status lamps on the shuffler body, clears the scaler's channels of counts, checks proximity switches for the ${ }^{252} \mathrm{Cf}$ source's cable and the door, and if everything is in order, proceeds with the measurement.

A drum is surrounded by eight banks of large delayedneutron detector tubes and two small flux monitor tubes that respond to delayed neutrons from the drum when the source is returned to the storage chamber and neutrons from the ${ }^{252} \mathrm{Cf}$ when it is irradiating the drum, respectively. Each bank of tubes is connected to an amplifier board built around the AmpTek amplifier integrated circuit. The signals from the amplifiers are counted by the 12-channel scaler module. 


\section{Uranium \\ (cont)}

Plutonium and Other Fissile

Materials
When the computer's clock estimaces that a counting time period is finished, the 12-channel scaler is read. Channel 0 gives the precise count time to the nearest millisecond and other channels provide the counts in the eight delayed-neutron o itector banks and the two flux monitors. These raw data are used by the data analysis portion of the code to generate an assay result.

Plutonium, UF6, or other spontaneous emitters of neutrons can be assayed through passive neutron counting. These neutrons originate from spontaneous fissions or $(\alpha, n)$ reactions. The shuffler's neutron detection electronics is used but the total signal is routed to a shift register electronics package (Jomar Systems JSR-11). The shuffler's software is not used for passive counting; a separate computer program controls the data collection and analysis.

The use of the shuffler electronics for passive assays will not be described further in this manual.

\section{MEASUREMENT CONTROL}

Internal
As part of every assay, some internal checks are made automatically to ensure that the shuffler's components are operating properly.

If any bank of neutron detectors has no counts, there has been a problem with that bank or the scaler board. There are eight banks counting delayed neutrons and two individual flux monitor detectors.

Ratios of counts from detector banks are computed and compared to expected ranges of values in a parameter file of the computer. The ratios must be within the range in the parameter file for the assay to be considered "normal." The same is true for the ratio of the two flux monitors, although 
Internal

(cont) the use of these monitors during assays is optional (specified in the parameter file).

If the serial communication link to either the scaler board or the Compumotor Indexer is lost, the shuffler's computer informs the user through the video monitor and assays cannot proceed until the problem is fixed.

The Compumotor Indexer performs its own internal checks on its operation and signals the shuffler's computer if it finds a problem in the stepping motor system.

Measurement control options are available to the user through the menu on the video monitor.

A Precision and Bias Check may be performed by placing a standard drum of known 235U mass in the shuffler and assaying it repeatedly. The average assayed mass and the precision of the assays are compared to ranges of values in the parameter file. If either check is failed, the whole test must be repeated. A second failure requires the assistance of a supervisor before further assays can be taken.

The precision portion of the above check may be performed without the bias portion. This allows the use of a nonstandard drum whose $235 \mathrm{U}$ mass is not well known. The precision is calculated but not compared to any value in the parameter file.

A Standard Check is required of the operator on a schedule specified in the parameter file. An assay is first made of an empty assay chamber, the result should be below the specification in the parameter file. A second assay is then made on a standard drum, with the result again being compared to a standard value in the parameter file. Failure to pass these 
Procedural

(cont)

\section{SAFETY FEATURES}

tests after two attempts requires the assistance of a supervisor.

The only unusual hazard associated with a shuffler comes from the ${ }^{252} \mathrm{Cf}$ source. This isotope emits gamma rays and neutrons spontaneously with a half-life of 2.64 years. It is doubly encapsulated in stainless steel capsules that have been used as standard containers for many years after thorough testing by Oak Ridge National Laboratory and Westinghouse Savannah River Company.

The bulk of the shuffler's body is shielding for the ${ }^{252} \mathrm{Cf}$ radiation. The shielding has been selected for a nominal $600 \mu \mathrm{g}$ of ${ }^{252} \mathrm{Cf}$. The dose rate from the source at a given location depends on the source and measurement positions and whether or not averages are taken over position and/or time.

The initial recommended size of a new ${ }^{252} \mathrm{Cf}$ source is $600 \mu \mathrm{g}$. If a person were $1 \mathrm{~m}$ from an exposed source of this size with no intervening shielding whatsoever, the dose rates received would be $1404 \mathrm{mrem} / \mathrm{hr}$ from neutrons, $84 \mathrm{mrem} / \mathrm{hr}$ from gamma rays, and thus a total of $1488 \mathrm{mrem} / \mathrm{hr}$. If a person were $2 \mathrm{~m}$ from this size source for $10 \mathrm{~s}$, the dose received would be $(1488 \mathrm{mrem} / \mathrm{hr})(1 \mathrm{~m} /$ $2 \mathrm{~m})^{2}(10 / 3600 \mathrm{hr})=1.03 \mathrm{mrem}$. The shuffler's shielding reduces this dose rate to values indicated in the next paragraph.

During an assay the source shuffles between its normal storage position and an assay position in the rear of the assay chamber. The source is secured to a flexible cable, which in turn is moved by a stepping motor and gear. If the shuffler's door were to be opened while the source is in the assay position, there could be little shielding between the source 


\section{SAFETY FEATURES}

(cont) and the person. A proximity switch on the door is provided that senses that the door is open or closed. An electronic interlock and the software will not allow an assay to begin unil the door is closed. If the door is opened during an assay, the source is automatically withdrawn into its shield and the assay is terminated. As indicated by the example in the preceding paragraph, the dose received by someone opening the door will be much less than 1 mrem. As soon as the door is opened even a crack, the sciurce will start moving away from the door and into the storage chamber. By the time the door is opened enough for someone to stand by the open end of the assay chamber, the source will be within its shield.

The position of the ${ }^{252} \mathrm{Cf}$ source is controlled by the Compumotor Indexer and the shuffler's computer. Three proximity switches provide information on key positions of the source by detecting the free end of the cable.

One proximity switch signals when the source is in its "stored" position at the center of the storage chamber. This switch is checked many times during an assay for safety reasons and also to ensure that the source is away from the assay chamber while delayed neutrons are being counted. Should the stepping motor system not return the source to this stored position to within a small fraction of an inch, the Indexer will automatically cause the motor to take the few steps required to position the source properly.

A second proximity switch is just beyond the other extreme position taken by the source while it irradiates a drum. This is a "forward overtravel" switch and if the source's cable uncovers this switch, the Indexer immediately stops the stepping motor. 
SAFETY FEATURES (cont)
The third proximity switch is slightly beyond the store position proximity switch and serves as a "reverse overtravel" switch. If the source's cable uncovers this switch, the Indexer immediately stops the stepping motor. The proximity switches are sensed by the Compumotor Indexer and by the shuffler's computer. The Indexer will respond to the overtravel signals independently of the computer, it automatically stops its own motion if an overtravel occurs. The Indexer code responds to the door and store switches, but the code must be running (which is necessary to operate the shuffier). The shuffler's computer monitors all these switches and takes appropriate actions based on their states. 
GENERAL

BLOCK DIAGRAMS
The shuffler's hardware has three separate components. Ali of these components are discussed in more detail later in this SHUFFLER HARDWARE OVERVIEW section of the manual.

1. The first component is the shuffler body, which consists of these three sections:

a. a storage chamber with the stepping motor drive system;

b. an assay chamber containing the neutron detector tubes, signal amplifiers, and turntable rotation mechanism; and

c. doors giving access to the assay chamber.

2. The second component is the electronics rack with the high-voltage and low-voltage supplies, signal conditioning modules with status lamps, the stepping motor Indexer, rotator driver and controller, and a power conditioner.

3. The third component includes a computer and printer on a table.

Electrical drawing $68 \mathrm{Y}-155750$ D200 is a block diagram showing the electrical components and their interconnections.

PHOTOGRAPHS AND FIGURES Figure 1 is a photograph of the shuffler's body as seen from the front. Figure 2 shows details of the driving mechanism for the ${ }^{252} \mathrm{Cf}$ source. Figure 3 is a photograph from the front with the doors open. 


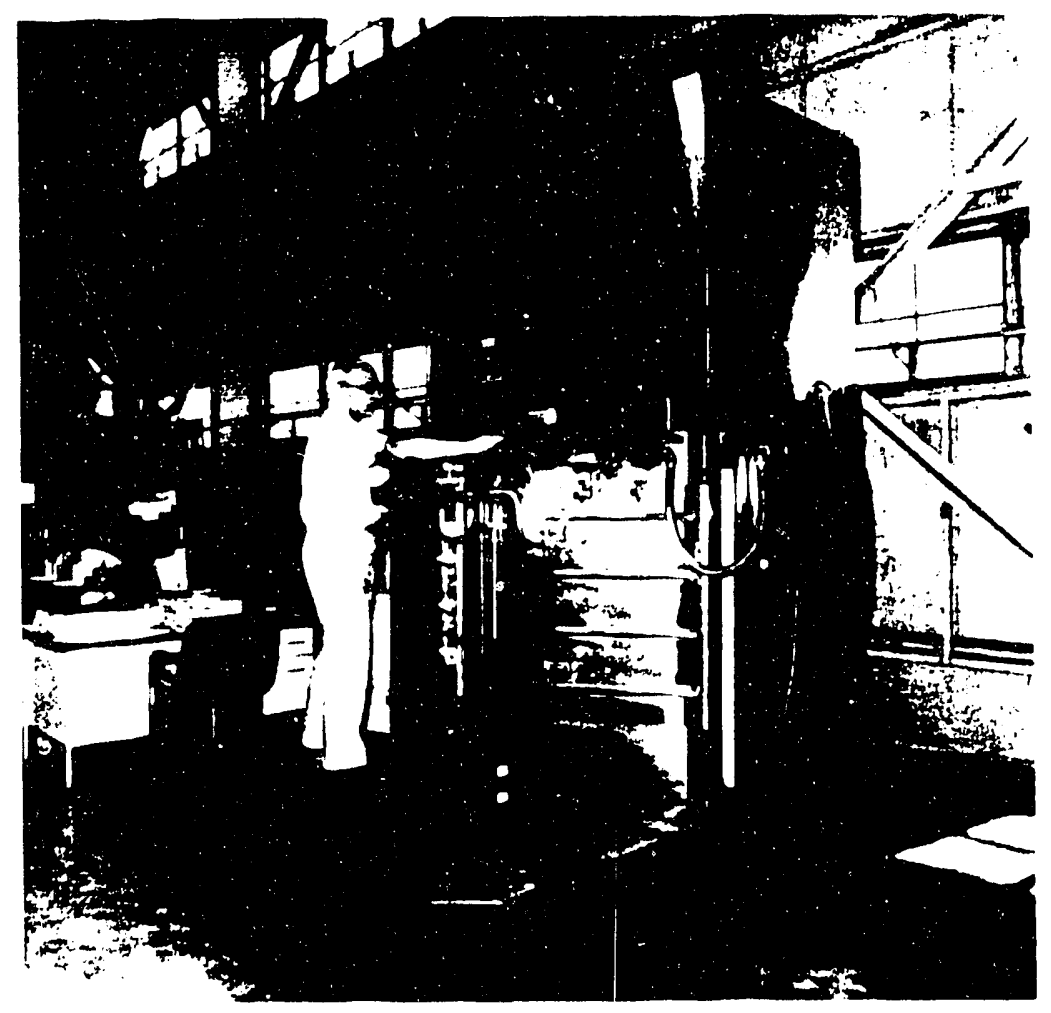

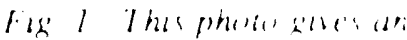
onerve's of the shuffle" bead line

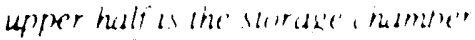

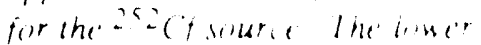

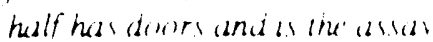

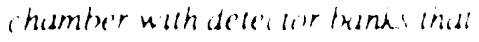
surround a drum Belesu the desas

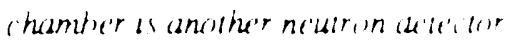
bank and the mer humbsm bre ridte' a drum during an asisd

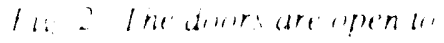

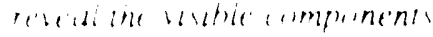

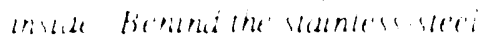

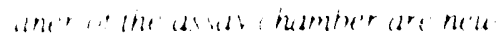

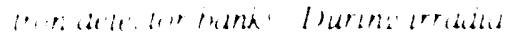

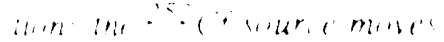

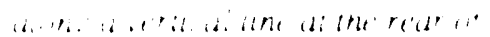

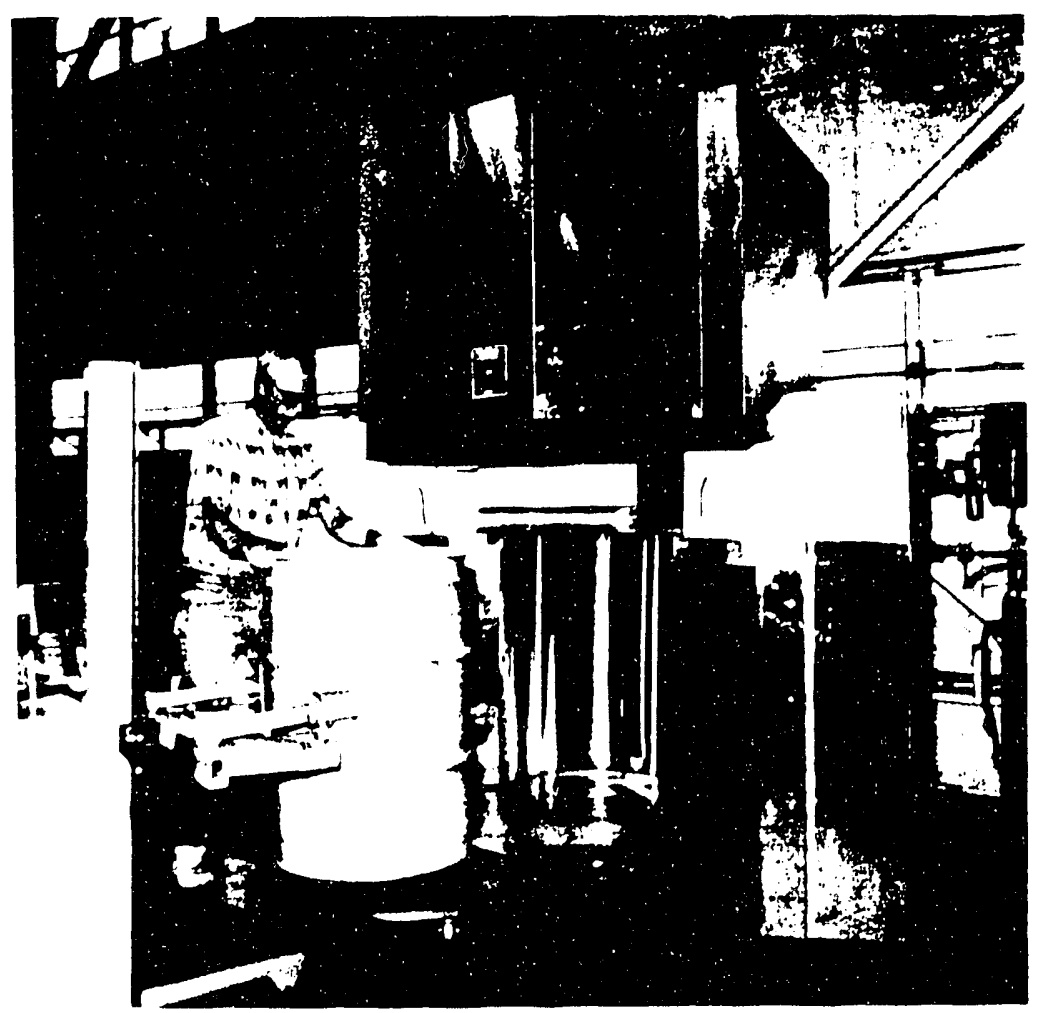


PHOTOGRAPHS AND FIGURES

(cont)

Fig. 3. The storage chamber of the shuffler is viewed from the front with the stainless-steel shroud removed to reveal the components of the drive mechanism.

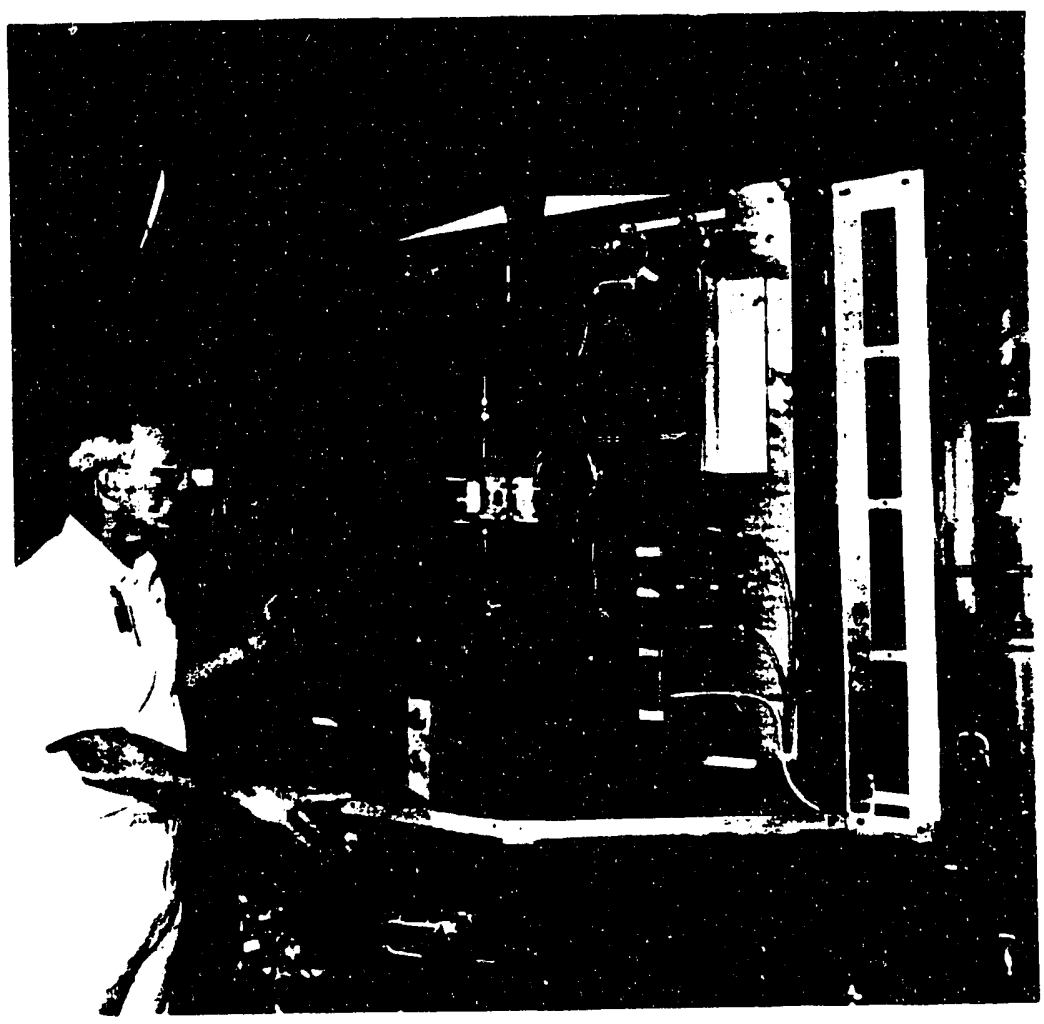

Figures 4-7 are sketches and a cross section of the shuffler from various perspectives. The main components are labeled.

The electronics rack is shown in Fig. 8 .

ACTION FLOW

When power is applied to the electronics rack, the Compumotor Indexer retrieves and runs a program already stored in its internal EEPROM if the Indexer has been set up properly. From the Indexer's main menu, push SETUP, then AUTORR; the AUTORCL and AUTORUN parameters should be set to YES. Return to the main menu by pressing the MENU button twice. Then press STORE to save this new setup and the START to run the Indexer's code. 


\section{ACTION FLOW}

(cont)

Front View

(Top Shroud Removed)

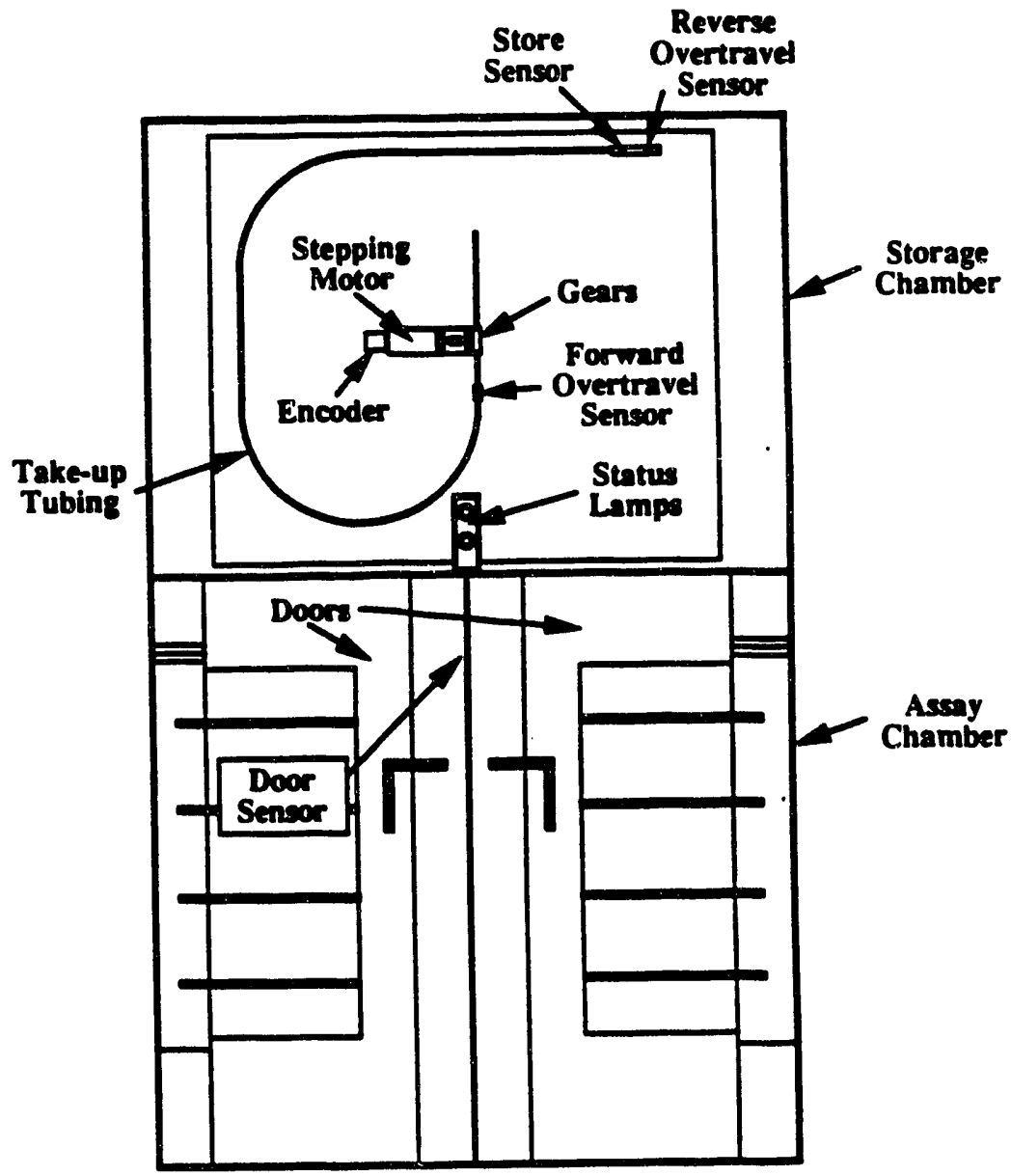

Fig. 4. The major components are identified on this sketch of the shuffler's sront with the doors closed.

Meanwhile the computer boots itself and automatically runs the shuffler code if this line is in the MS-DOS

AUTOEXEC.BAT file: "Ports.EXE" (or simply "Ports"); the quotation marks are not to be included.

A user communicates with the computer through the keyboard, the video monitor, a printer, and a bar code reader. 


\section{ACTION FLOW}

(cont)

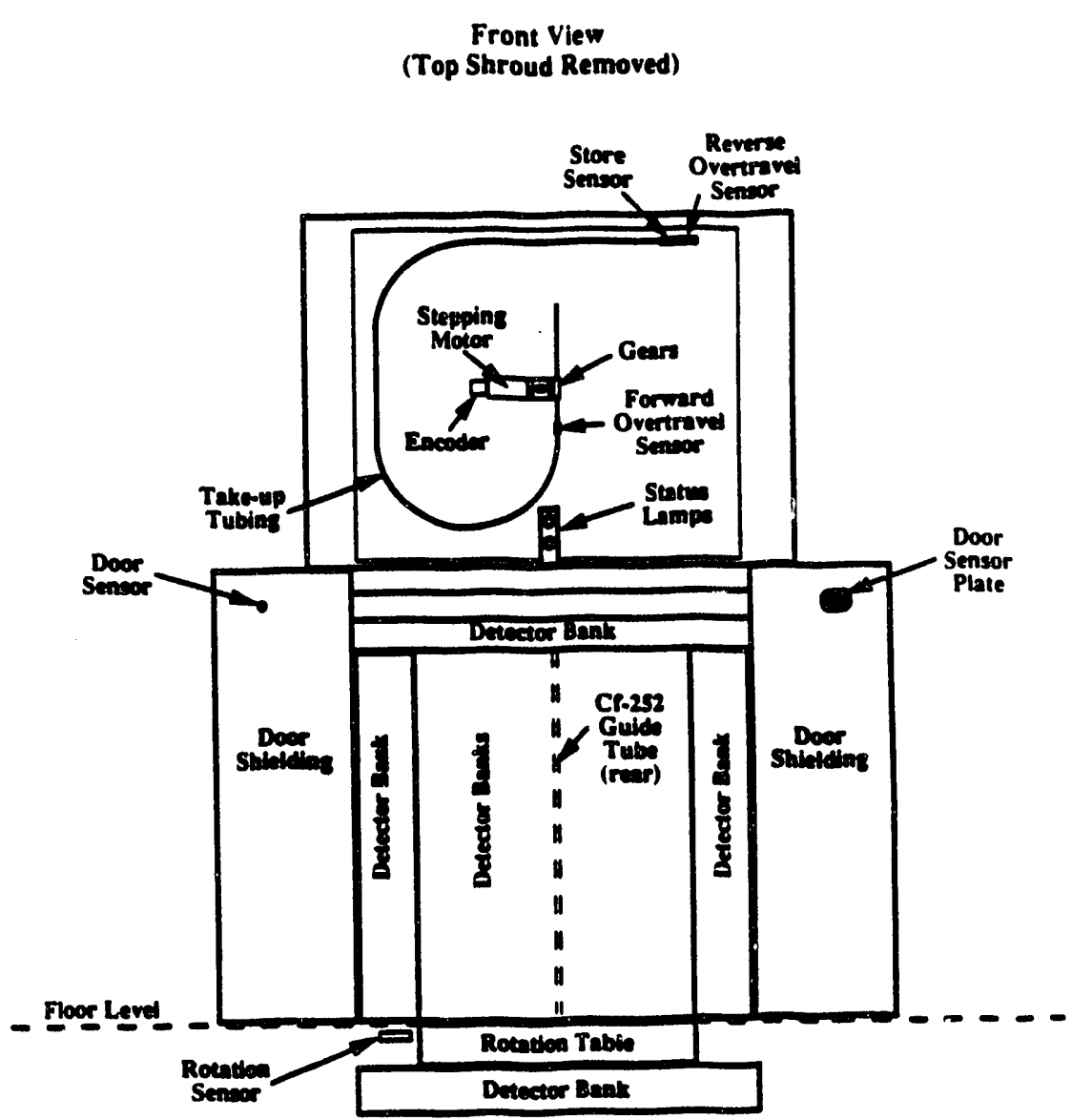

Fig. 5. The major components are identified on this sketch of the shuffler's front with the doors open. The components below the floor level are also indicated.

The computer communicates with the Compumotor Indexer and the Los Alamos scaler board through separate RS-232 serial lines. If necessary, the program for the Indexer may be downloaded into the Indexer over the serial line and then stored in the EEPROM. The printer is attached to another serial line.

The computer checks that communications with the Indexer, the scaler board, and the printer are functioning properly. If there is a problem, the user is so advised. 


\section{ACTION FLOW}

(cont)

Top View of Interior

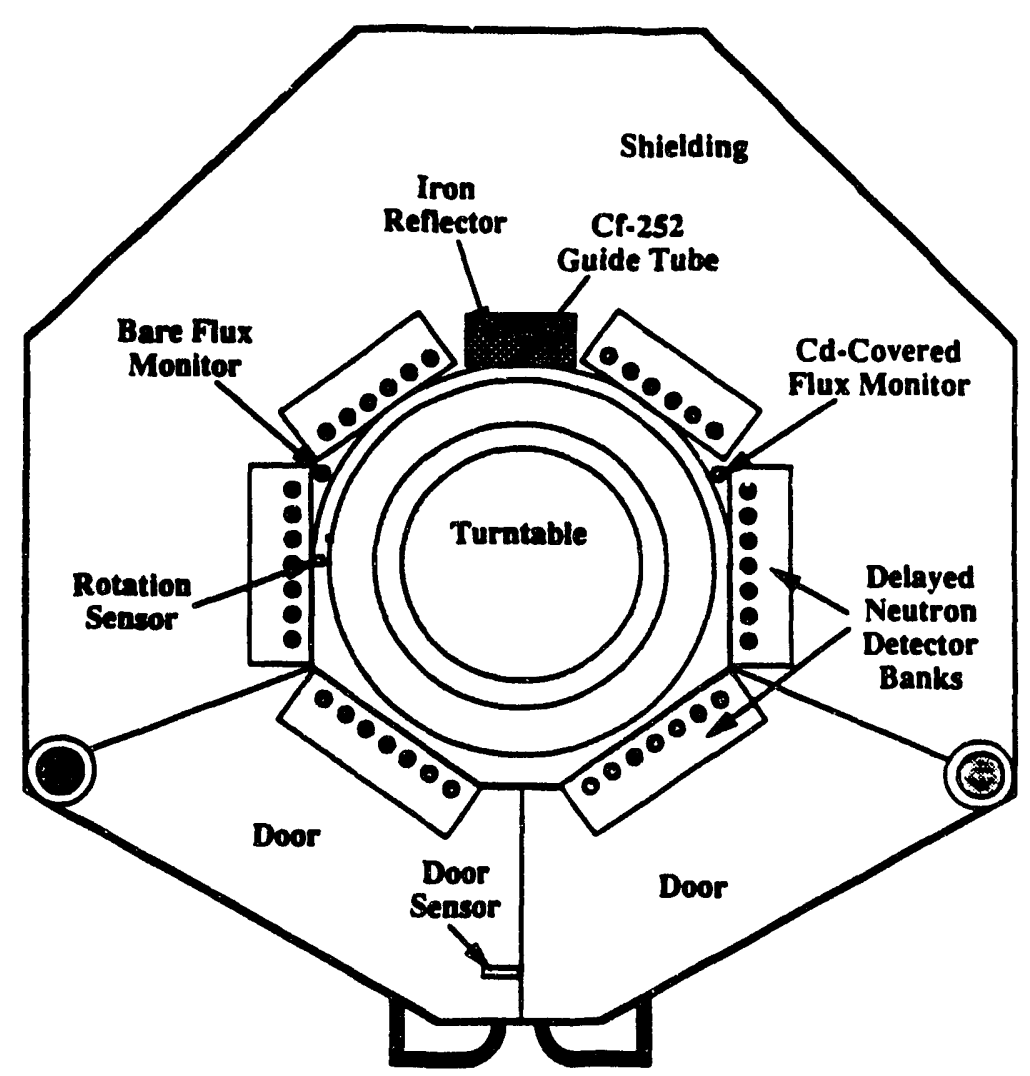

Fig. 6. The shuffler's assay chamber has been sectioned horizontally and is viewed from above. A drum rests on the turniable and rotates while the ${ }^{252} \mathrm{Cf}$ source irradiates it. After the source has been removed, the delayed-neutron detector banks count delayed neutrons. The flux monitors count neutrons from the ${ }^{252} \mathrm{Cf}$ source during the irradiation.

The user is asked for an identifier (name, company number, ...) and then a password; the password is not echoed to the screen. If this information is valid, the shuffler's computer notes the command level for the user (an operator, a supervisor, or a manager) and presents an appropriate menu of options. Only a manager has the option of creating or modifying valid user identifiers and passwords. 


\section{ACTION FLOW}

Side View

(cont)

Fig. 7. The shuffler is viewed from the side with the storage chamber's shroud in place. The gamma-ray ports have polyethylene plugs that may be removed to allow the measurement of activation gamma rays from the object being assayed.
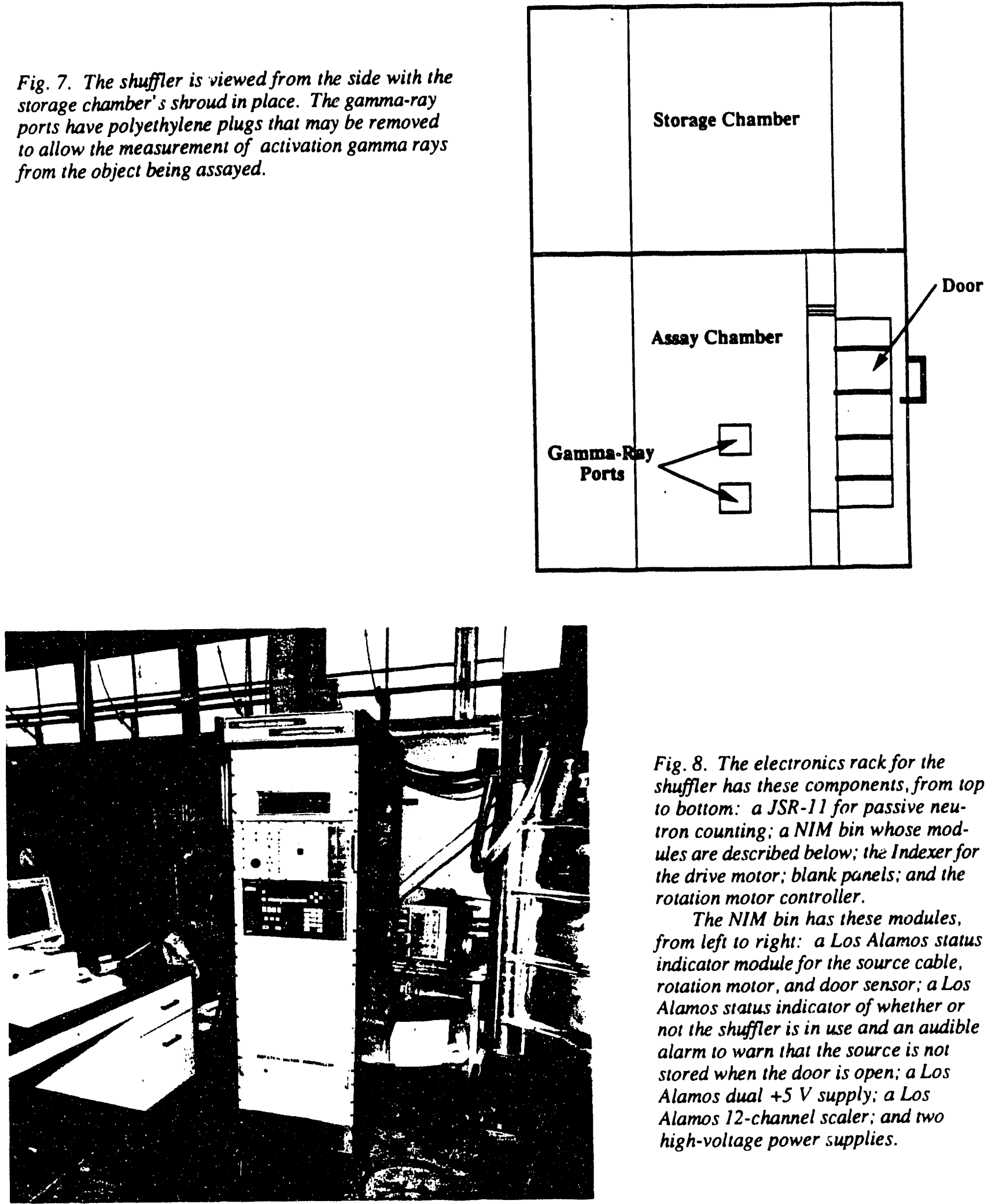

Fig. 8. The electronics rack for the shuffler has these components, from top to bottom: a JSR-11 for passive neutron counting: a NIM bin whose modules are described below; thi Indexer for the drive motor; blank panels; and the rotation motor controller.

The NIM bin has these modules, from left to right: a Los Alamos status indicator module for the source cable. rotation motor, and door sensor; a Los Alamos status indicator of whether or not the shuffler is in use and an audible alarm to warn that the source is not stored when the door is open; a Los Alamos dual +5V supply; a Los Alamos 12-channel scaler; and two high-voltage power supplies. 


\section{ACTION FLOW}

(cont)
The three command levels and their options are described in detail later in this manual. In general terms, there are options to perform assays, perform measurement control and safety checks on the shuffler, modify the operating parameters, review past data, and exercise diagnostic routines. 
INTRODUCTION

POWER CONDITIONER

NIM BIN

HIGH-VOLTAGE POWER

SUPPLY

Cable Connections

Switches and Dials
The proper settings of switches and dials for the electrical components are listed in this section. Most of the electronics is in the rack shown in Fig. 8. More details on the components and their interconnections are given in the companion Hardware Manual.

The settings recommended below are for normal operation. Deviations from these settings should be made only for good reasons and after careful consideration of the consequences. Some changes could make the shuffler inoperable while others could invalidate the current calibration.

The power conditioner at the bottom of the electronics cabinet has an ON/OFF switch that should be ON.

The ON/OFF switch of the NIM bin should be ON.

There are two identical high-voltage power supplies for the detector tubes.

Rear Panel:

The cable labeled HIGH VOLTAGE should be attached to the HIGH-VOLTAGE connector.

Nothing should be attached to the INHIBIT connector.

\section{Inside Chassis:}

The POLARITY switch should be set to POSITTVE.

\section{Front Panel:}

The LED on the front panel should indicate a POSITIVE polarity.

The high voltage for the detector tubes should be set at $1680 \mathrm{~V}$. To have this value, the KV switch should be set to 
Switches and Dials

(cont)
$1(1 \mathrm{kV})$ and the ten-turn potentiometer for $680(680 \mathrm{~V})$.

The potentiometer should be locked and not changed.

The ON/OFF switch should be set to ON. It is not necessary or even desirable to set the high voltage to zero before turning the power supply on or off.

Any change made in the high-voltage setting is likely to invalidate the calibration of the shuffler.

LOW-VOLTAGE POWER SUPPLY Theri are no internal adjustments in this module that provides $+5 \mathrm{~V}$ to the AmpTek amplifiers inside the junction box.

\section{Front Panel:}

The ON/OFF switch should be ON.

The LED should be ON.

There is a test point to confirm that the output voltage is indeed $+5 \mathrm{~V}$.

\section{Rear Panel:}

The cable labeled " $+5 \mathrm{~V}$ " should be attached to the connector.

ABC SWITCH

This switch routes one of the computer's RS-232 communication lines to either the shuffler's indexer or the JSR-11 shift-register counter in the electronics rack.

\section{Front Panel:}

The toggle switch should be set to SHUFFLER for shuffler operation. 
ABC SWITCH

(cont)

SHUFFLER STATUS LAMPS

Red Lamp
Rear Panel:

Three 9-pin D-connectors should be inserted into the sockets with the corresponding labels. From top to bottom these are "NCC" (neutron coincidence counter), "SHUFFLER," and "COMPUTER."

Two signals are prominently displayed by a status panel above the shuffler's doors and show the current status of the shuffler overall. The same lamps are indicated on a Los Alamos NIM that also contains an audible alert that the doors are open and the source may be exposed.

The red lamp is on when a measurement is in progress. This includes the passive background count that precedes the active interrogation using the ${ }^{252} \mathrm{Cf}$ source. Opening the door when the red lamp is on will abort any measurement in progress.

If for some reason it is felt necessary to open the door when this red lamp is on, independent verification should be made that the source is indeed stored. The store lamp on a NIM in the electronics rack will be on if the Compumotor Indexer is on and the source is stored. The LEDs on the scaler board NIM front panel should be flickering slowly from background; if they are on steadily, the source is not stored. Furthermore, if the end of the source's cable is visible above the store position proximity switch and the scaler LEDs are flickering slowly, the source is safely stored. To be sure, a gamma-ray meter can be held between the doors as the doors are slowly opened; if the meter reading increases, the source may be present and the doors should be closed.

When a user exits the shuffler code, both shuffler status lamps are turned off. 
Green Lamp

SCALER MODULE
When the shuffler code is running but a measurement is not in progress, the green lamp is on. This indicates that the door may be opened to load or unload a drum. During a measurement, the green lamp is off (and the red lamp is on). When a user exits the shuffler code, both shuffler status lamps are turned off.

This scaler has 12 channels. Channel 0 is used as a timer and is on continuously. Channels 1 through 8 count pulses from delayed-neutron detectors and flicker at a slow rate when the source is stored but are on steadily when the source is inside the assay chamber. Channels 9 and 10 count pulses from the flux monitors while the source is irradiating a drum. Channel 11 is not used.

\section{Front Panel:}

The lamp for channel zero should be on continuously. It is counting the internal clock's rate of $10 \mathrm{kHz}$.

A test point is available to check the internal $+5 \mathrm{~V}$ supply.

\section{Rear Panel:}

Of the 12 connectors, only those labeled channels 1 through 10 should be connected. They should be matched to cables labeled SCALER 1, SCALER 2, ..., SCALER 10; these are connected to delayed neutron detector banks 1 through 8 and then flux monitors 1 and 2, respectively.

Here are the physical locations of the delayed-neutron detector banks: left door (SCALER 1), left side (SCALER 2), left rear (SCALER 3), right rear (SCALER 4), right side (SCALER 5), right door (SCALER 6), top (SCALER 7), and bottom (SCALER 8). 
SCALER MODULE

(cont)
The left flux monitor is not covered by cadmium and corresponds to SCALER 9. The right flux monitor is covered with cadmium and corresponds to SCALER 10.

The output connector of SCALER 0 provides the sum of signals from channels 1-8 that then enters the JSR-11 for passive counting.

The 9-pin connector accepts the cable labeled SCALER from the computer. This is the serial communications link.

The ON/OFF switch is located on the front of the Indexer and should be on. The green status lamp should be $O N$.

A Los Alamos program must be running within the Indexer for the shuffler to operate. This program controls the stepping motor independently of the shuffler's computer. For example, the ${ }^{252} \mathrm{Cf}$ source is placed in its store position by the Indexer while the shuffler's computer waits for a signal from the Indexer that the procedure was completed successfully. If an operation is not completed within an expected time interval, the computer assumes the operation was unsuccessful and generates an error message.

The program to be run by the Indexer has been created outside the Indexer and is called "PORTS.PGM." It is downloaded into the Indexer by selecting the menu option "Diagnostics/Download Indexer." The user is prompted for the name of the code to be loaded into the Indexer, that name is "PORTS.PGM" for the code supplied by Los Alamos and should be given exactly as shown (without the quotation marks). 
Program
(cont)

COMPUTER AND MONITOR

PRINTER

AMPLIFIER/SCAS
The communication ports of the Indexer must be set correctly. From the SETUP option select RS232; the baud rate should be 9600 with 1 stop bit, 7 data bits, even parity, and DTE selected. If a change is needed to have these choices, be sure to press the ENTER button to have the changes used. From the SETUP option select REMOTE and set

TEXT to OFF; this is the correct setting for downloading and running the code.

The Indexer code can be stored in the Indexer's batterybacked memory with the SAVE option in the Indexer's menu.

The ON/OFF switch should be ON to both the computer and the monitor.

The printer's ON/OFF switch should be ON and the ONLINE lamp should be ON to indicate it is ready to receive signals. If the printer is off line, the computer will pause the first time it tries to write to the printer and present a message about the printer on the screen after waiting a few seconds.

A discriminator has been set on each AmpTek amplifier/SCA unit mounted in the junction box. These should never need adjusting; more details on them are given in the companion hardware manual. 
UNIFORM IRRADIATION
The purpose of this shuffler is to assay the masses of ${ }^{235} \mathrm{U}$ inside waste drums and other containers. This requires irradiating the drums throughout their volumes as uniformly as possible. If there are inhomogeneities in the ${ }^{235} \mathrm{U}$ distribution, the measured response will still give a correct assay value.

Uniform irradiation requires relatively high-energy neutrons. Thermal neutrons would not penetrate the dense materials within the drums, and the response would depend only on the nature of the material on the skin of the drum contents. (The mean-free-path length of thermal neutrons in 235U is only $0.03 \mathrm{~cm}$.) The neutrons from ${ }^{252} \mathrm{Cf}$ are high enough in energy to penetrate the matrix within each drum, and the materials of the assay chamber were chosen to make the fission probability as uniform as possible throughout the volume of each drum.

The assay chamber is lined with cadmium to prevent thermal neutrons from entering the chamber. Neutrons from ${ }^{252} \mathrm{Cf}$ or $235 \mathrm{U}$ could otherwise leave the chamber, become thermalized in the polyethylene around the detector tubes, re-enter the chamber, and (with high probability) cause fissions on the skin of the drum's contents. These thermal fissions would dominate and distort the assay process.

Uniform irradiation requires a sacrifice in the total fission rate for a given size of ${ }^{252} \mathrm{Cf}$ source; high-energy neutrons are less likely to cause fissions than are thermal neutrons. Fortunately the ${ }^{252} \mathrm{Cf}$ size could be increased to make up for the drop in fission probability and the source would still be fairly small. 
UNIFORM IRRADIATION (cont)
A potential problem with irradiation by high-energy neutrons is the production of delayed neutrons from ${ }^{238} \mathrm{U}$ instead of 235U. However, only a small fraction of the neutrons from $252 \mathrm{Cf}$ have enough energy to cause fissions in $238 \mathrm{U}$. Monte Carlo calculations indicate only about 1 fission in $238 \mathrm{U}$ for every 25 fissions in ${ }^{235} \mathrm{U}$ even if there is no moderating material within a drum. Most of the fissions in 238 $\mathrm{U}$ are stimulated by fast neutrons released after fissions of $235 \mathrm{U}$ rather than from ${ }^{252} \mathrm{Cf}$ neutrons directly; there is no way to eliminate this source of high-energy neutrons from inside the drums themselves, so the effects of $238 \mathrm{U}$ must be accounted for as part of the calibration of the drums. 
SAFETY FEATURES

POWER SWITCHES

COMPUTER BOOTING

Configuration Files
The earlier section called SAFETY FEATURES should be understood before startup. The modest hazards are easily avoided when understood.

All power switches may always be left on. If power to the electronics needs to be turned off, a single switch controlling power to all the equipment may be turned off.

The motor driving the ${ }^{252} \mathrm{Cf}$ source may be turned by hand if the power to the motor is off. Power to the motor alone can be removed by unplugging the power cord entering the power/signal box on the side of the computer. Compumotor recommends that the power connector entering the power driver not be disconnected because the conductors may be damaged; the driver is the black box with cooling fins mounted on the front of the storage chamber inside the stainless-steel shmud.

At this point, power to all other components of the shuffler should have also come on. If the NIM bin power is not on, the scaler NIM will not be able to respond to an inquiry from the computer and will lead to an error message when an attempt is made to communicate with the scaler.

When power is applied, the various lamps on the front panels of the different NIMs should have the ON-OFF patterns described earlier.

Turning the power on to the computer initiates the booting sequence if the AUTOEXEC.BAT file has the line "Ports.EXE" that automatically starts the shuffler code.

The same code is used on both the X-326 and X-744G shufflers, but there is one hardware difference that is reflected in what are called the "configuration" files read by 
Configuration Files (cont) the code. The stepping motor of the X-326 shuffler takes 500 encoder steps for an inch of ${ }^{252} \mathrm{Cf}$ travel, while the servo motor of the X-744G shuffler takes 625 steps for an inch of travel. The proper configuration file for a shuffler must be present for the code to read.

The name of the configuration file is PORTS2.CFG for both shufflers. There are seven lines in the configuration file. It is an ASCII file and can be edited by any ASCII editor. Its contents can be checked simply with the IBM-DOS commands TYPE PORTS2.CFG or PRINT PORTS2.CFG. Each line has information for the shuffler code plus comments explaining the significance of the line. Here is a typical listing for the X-744G shuffler (shuffler number 2 at Portsmouth):
PORTSMOUTH SHUFFLER

1.0

November 25, 1991

No. 2

$\mathrm{Y}$

8.5

625.0

$$
\begin{array}{r}
l^{*} \text { program name */ } \\
l^{*} \text { version number */ } \\
/ * \text { release date */ } \\
l^{*} \text { instrument id */ }
\end{array}
$$

/* rotation table installed: $\mathrm{Y}$ or $\mathrm{N} * /$

* rotation table delay time *I

/* steps-to-inches conversion */
The last line has the number of steps per inch, which differs between the two shufflers. The instrument ID will be No.1 for the X-326 shuffler and the rotation table delay time may differ slightly between the two shufflers.

To stop the rotation turntable with the proper orientation, a bolt on the table triggers a proximity switch and then a certain wait time is needed to achieve that orientation. After that delay time the power to the table's motor is cut. 
SIGN ON

MENUS AND FORMS

Main Menus
After the shuffler code executes, the user is asked for an identifier (name, company number, . . . ) and then a password. Both of these items are "case sensitive," meaning the NIM bincapitalization is important. For example, "sharon" and "Sharon" are different words and not interchangeable. As a user enters a password, it is not echoed to the screen. If a mistake is made in either item, the user is returned to MS-DOS and may try again by executing the code by typing "Ports."

A manager must maintain the list of valid identifiers and passwords and establish which are operators, which are supervisors, and which are managers. When someone logs on, a set of menu options will be made available that are appropriate to that user (most limited for operators, complete availability for managers, and complete availability except for identifier/password access and control for supervisors).

The next screen generated by the shuffler code shows the date and time, which can be changed if they are incorrect. It is very important that at least the date be correct because it is used to correct for the decay of the ${ }^{252} \mathrm{Cf}$ source.

The format of the date is " $\mathrm{mm} / \mathrm{dd} / \mathrm{yy}$." An example is 11/12/90 for November 12, 1990.

The format of the time is "hh:mm:ss." Examples are 10:33, 10:33:00, 10:33:27, and 09:22 (but not 9:22).

One of three main menus will appear on the screen, corre sponding to the access level previously assigned by the manager for the person who has signed on. Operators, supervisors, and managers have different menus. 
Main Menus

(cont)

The main menu of options is displayed across the top of the screen. A message line on the bottom of the screen shows the keys that can be pressed to move through the menu and select suboptions. Different suboptions drop down as the highlight is moved with the horizontal arrow keys. A suboption within an option is highlighted with the vertical arrow keys.

In general, moving through menus, lists, and forms is done with arrow keys and the Return or TAB keys.

The F10 key is used to accept the information on the screen. If a menu option is highlighted, the F10 key indicates to perform that option. If a list is displayed, the F10 key indicates that the item in the list that is highlighted is to be selected. If a form is displayed, the F10 key indicates that the entries in the form are to be used.

Forms

Some suboptions lead to forms that require information from the user. The bottom line of the display indicates how to move through the form, how to accept the current values in the form, and how to exit the form. Pressing the Return key or TAB key steps through the items in the form. The vertical arrows cause the cursor to move vertically. The values shown in the form are accepted with the F10 key.

The supervisor's and manager's form, following the selection of Archives from the main menu, has three items. There are several possibilities for each item; the user may press the F9 key to get a list of the possibilities and not have to remember how they are spelled. The desired entry is selected from the list, not typed in from the keyboard. The bottom status line on the screen reminds the user of this procedure. 


\section{Forms}

(cont)

Lists

POWER DOWN
If entries in a form have been made but the user wishes to exit the form without these changes going into effect, the ESC key can be pushed. The user will then be asked to confirm that any changes made to the form are indeed to be ignored.

When a list is presented, the vertical arrow keys allow scrolling through the list. The F10 key causes selection of the highlighted member of the list.

There is one "list" that differs in character from the description above. When a list of archived data is displayed on the screen there are no choices to be made except to scroll through a list longer than the screen can show. To exit this list, the ESC key must be pushed. The status line on the bottom of the screen again prompts the user of these actions.

The only action needed to power down the computer is to flip the ON/OFF switch to OFF. The monitor and printer have individual power switches. 
GENERAL

MEASUREMENTS

Assay
An operator can perform several types of measurements, do safety checks, and log off the computer. These are now described in detail.

The purpose of the shuffier is to assay waste drums for their 235U contents, but other measurements are pmvided to check the operation of the shuffler and to help calibrate it.

The purpose of this option is to perform an assay on a drum. The computer has a dialog with the operator before the measurement beisins.

The shuffler doe, not know if the drum is acturdly present in the assay chamber, so it asks the operator if the dum is inside. If the answer is "no," the assay is abcrted and the main menu returns.

The second request is for the drum's ID. This can be entered through the keyboand or the bar code reader. The first two characters are used by the code to select one of several possible assay parameters. A set of parameters can be created (by a supervisor or manager) appropriate to the size and matrix material of a type of drum; a different calibration is needed for each type. If the user enters $n$ ID whose first two characters do not correspond to a known drum type, the operator is 30 informed and the option menu reappears.

The assay measurement will begin immediately if the proximity switch on the doors indicates that the doors are closet. If the doors are open, the operator will be reminded to close them and the user can continue the assay after closing the dcors. 
Assay

(cont)
The display on the monitor tracks the progress of the assay. A background count is first taken, followed by the shuffling of the ${ }^{252} \mathrm{Cf}$ source to alternately irradiate the drum and count delayed neutrons. The status lamps mounted on the shuffler's body also track the assay in a gross way; the green status lamp is off and the red lamp is on during measurements.

When the assay is finished and the result acknowledged by the user, the option menu reappears and the green status lamp should be the only lamp on.

A Standard Check is a combination of two assays whose expected results are well established. The operator can do this check at any time, but if the time interval since the last check exceeds a value stored in the computer, the computer will refuse to do any measurement other than a standard check. If for some reason it is necessary to do some other measurement before the standard check requirement is satisfied, any supervisor or manager has the option to override the requirement.

The first assay is done with an empty assay chamber (no drum present). This is to confirm that the assay chamber is free of fissile material that would lead to an incorrect assay of a drum. The result is expected to fall within a range (near zero) placed in a parameter file by a supervisor or manager.

The second assay in a Standard Check is with a standard drum whose assay result has been well established. The result of this assay is compared to a reference value (also stored in a parameter file) and is expected to statistically agree with the reference value at a specified confidence level. Mathematical details are presented in a later section (STATISTICAL CHECKS). 
Standard Check

(cont)

Bias and Precision Checks

Precision Check

Calibration Data
If either of these assays is outside its acceptable range, the Standard Check has been failed. The operator must repeat the entire check. If a second failure occurs, the operator is told that a supervisor or manager is needed and the operator is automatically logged off the computer.

These checks are made by a series of repeated assays on a drum of known 235U mass. (This could be the same drum used in the Standard Check, but need not be.) The number of assays taken is set in the parameter file by a supervisor or manager.

When the series of assays is finished, the average result and the standard deviation are compared to expected values (within specified confidence levels) stored in the parameter file. Details on this are in the STATISTICAL CHECKS section.

The Precision Check is similar to the Bias and Precision Check just described. But in this case there is no expected assay result for the drum and the $235 \mathrm{U}$ content of the drum need not be known.

The average assay value and the standard deviation of the series of assays are displayed for the user. This check is neither passed nor failed.

The assay data analysis requires calibration curves based on measured count rates from special drums whose $235 \mathrm{U}$ contents and enrichments have been previously determined.

These calibration drums can be assayed under this suboption and the data analysis of the count rates will end with corrected count rates rather than $235 U$ masses. With the known 


\section{Calibration Data}

(cont)

Plant Communications masses and newly measured count rates, new calibration curves can be constructed.

A supervisor or manager should make sure that the flux monitors are properly used while taking calibration data. The matrix inside the standard drums will probably be known and NO flux monitor correction should be applied; this is set in the Assay Diagnostic section of the parameter file for this type of drum.

At the end of a measurement, the shuffler's computer may attempt to transmit the following list of information to the process computer. Whether or not an attempt is made is controlled by a parameter set by a manager.

\section{Drum ID}

\section{Current Date}

Current Time

Operator ID

Name of current parameter file

Date of current parameter file creation or modification Time of current parameter file creation or modification Raw background counts in delayed neutron bank \#1 Raw background counts in delayed neutron bank \#2 Raw background counts in delayed neutron bank \#3 Raw background counts in delayed neutron bank \#4 Raw background counts in delayed neutron bank \#5 Raw background counts in delayed neutron bank \#6 Raw background counts in delayed neutron bank \#7 Raw background counts in delayed neutron bank \#8 Raw background counts in flux monitor \#1

Raw background counts in flux monitor \#2 Background count time

Raw assay counts in delayed neutron bank \#1

Raw assay counts in delayed neutron bank \#2 
Plant Communications

(cont)
ASCII Data File
Raw assay counts in delayed neutron bank \#3

Raw assay counts in delayed neutron bank \#4

Raw assay counts in delayed neutron bank \#5

Raw assay counts in delayed neutron bank \#6

Raw assay counts in delayed neutron bank \#7

Raw assay counts in delayed neutron bank \#8

Raw assay counts in flux monitor \#1

Raw assay counts in flux monitor \#2

Total count time for delayed neutrons

Total count time for flux monitors

Type of measurement: assay; standard check; bias \& precision check; precision; calibration

Corrected count rate

Grams of 235U (zero if no calibration curve applied)

Uncertainty in the assay value

Error number

A Standard Check will generate two sets of results, one for the empty chamber and another for the standard drum. Each set will give the information in the above list. Each assay in the set of assays of a Bias and Precision Check or a Precision Check will also generate the information in the above list.

A special data file called "ASCII.DAT" can be added to after every measurement. A manager can manipulate this file more easily than the binary file used by the data base. These data can be read by an ordinary editor, and a supplementary code called "DataPrep.EXE" can read the file, print the file, perform analyses, or make files of selected data suitable for spreadsheet and plotting programs.

This data file should regularly be copied to a disk using a meaningful name. If a copy is made each day, that day's date could be used for the name: data from April 30, 1991, 
ASCII Data File

(cont) would have a name "043091.DAT," for example. After copying the file, it should be deleted using the MS-DOS command: "del ASCII.DAT."

If the data file is not deleted regularly, it will continue to grow in size. Disk space will become wasted and editors will eventually be unable to open and read it.

The data format used in "ASCII.DAT" is this:

Type of measurement (alphanumeric characters)

Item ID (alphanumeric characters)

Date and Time (mm/dd/yy hh:mm:ss)

Operator's ID (alphanumeric characters)

Enrichment percentage (number)

Background counts of channels 1 through 10 , one per line (numbers)

Background count time (number)

For each shuffle,...

Forward transfer time (number)

Irradiation time (number)

Reverse transfer time (number)

Count time (number)

Counts of channels 1 through 10, one per line (number), channels 1 through 8 are for delayed neutrons, channels $\mathbf{9}$ and $\mathbf{1 0}$ are for flux monitors Blank line to separate measurement data

This check is not required of a user by the shuffler's computer at any particular time. The equipment involved has been reliable throughout extensive testing and use, but it is in the best interest of all users to perform the check on a regular interval. The safety check has several steps to test important hardware features; the steps are now described in the order they are taken. If any of these checks fail, they must be 
SAFETY CHECK

(cont)

\section{Door Interlock}

${ }^{252} \mathrm{Cr}$ Proximity Switches repeated. If there is a second failure, the operator is forced to seek the help of a manager or supervisor.

The computer checks that the ${ }^{252} \mathrm{Cf}$ source is stored. If it is not, the Compumotor Indexer is commanded to store it. The operator is asked to open the shuffler's doors and to tell the computer when the doors are open. The shuffler's computer then checks that the doors' proximity switch agrees that the doors are open.

The operator is asked to close the shuffler's doors and to tell the computer when the doors are closed. The shuffler's computer then checks that the doors' proximity switch agrees that the doors are closed.

The shuffler's computer checks that the doors are closed. If they are not, the operator is asked to close the doors. When the proximity switch indicates that the doors are closed, the check of the ${ }^{252} \mathrm{Cf}$ source's proximity switches begins.

The ${ }^{252} \mathrm{Cf}$ source is moved into the assay chamber to clear both the store and the forward overtravel proximity switches; the distance taken is set by a supervisor or manager in the parameter file. The source is then returned to the store position.

The source is then moved onto the reverse overtravel switch and finally returned to the store position again.

The computer turns off both lamps and asks the operator to confirm that they are in fact off.

The computer turns on the red status lamp and turns off the green status lamp. The operator is asked to confirm that the red lamp is on and that the green is off. 
Status Lamps

(cont)

\section{DIAGNOSTICS}

Test Door Proximity Switch

Test Californium Proximity Switches

Test Status Lamps

Store Californium
The computer turns on the green status lamp and turns off the red status lamp. The operator is asked to confirm that the green lamp is on and that the red is off.

These options are designed to exercise specific portions of the shuffler to aid in checking their operations without the additional complexities of a measurement. The Indexer's code can also be downloaded into the Indexer through a Diagnostics option.

The doors' proximity switch can be tested by selecting this suboption and following the prompts given on the monitor. This test is identical to the Door Interlock check previously described under the Operator's Safety Check.

The store and two overtravel proximity switches are tested for their responses to the free end of the $252 \mathrm{Cf}$ source's cable. This test is identical to the ${ }^{252}$ Cf Proximity Switches' check previously described under the Operator's Safety Check.

The two status lamps can be exercised for proper operation through this suboption. This test is identical to the Status Lamps' check previously described under the Operator's Safety Check.

Selecting this suboption causes the ${ }^{252} \mathrm{Cf}$ source to return to its store position. Even if the source is already stored when this suboption is selected, the source will be moved a short distance and ihen stored again.

The Store Californium suboption is a convenient way to return the source to the store position from an arbitrary position; it does not require the user to know the distance from the store position. 
Move Californium
The ${ }^{252} \mathrm{Cf}$ source can be moved an arbitrary distance with this suboption (to positions between store and forwandovertravel locations). The distance is always from the source's current position and is given in encoder steps for the shuffler in Building X-326 where there are 500 encoder steps per inch of travel, or in motor steps for the shuffler in Building X-744G where there are 625 steps per inch. A positive number of steps are taken toward the assay chamber away from the stored position; a negative number of steps are taken away from the assay chamber toward the stored position.

If the specification for the Building X-326 shuffler is to take 5000 steps with 500 steps meaning $1 \mathrm{in}$. of travel, the source moves 10 in. toward the assay chamber (assuming the forward overtravel switch is not uncovered by the cable); if it is next specified to take 1000 steps, the source moves another 2 in. toward the assay chamber; if it is then specified to take -2500 steps, the source is pulled 5 in. away from the assay chamber, the net distance moved is $+10+2-5=7$ in. in the direction of the assay chamber.

The acceleration (motor steps $/ \mathrm{s}^{2}$ ) and velocity (motor steps/s) can also be specified for the move. The Indexer requires that these be given in units of motor steps, even if encoder steps are used to specify the distance to be moved. There are $\mathbf{2 5} 000$ motor steps per revolution for the stepping motor on the Building X-326 shuffler and 5000 motor steps per revolution for the servomotor on the Building X-744G shuffler. Fast motions typically use accelerations and velocities in the millions; slow motions typically use accelerations and velocities in the tens of thousands. If an acceleration or velocity is requested that is beyond the limit imposed by Compumotor, the Indexer automatically rounds the value down to the limit and proceeds with the motion. 
Move Callfornium to Test

Position
Count with No Californium Motion
Selecting this suboption initiates a series of steps. This provides a simple check on the electronics by comparing the count rates in the detectors with historical values.

The user is asked what count time (in seconds) is to be used. A background count of that duration is then taken, after making sure the source is in its store position and will not contribute to the count.

The source is next moved toward the assay chamber by the distance specified in a parameter file (the standard test position) and a second count of the same duration is taken. The source is then stored.

The background count rate is subtracted from the second count rate and a correction factor for the decay of the ${ }^{252} \mathrm{Cf}$ source applied.

A report of these numbers is given to the user through the video monitor and the printer.

When this suboption is selected, the supervisor is asked for the duration of the count (in seconds). The count is then started regardless of the position of the ${ }^{252} \mathrm{Cf}$ source or the doors' positions. (Radiation safety requires that the source be stored when the doors are open. As long as the stepping motor Indexer is on and running its code, the source will always be stored automatically when the doors are not completely closed.)

At the end of the count time, the monitor shows the readings of the scaler board's 11 channels ( 0 through 10$)$. Channel zero's displayed value is the count time in seconds. 


\section{Full Printout}

Print Last Results

Test Rotation Motor

Set Parallel
A measurement done after this suboption has been selected will generate a printout on the printer showing the raw data just gathered and all the intermediate values in the data analysis. Every step can be checked for the expected effect.

This suboption stays in effect until a second selection of this suboption turns the feature off. A message box on the monitor informs the supervisor whether the feature has just been turned on or off.

When this suboption is selected, the data in the computer's memory from an assay are reanalyzed and the results sent to the printer.

If Full Printout is ON, the full set of data and data analysis are printed. Otherwise, only two lines summarizing the assay are printed.

The rotation motor for the turntable can be toggled $O N$ and OFF by selecting this suboption.

Each bit of the parallel output port in the Indexer can be toggled individually after selecting this suboption. (The signal from the computer to toggle a bit is sent over the serial link between the computer and the Indexer.) The bits control these hardware items:

Bit 1 Red lamp, Machine ON

Bit 2 Audible alarm

Bit 3 Rotation motor

Bit 4 Green lamp, Machine OFF

Bit 5 No effect

Bit 6 Both lamps and the rotation motor

Bit 7 Both lamps, the rotation motor, and the alarm 
Set Parallel

(cont)

Watch Parallel

Download indexer

Write ASCII data

EXIT
The signal from the computer to toggle a bit is sent over the serial lint: ietween the computer and the Indexer.

The bits of the parallel input port in the Indexer can be examined individually after selecting this suboption.

The bits correspond to these hardware items:

Bit 1 Forward overtravel sensor

Bit 2 Store sensor

Bit 3 Reverse overtravel sensor

Bit 4 Door sensor

Bit 5 Control rotation motor

The code for the Indexer can be taken from a computer file and downloaded into the Indexer. The user is asked for the name of the file; the file supplied by Los Alamos National Laboratory is named "Ports1.PGM" for the X-326 shuffler and "Ports2.PGM" for the X-744G shuffler.

An ASCII file of "raw" data can be written after every measurement. This suboption toggles that activity on and off.

After selecting this option, the operator is asked to confirm that the intent is truly to exit the code. If the answer is "no," the main menu options reappear. If the answer is "yes," he is logged off. 
GENERAL

MEASUREMENTS

SAFETY CHECK

ARCHIVES
The supervisor has all the options of an operator, plus the ability to perform measurements even though it is time for a Standard Check or a check has failed, plus the ability to alter parameters that affect the operation of the shuffler.

This is identical to the Measurements' option for an operator, but an assay may be taken even though it is time for a Standard Check or a Standard Check has just been failed. If a supervisor elects to do a measurement under one of these unusual conditions, he or she is reminded that a check has not been passed and asked if he or she wishes to continue with the measurement. If the response is "no," he or she is returned to the supervisor's menu; otherwise, the measurement is performed.

This Safety Check is identical to the operator's in every way except that if it is failed twice the supervisor can still exercise all the menu options.

Results of previous measurements (of all types) are saved in an archive file for later review. The number of entries in this file is given by a parameter in the General Parameter file under the subheading Archiving and Communication Parameters.

Each archival entry has the list of information given earlier in the section OPERATOR'S OPTIONS/Measurements/Plant Communications. In addition each entry shows whether or not the data were successfully transmitted to the plant's computer.

When this file is full and a new measurement has been completed, the oldest measurement result is erased from the file to make room for the newest, unless the next oldest result has not been written to the process computer. 


\section{ARCHIVES \\ (cont)}

Log Search Type

Search Mode
When the archive file is full and communication with the plant's computer has been active, the next assay's results will be stored and the oldest data removed from the file.

When the archive file is full and communication with the plant's computer has been inactive, another assay cannot be taken. Data not yet sent to the plant's computer cannot be removed from the archive file (and thus lost), so a new assay is not allowed.

To take new measurements with an archive file full of results not transmitted to the plant's computer, the archive file must be emptied. This is done with a data base utility called "IDA." This is run from MS-DOS. After typing "IDA," select "OPEN" with the horizontal cursor keys and push RETURN. The only file name likely to be displayed is "dB_UMON," so accept that file name by pushing RETURN. Next select "INITIALIZE" and push RETURN; a warning will be given that all data in the file will be lost, but this is the intent so type " $y$ " for "yes" and push RETURN. Select "QUIT" and push RETURN to leave "IDA."

When the technical supervisor selects the Archives option, he or she can select to review all the data, only assay results, only standard checks (either empty or with standard drum), only bias and precision checks, only precision checks, only calibration data, only data not yet transmitted to the process computer, or only measurements that were completed with an internal malfunction detected.

Once the search type has been selected, the amount of data of that type to be reviewed can be restricted in two ways. A number of the most recent results can be requested, or beginning and ending dates and times can be specified. 
Search Mode

(cont)

Display Device
If beginning and e $\mathrm{ig}$ clates and times are to be used, the supervisor is prompted for the beginning date (dd-mm-yy format), the beginning time (hh:mm), and then the ending date and time.

The data can be sent to th : video screen, the printer, both the screen and the printer, or a disk file. If a disk tile is selected, the ASCII file named 'OUTPUT.DAT' is created.

To return to the main menu, push the F10 or ESC key.

A large number of parameters are used to specify the operation of the shuffler. A few features are inflexible (for example, the number of mc cor steps per inch) and a few others are in the Compumotor Indexer's code that can only be changed with some reprogramming in the Indexer's language (for example, the details of the process called by the Store Californium Diagnostic suboption).

The manager first seiects one of these actions to take with some parameters: Edit, Print, List the Change History, or Print the Change History. The Change History is a collection of notes entered by managers after changing parameters; if nothing was entered, that note is blank.

There is a set of general parameters that are independent of any specific drum (aside from the standard drum) that may be assayed. There is another set of parameters for each type of drum that a supervisor or manager wishes to define. The maximum number of drum types allowed with the software delivered with the shufflers is 18 .

General

A manager who selects to edit the General Parameters suboption next chooses from a list of categories of parameters. Each is described below. 
General
(cont)
Archiving Parameters. Maximum Number of Measurement Results to Archive Locally. A database in the shuffler's computer will retain the number of measurements given here, assuming the memory limitations of the computer are not exceeded. The main purpose is to store data while the EtherNet connection is down and data cannot be transmitted to the plant computer. This number can range from 1 to 9999 ; 1000 is recommended.

Send Results to Plant Computer [Y/N]. Any attempt to send measurement results to the plant's process computer is controlled by this parameter. If the choice is not to make the attempt, the shuffler can be operated as a stand-alone instrument. The results will be archived on the shuffler computer's hard disk and will be transmitted automatically to the process computer if the communication link is ever activated. This parameter is either " $Y$ " for yes or " $N$ " for no.

If the communication link does not exist, this parameter should be set to "N." Otherwise there will be delays and error messages that complicate the operation of the shuffler.

Bias and Precision Parameters. Number of Bias/Precision Assays. A number of repeat assays will be made on a standard drum that has an established assay value. That number is specified here. The range is from 2 to 9999 . The statistical check is better with a large number of assays, but the time allowed for the check may cause a small number to be used. Three may be sufficient but five is better and fifteen is better yet.

Time Delay Between Assays (sec). After an assay, the drum continues emitting delayed neutrons for a few minutes. The 
General

(cont) next assay will begin with a background count, so it is necessary to wait for the emission of these delayed neutrons to essentially cease. The range of the delay is 0 to $9999 \mathrm{~s}$. A time of $240 \mathrm{~s}$ is recommended; shorter times are likely to cause incorrect backgrounds and assays.

Expected Average Assay Result. The average of the repeated assay results is expected to have this value. It must be determined from previous experience with the drum. The range of values is 0 to $9999.9999 \mathrm{~g}$. The value depends on the item selected for use in this check.

Confidence Level Percentage for Detecting Bias Failure. This parameter is the probability of not declaring that an assay differs significantly from the expected value when indeed that is the case. This is written as $100(1-\alpha / 2) \%$, where $1000 \%$ is the probability of declaring a difference when in fact no difference exists. The range of values is $50 \%$ to $99.99 \%$, with $95 \%$ recommended.

Expected Standard Deviation of Assay Results. The standand deviation of the repeated assay results is expected to have this value. This value is determine by many previous assays. The range of values is 0 to $9999.9999 \mathrm{~g}$, but the actual value must be determined from experience with the drum selected for use in this check.

\section{Confidence Level Percentage for Detecting Precision}

Failure. This parameter is the probability of not declaring that an assay differs significantly from the expected value when indeed that is the case. This is $100(1-\alpha) \%$, where $100 \alpha \%$ is the probability of declaring a difference when in fact no difference exists. The range of possible values is $50 \%$ to $99.99 \%$, with $95 \%$ recommended. 
General

(cont)
Nuclear Constants. The nuclear constants given here are standard values determined and used by nuclear physicists for decades. The delayed neutron constants are given in detail in the Los Alamos National Laboratory report LA-12105 (May 1991) by P. M. Rinard, "Shuffler Instruments for the Nondestructive Assay of Fissile Materials."

${ }^{252}$ Cf Decay Constant (per day). The decay constant of the source in units of reciprocal days is needed to normalize the measured count rates to a reference date. The decay constant is $\ln (2)$ divided by the half life. The current accepted value is $0.0007172 d^{-1}$; this corresponds to a half life of $2.646 \mathrm{y}$.

2.5 Cf Decay Reference Date. Count rates will be normalized to this date to make it seem that the source strength does not decay with time. The calibration curves should be based on this same date. If this date is changed, old calibration curves are invalid and new ones should be developed. It is not necessary that this date be the date when the last calibration was made, although that is a reasonable choice. The format of the date is dd-mm-yy.

$235 U$ Precursor Group Fractional Yields. The delayed neutrons from ${ }^{235} U$ are described by the production of six groups of precursor nuclides. The fractions of the total precursor yield that fall into each group are given here and are unlikely to ever need changing.

The recommended yields, starting with group 1, are $0.000210,0.001420,0.001280,0.002570,0.000750$, 0.000270 .

235U Precursor Decay Constants. The decay of each precursor group is taken to be exponential and the six decay 


\section{General}

(cont) constants are specified here and are unlikely to ever need changing.

The recommended decay constants, starting with group 1 , are $0.012440,0.030510,0.111400,0.301400,1.136000$, $3.014000 \mathrm{~s}^{-1}$.

Item Type Parameters. Number of Types. An item in this shuffler is a drum. The number of drum types that might be measured is specified here. The number cannot be greater than 18.

Names of Drum Types. The names of the drum types are given here. The first two characters are compared with the first two characters in a drum's ID, which are expected to specify the drum type.

\section{Standard Check Parameters. Maximum Time Between} Checks. If the time interval since the last Standard Check exceeds this value, an operator is forced to do a Standard Check before doing any other measurement. This interval can range from 0 to 999.99 days, according to the plant operating requirements.

Empty Chamber Expected Assay Value. This is the value expected from the first part of the Standard Check where the assay chamber is empty. The range is from -999.9999 to $9999.9999 \mathrm{~g}$; a value of $0.0000 \mathrm{~g}$ is recommended unless holdup material is known to be present.

Acceptable Deviation from Empty Chamber Assay. If the empty-chamber assay value differs from the expected assay value by more than this amount, the checix has failed. Otherwise the check passed. The value can range from -999.9999 to $9999.9999 \mathrm{~g}$. 
General

(cont)
Assay Value of Standard Item. This is the value expected from a standard drum using the set of parameters appropriate to that type of drum. The range of values is from $\mathbf{0}$ to $9999.9999 \mathrm{~g}$. The value that should be used depends on the item selected as the standard.

Confidence Level Percentage for Detecting Failure. The measured and expected assay values for the standard drum will differ. This parameter sets the probability that the difference is statistically consistent with a true difference of zero. The range of probabilities is $50 \%$ to $99.99 \%$; $95 \%$ is recommended.

Test Position Parameters. Standard Californium Test Position (Inches from Store). This is the distance in inches to be moved from the store position toward the assay chamber. This distance is used in the "Move Californium Source to Test Position" suboption of the "Diagnostics" option of the main menu.

This distance can range from 0 to 90 in., but 30 in. is recommended. This gives a reasonably high count rate that gives a good precision in only a few seconds of counting, but there is no possibility of electronically saturating the detector tubes.

Acceleration. It is not necessary to move the cable with the highest possible acceleration for this check. An acceleration of $1000000 \mathrm{steps} / \mathrm{s}^{2}$ is recommended.

Velocity. It is not necessary to move the cable with the highest possible speed. The recommended velocity is a leisurely 50000 steps/s. 
General

(cont)
Item Specific Parameters. For each type of drum there is a separate set of parameters specific to that drum type. The proper parameters must be used for a type of drum and the first two characters in the drum's ID are used to make the selection.

Before discussing the parameters, a review of the assay and irradiation process may be helpful. An assay uses a set of "shuffles"; each shuffle consists of moving the source from the store position, irradiating the drum, returning the source to the store position, and counting delayed neutrons. The total number of delayed neutrons counted depends on the number of shuffles in an assay.

Each assay is preceded by a background count with the drum in place and the source stored.

For a fixed time in which to complete the assay, the amount of time used for the background count, the various times within a shuffle, and the number of shuffles in an assay must be selected. The recommended procedure is to select values that minimize the relative uncertainty in the assay result. The process for doing this is described in Appendix B.

Irradiation Strategy. The simplest way to irradiate a drum is to place the source across from the center of the drum for a few seconds. However, the ends of the drum will receive less irradiation than the center and if the density of uranium is not uniform throughout the drum a systematic error will have been introduced to the assay result. For example, if the density at one end is low and the irradiation of the end is also low, the assay result will be higher than it should. The sensitivity to inhomogeneity is poor with this simple irradiation scheme. 


\section{General}

(cont)
A second irradiation strategy is to bring the source quickly to the top of the drum and scan the source along the length of the drum during the irradiation time. Short pauses at the extremes of the scan can help make the irradiation uniform. This is the scanning strategy implemented for these shufflers.

The goal is to have generated a uniform distribution of delayed-neutron precursors when the source leaves for its storage position. The density of precursors will then track the density of uranium throughout the drum and the delayedneutron count rate will accurately follow the mass of uranium present.

This scanning strategy has several parameters. Where are the extreme positions of the scan relative to the drum's ends? How long should pauses be at the extreme positions? Some experimentation with a drum can be done to determine the optimum strategy.

The parameters of the next section include those needed for scanning: "Source Transfer Distance" to get the source just short of the drum quickly; "Pause Time at Scan's Top (Start)" and "Pause Time at Scan's Bottom (Far Point)" to give the ends extra irradiations; and "Number of Scans per Shuffle (One-Way)," "Scan Acceleration," "Scan Velocity," and "Scan Distance" to determine just how the scanning is to be done.

If scanning is not to be done, the "Number of Scans per Shuffle (One-Way)" should be set to zero. The irradiation time will be only the "Pause Time at Scan's Top (Start)." This is not a recommended mode of operation. 
General

(cont)
Assay Parameters. Some of these parameters are different for the X-326 and X-744G shufflers because different people made different choices a year or so apart. A comparison of assay values with the two sets of parameters has shown no noticeable effect; the same assay value resulted from the two sets of assay parameters. Either set of parameters could be used on both shufflers.

Transfer Distance (inches). This is the distance in inches the ${ }^{252} \mathrm{Cf}$ source will move rapidly toward the assay chamber to start an irradiation of a drum. The range of values is 0 to 99 in.; the actual distance depends on the geometry of the drum and the scanning procedure, but is likely to be close to 60 in. For a 32-in. high 55-gal. drum, the recommended distances are 56 in. for the X-326 shuffler and $60 \mathrm{in}$. for the $\mathrm{X}-744 \mathrm{G}$ shuffler.

Transfer Acceleration. This is the acceleration in motor steps $/ \mathrm{s}^{2}$ to be used in transferring the source toward the assay chamber to start an irradiation of a drum. This should have the largest value possible without causing the motor to stall; this is determined by trial and error. The possible values are from 0 to 9999 999; experience during development indicated a maximum acceleration for reliable motions to be 800000 steps $/ \mathrm{s}^{2}$.

Transfer Velocity. This is the velocity in motor steps/s to be used in transfering the source toward the assay chamber to start an irradiation of a drum. This should have the largest value possible without causing the motor to stall; this is determined by trial and error. The possible values are from 0 to 9999 999; experience during development indicated a maximum velocity for reliable motions to be 100000 steps/s. 
General

(cont)
Number of Shuffles Per Assay. This is the actual number of shuffles that will be used for an assay. This should be the same as the nominal number of shuffles per assay unless there is a very good reason to the contrary. The assay will be most accurate when the two numbers are the same.

The number can range from 1 to 90; the best value matches the nominal number of shuffles per assay. The number used during development of the X-326 shuffler was 28 while the number used for the X-744G shuffler was 34 . A comparison of the results from the two sets of assay parameters showed that the same assays results were obtained. The precision of an assay is improved by increasing the number of shuffles; the precision might be further improved by also extending the background count time.

Nominal Number of Shuffles Per Assay. This is the normal number of shuffles used in an assay and can range from 1 to 90. If the actual number of shuffles to be used is changed from this nominal number (perhaps to shorten the assay time), the delayed-neutron count rate will be adjusted automatically in the data analysis to simulate the result from using this nominal number. New calibration curves are thus not needed.

The accuracy of this adjustment is difficult to estimate and it is highly recommended that the number of shuffles actually used be equal to this nominal number.

Nominal Delayed-Neutron Count Time. The desired count time is entered here. The actual time is likely to deviate from this nominal time by a millisecond or so; differences this small are not important. The range of values is from 0 to $999.999 \mathrm{~s} ;$ during development a time of about $9 \mathrm{~s}$ was used and is recommended. 


\section{General}

(cont)
Nominal Irradiation Time. The expected time of irradiation of the drum during one shuffle is entered here. This entry does not determine the irradiation time used by the code; it is used only in calculating a correction factor for slight irregularities in timing and when the number of shuffles per assay is different from the nominal value.

The irradiation time is a combination of these scan parameters:

$$
\begin{aligned}
& \text { N = Number of Scans per Shuffle, } \\
& \text { D = Scan Distance, } \\
& \text { A = Scan Acceleration, } \\
& \text { V = Scan Velocity, } \\
& \text { PT = Pause Time at Scan's Top (Start), and } \\
& \text { PB = Pause Time at Scan's Bottom (Far Point). }
\end{aligned}
$$

It can be assumed that the scan has a constant velocity throughout because the acceleration is large and the velocity rather slow. The scan time is thus $S T=D / V$. The approximate irradiation time is then

$t_{l}=N(P T+S T+P B+S T)+P T$.

There is a final pause at the top before the irradiation is finished.

There is some additional time for transferring information to and from the Compumotor Indexer and within the computer's operating system, so the actual time is slightly greater than expected from the above list of parameters. 


\section{General \\ (cont)}

The best way to determine the Nominal Irradiation Time is to select a reasonable set of scan parameters and make a trial "assay" with the Full Printout turned on. The actual irradiation times will be printed. Use the average value of all the times as the Nominal Irradiation Time. (To quicken this process, nonessential parameters can be modified; select a short background count time and choose 5 or so shuffles per assay instead of 25.)

The range of possible values is 0 to $999.999 \mathrm{~s}$; during development, the scan parameters were selected so that the irradiation time was about $11 \mathrm{~s}$ and is recommended.

Nominal Forward Transfer Time. Based on values seen on full printouts, an expected transfer time for the source into the assay region is entered here. This is used along with the other nominal values to adjust the delayed-neutron count rate for irregularities in the source motions. The range of values is 0 to $999.999 \mathrm{~s}$. The actual value must be found from experience as displayed on long printouts and depends on the forward transfer distance, the acceleration, and the velocity. During development, with a transfer distance of 60 in., an average time of $1.988 \mathrm{~s}$ was found.

The forward transfer time includes data analysis and screen update times as well as the time needed to move the source into the irradiate position. It is thus longer than the nominal reverse transfer time. The forward transfer time for the first shuffle is always shorter than for the rest of the shuffles of an assay because there is no data analysis or screen update before moving the source for the first time.

The temptation to minimize transfer times by using the highest possible accelerations and velocities should be resisted. The assay result will not be improved to an important extent 
General

(cont) and the ${ }^{252} \mathrm{Cf}$ source transfer mechanism will undergo unnecessary wear. A forwand transfer time of 1.5 to $2.0 \mathrm{~s}$ is a satisfactory compromise time.

Nominal Reverse Transfer Time. Based on values seen on full printouts, an expected transfer time for the source from the irradiation position to the store position is entered here. The range of values is 0 to $999.999 \mathrm{~s}$. The actual value must be found from experience as displayed on long printouts and depends on the forward transfer distance, the acceleration, and velocity. During development, with a transfer distance of 60 in., the time of $0.780 \mathrm{~s}$ was found. This is actual time used in moving the source because there is no overhead time from calculations or screen updates as there is for the forward transfer time.

Scan Distance in Inches. This distance is related to the length of the drum, but is likely to be somewhat longer than the drum. The high-speed forward transfer distance may deliberately bring the source short of the first end of the drum. The scan distance should then be the length of the drum plus twice the short distance. The overscans on the drum's ends can give a more balanced irradiation to the ends relative to the drum's center.

A distance of zero is allowed, in which case no scanning is done; this is not recommended. The range of values is $\mathbf{0}$ to 9999.99 in. During development the scan distance was 33 in. for the X-326 shuffler and 29 in. for the X-744G shuffler.

Scan Acceleration (steps $/ \mathrm{s}^{2}$ ). The range of values is $\mathbf{0}$ to $9999999 \mathrm{steps} / \mathrm{s}^{2}$; the practical limit found during development was $500000 \mathrm{steps} / \mathrm{s}^{2}$ and is recommended. 
General

(cont)
Scan Velocity (steps/s). This need not be a large velocity. If the motor has 25000 steps/rev and the scan velocity is 25000 steps/s, the scan distance will be traversed at the speed of 8 in./s. If the scan distance is 12 in., the distance will be traversed in $1.5 \mathrm{~s}$. The range of values is 0 to 9999999 steps/s. The scan velocity depends on the scan distance, the motor's steps/rev, and the pause times at the top and bottom of the drums. For the X-326 shuffler, 150000 steps/s was used, while for the X-744G shuffler 14375 steps/s was used.

Number of Scans Per Shuffle (One-Way). This is the number of slow one-way scans that will be made through the drum's length during a single shuffle. Each one-way scan starts at one end of a drum and finishes at the other end. The purpose is to irradiate the drum as uniformly as possible with essentially a point source of neutrons. The nuniber of scans may be zero, implying that no scans will be done. The number of one-way scans should be an even number $(2,4,6$, ...) for best operation. The range of values is 0 to 998 .

During development the number of one-way scans was six for the X-326 shuffler and four for the X-744G shuffler because different pause times were used.

Pause Time at Scan's Top in Seconds. When the source is at the extreme of its scan nearest the store position, a pause can be made to irradiate the end of the drum. This can help balance the irradiation along the drum's length. A pause time of zero is allowed. The range of values is 0 to $999.9 \mathrm{~s}$.

During development, a time of $0.7 \mathrm{~s}$ was used with the X-326 shuffler and $1 \mathrm{~s}$ with the $\mathrm{X}-744 \mathrm{G}$ shuffler.

Pause Time at Scan's Bottom in Seconds. When the source is at the extreme of its scan farthest from the store position, a pause can be made to irradiate the end of the drum closest to 
General

(cont) the loors. This is an option that can help balance the irradiation along the drum's length. A pause time of zero is allowed. The range of values is 0 to $999.9 \mathrm{~s}$. During development, a time of $0.7 \mathrm{~s}$ was used with the $\mathrm{X}-326$ shuffler and $1 \mathrm{~s}$ with the $X-744 G$ shuffler.

Assay Backgic nd Count Time in Seconds. The time to be used for the passive background count at the start of an assay is specified here. The range of values is 0 to $999.9 \mathrm{~s}$; during development, a time of $270 \mathrm{~s}$ was usea.

Rotate During Assay. A drum should be rotated during an assay, so the response here should be "Y" for "yes."

Assay Diagnostics. After the assay data from a drum have been collected, some checks are automatically made to find obvious malfunctions. These parameters are needed for those checks.

Expected Delayed-Neutron Ratios. The counts from one bank of delayed-neutron detectors are compared to the counts from a symmetrically placed bank. Their ratio is expected to have the value specified by this parameter. The range of values is 0 to 99.9999 ; the ratio should be close to 1.00 .

If the actual ratio differs from the expected value by more than a specified percent, a malfunction is declared. An allowed deviation set too small will produce false malfunction indications; a value of $20 \%$ is recommended, but experience may lead to a better value.

Use Flux Monitors. This indicates if flux monitors are to be used in the data analysis. The usual purpose of flux monitors in shufflers is to adjust for varying amounts of 
General

(cont) moderating and absorbing materials in the assay chamber. It should be best to operate with the flux monitors and this parameter should be set to "Y" for "yes."

\section{Allowed Percent Deviation of the Cycle Correction} Factor.- As a check on the overall consistency of the shuffles in an assay, a cycle correction factor is computed that compares the effect of the actual timings to the effect of the nominal timings. If all the nominal values given above actually occur in an assay, the cycle correction factor is one. In practice, the timings may differ by a few milliseconds and the cycle correction factor is some number close to one (usually well within $0.5 \%$, depending on how well the nominal parameters match the actual values).

If the calculated cycle correction factor differs from unity by more than the percent given here, a malfunction is declared. This could indicate a problem with the timer or the source transfer system, or a number of shuffles per assay that differs greatly from the nominal number.

The range of values is 0 to $999.999 \%$; a value of $1 \%$ is recommended. If the cycle correction factor is less than 0.99 or greater than 1.01, first examine how well actual times match the nominal times; if there are differences, adjust the nominal times and see if the problem disappears. It should be possible to select parameters so that the cycle correction factor is routinely much less than $1 \%$ from unity.

Flux Monitor Dead Time in Microseconds. The dead time of the flux monitor detection electronics is given here and used to adjust the flux monitor count rate. The range of values is 0 to $9.99999 \mu \mathrm{s}$; it is recommended that the value be $0.9 \mu \mathrm{s}$. 
General

(cont)
Count rates in the delayed-neutron detector banks are too low to need a dead time correction.

Bias Correction. Bias Correction Parameters. There should normally be no need for a bias correction. But provision is made to correct the interpolated mass $m$ to a biascorrected mass $m$ (bias) in this manner: $m$ (bias) $=a_{2} m^{2}+$ $a_{1} m+a_{0}$. The range of values is -9999.99999 to 99999.99999.

Normally $a_{0}$ and $a_{2}$ will be zero and $a_{1}$ will be one, implying no bias correction.

Bias Correction Parameters' Variances and Covariances. There are three variances and three covariances to be entered. If there is no bias correction, all variances and covariances are zero.

Calibration Parameters. The user is first shown the number of enrichments that exist and is asked to select one enrichment for editing.

Number of Enrichments. Each drum type has a family of calibration curves; each curve corresponds to a different enrichment value. The maximum number of enrichments for a drum type is 10 . The actual number of enrichments for each type is entered through this suboption.

Which Enrichment Table. For each item type there may be more than one enrichment. Each enrichment has its own table of parameters. These parameters are then edited for each enrichment.

Enrichment Value. The value of the enrichment is given here. 
General

(cont)
Important Note: It is necessary that the data on the various calibration curves for a drum type be entered with ascending enrichments. For example, assume a drum type has four calibration curves at $5 \%, 15 \%, 27 \%$, and $58 \%$. The parameter "Number of Enrichments for Each Drum Type" is four. The Number 1 set of "Calibration Parameters" must correspond to the $5 \%$ enrichment, the Number 2 set to $15 \%$, and so on. If this procedure is not followed, the interpolation routine will give incorrect results.

Calibration Parameters. For each enrichment of each drum type, there are four calibration parameters ( $a_{0}$ through $a_{3}$ ) to be entered for this functional form: [corrected count rate] = $a_{3} m^{3}+a_{2} m^{2}+a_{1} m+a_{0}$, where the corrected count rate is in counts/s and $m$ is the drum's mass of $235 U$ in grams. Zeros for any of the parameters are allowed. The ranges of the parameters are -9999.99999 to 99999.99999 .

Calibration Parameters' Variances and Covariances. For each enrichment and each drum type, there are four variances and six covariances to be entered. If a parameter is set to zero because the corresponding dependence on mass is to be ignored in the calibration curve, the variance and the covariances involving that parameter should also be zero.

Normalization. Normalization Factor. This factor should be one unless there is a very good reason for another value. For example, if it is known that one delayed-neutron detector has gone dead and it is necessary to continue assays before repairs can be made, the normalization factor should be set to about $24 / 23$ so the calibration curves based on a full set of detectors still have validity. (In this example, the ratio of counts between the two banks will be affected and the expected ratio should be changed also.) 
General

(cont)

EXIT
Any need for a normalization factor other than one means that the normal precision and accuracy expected from the shuffler are probably no longer as good as they were before the problem arose.

The range of values is 0 to 999.999 , but without good cause the value should be set to 1.000 .

Uncertainty in the Normalization Factor. The uncertainty in the assay results will include whatever uncertainty is included here. A zero value is allowed. The range of values is 0 to 999.999; the routine value should be 0 and this is recommended.

This is identical to the EXIT option for an operator. 
GENERAL

USERS' PASSWORDS

EXIT
A manager can do all that a supervisor can do plus control the list of user identifiers and passwords. Therefore the Measurements, Safety Check, Archives, and Diagnostics options will not be described again.

Edit User Access Control. Only a manager can control the identifiers, passwords, and levels of access for the users. There may be more than one manager. The code supplied allows the maximum number of users to be 15 .

A table is presented to a manager, each line has a user name, the corresponding password, and the level of access. A level is indicated by a number: 0 for an operator, 1 for a supervisor, and 2 for a manager.

The information in the table can be edited by typing over the current information. The TAB and RETURN keys cause the cursor to move through the different fields of the table.

This is identical to the EXIT for an operator or a supervisor. 
GENERAL
GROSS COUNT RATES

This data analysis is performed after the data gathering process for an assay.

After the measurements for an assay are completed, the sums of the delayed-neutron counts in the eight scaler channels are $A D 1$ through $A D 8$ ( $A$ for assay, $D$ for delayed neutron, and a scaler channel number); each is a sum of the counts from each individual shuffle. The total delayed-neutron count is their sum: $A D=A D 1+A D 2+A D 3+A D 4+A D 5+A D 6$ $+A D 7+A D 8$. The total count time $T D$ is the sum of the individual shuffle's count times. The raw delayed-neutron count rate is $d_{\text {raw }}=A D / T D$.

The flux monitors are treated individually and then in combination. One is cadmium covered and the other is not. Their count rates are computed individually.

BACKGROUND SUBTRACTIONS The counts and the count time from the background portion of an assay have been retained by the code and are now subtracted from the gross count rates.

The background rate in the delayed-neutron detectors is $b_{d}=(B D 1+B D 2+B D 3+B D 4+B D 5+B D 6+B D 7$ $+B D 8) / T E$, where $B D x$ is a background count in delayedneutron channel $x$ and TB is the background count time. The background-corrected delayed-neutron count rate is thus $d_{1}=d_{\text {raw }}-b_{d}$.

The background rates in the flux-monitor detectors (cadmium covered and bare) are treated similarly but individually: $b_{f c}=B F 1 / T B$ and $b_{f b}=B F 2 / T B$. The background-corrected flux monitor-count rates are $f_{2 c}=f_{1 c}-b_{f c}$ and $f_{2 b}=f_{1 b}-b_{f b}$. 
${ }^{252}$ CI DECAY CORRECTION
The ${ }^{252} \mathrm{Cf}$ source decays exponentially with the decay constant $\lambda$ specified in the General parameter file. The calibration data were taken using a particular reference date for the source when the source had a certain size (the reference date might not be the date on which the calibration data were taken, but it could be). The current size of the source is smaller, so the count rates are adjusted to correspond to the size of the source on the reference date.

The decay-corrected count rates are $d_{2}=d_{1} e^{\lambda T}$ and $f_{2}=f_{1} e^{\lambda T}$, where $T$ is the number of days between the reference date and the measurement date.

FLUX MONITOR CORRECTION It is an option in the parameter file under Assay Diagnostics to use or not use a flux monitor correction. The normal purpose of a flux monitor is to adjust the count rates for variations in moderating and absorbing materials in the object being assayed.

If the matrix within a drum is known, and a calibration has been done using that matrix, it is more accurate not to use the flux monitor correction and simply to use the calibration curve.

If the matrix is not known, or a calibration has not been done with that matrix, the only option is to use the flux monitor correction and the calibration curve for unknown matrices.

The flux monitors count neutrons while the ${ }^{252} \mathrm{Cf}$ source is inside the assay chamber, so these count rates are much higher than rates from delayed neutrons. The $f_{2}$ is thus corrected for the loss of counts while the detector tubes recover from a previous neutron interaction: $f_{3 x}=f_{2 x} e^{\delta f_{1}}$, where $\delta$ is the detector's dead time and " $x$ " is either " $b$ " or " $c$." 
FLUX MONITOR CORRECTION The flux-monitor-corrected delayed-neutron count rate is (cont)

$d_{3}=d_{2} /\left(f_{B \text { are }} / f_{C d}\right)^{p\left(f_{B a r e} / f_{C d}\right)}$, where the function $p$ is $a_{2} r^{2}+a_{1} r+a_{0}$. The parameters in $p$ are not accessible through the Parameters menu option in the code. They should not be changed without an extensive study.

If the flux monitors are not used, then $d_{3}=d_{2}$.

CYCLE CORRECTION

Ideally the time durations of the various portions of an assay would not vary among assays. In practice they have slight variations; a large variation indicates a malfunction that should be called to the user's attention.

The cycle correction factor is calculated from the times measured for each activity of an assay and is compared to a reference value calculated with nominal values given in the parameter file. If the cycle correction factor differs from unity by more than a value set in the parameter file, a malfunction is suspected and an error message is given to the operator and associated with the assay result.

During the calibration measurements, a certain number of shuffles per assay was used for each measurement. It is best to continue using that number throughout all subsequent drum assays. But if for some reason a different number of shuffles is desired, the cycle correction factor will attempt to make up the difference. A change from 20 to 18 shuffles, for instance, will not give a correction factor much different from one and the accuracy of the results should not suffer much. However, changing from 20 to 5 shuffles will make the accuracy suspect because the cycle correction factor will be relatively large.

The cycle correction factor is $\left(D_{\text {nominal }} / D\right)$. $D_{\text {nominal }}$ is calculated using the nominal times and the number of shuffles 


\section{CYCLE CORRECTION} (cont) per assay; $D$ is a similar calculation but uses the actual num ber of shuffles and the times measured during each shuffle. If $D_{\text {nominal }}$ were multiplied by the fission rate induced in the material and by the delayed-neutron detection efficiency, it would be the calculated delayed-neutron count rate from an assay.

$$
\begin{aligned}
D_{\text {nominal }}= & \sum_{j=1}^{6} \frac{\beta_{j}}{\lambda_{j}}\left(1-e^{-\lambda_{j} t_{i}}\right)\left(e^{-\lambda_{j} t_{r}}\right)\left(1-e^{-\lambda_{j} t_{c}}\right) \\
& {\left[\frac{n}{1-e^{-\lambda_{j} \tau}}-\frac{e^{-\lambda_{j} \tau}\left(1-e^{-\lambda_{j} n \tau}\right)}{\left(1-e^{-\lambda_{j} \tau}\right)^{2}}\right] }
\end{aligned}
$$

The sum over $j$ is over the six groups of delayed-neutron precursors. The $\beta_{j}$ are the fractional yields of delayed neutrons from the six precursor groups and the $\lambda_{j}$ are the decay constants of the six groups. The nominal times for one shuffle are these: irradiation, $t_{i}$; reverse transfer to store position, $t_{r} ;$ count time, $t_{c}$; forward transfer to irradiate, $t_{f}$.

The nominal time for one shuffle is $\tau=t_{i}+t_{r}+t_{c}+t_{f}$. The nominal number of shuffles per assay is $n$.

A term contributing to $D$ is calculated after each shuffle of an assay. The actual times during shuffle $s$ are $t_{i s}, t_{r s}, t_{c s}, t_{f s}$; their sum is $\tau_{s}$. After shuffle number $s$, the new term for $D$ is this sum over the six precursor groups: 


\section{CYCLE CORRECTION}

(cont)

$$
\begin{aligned}
& D(s)=\sum_{j=i}^{6} P_{j}(s)\left(1-e^{-\lambda_{j} t_{c s}}\right) \\
& \text { where } \\
& P_{j}(s)=\frac{\beta_{j}}{\lambda_{j}}\left(1-e^{\lambda_{j} t_{i s}}\right)\left(e^{-\lambda_{j} t_{r s}}\right)+P_{j}^{\prime}(s-1) e^{-\lambda_{j}\left(t_{f s}+t_{i s}+t_{r s}\right)} \\
& P_{j}^{\prime}(s)=P_{j}^{\prime}(s-1) e^{-\lambda_{j} \tau_{s}}+\frac{\beta_{j}}{\lambda_{j}} e^{-\lambda_{j}\left(t_{r s}+t_{c s}\right)}\left(1-e^{\lambda_{j} t_{i s}}\right) \\
& P_{j}^{\prime}(0)=0
\end{aligned}
$$

$D$ is the sum of the $D(s)$ over all $s$.

The cycle-corrected count rate is $d_{4}=d_{3}\left(D_{\text {nominal }} D\right)$.

NORMALIZATION

INTERPOLATION
The normalization correction should routinely be one, implying that no normalization is applied.

The normalization-corrected delayed-neutron count rate is $d_{5}=N F d_{5}$.

From the corrected count rate $d_{5}$, the mass of ${ }^{235} \mathrm{U}$ in the drum is interpolated from calibration curves. It is required 
INTERPOLATION

(cont) that the calibration data used to generate the curves encompass the enrichment and mass of the drum. This means that extrapolations will not be done.

The general expression for the interpolation relates the corrected count rate $d_{5}$ to the mass of $235 \mathrm{U}$ in a drum. From the corrections described just above, this expression is

$$
\begin{aligned}
d_{5}= & \left(d_{\text {raw }}-b_{d}\right) e^{\lambda T}\left(f_{B \text { are }} / f_{C d}\right)^{-p\left(f_{B a r e} / f_{C d}\right)} \\
& \left(D_{\text {nominal }} / D\right)(N F)=f(m,\{a\})
\end{aligned}
$$

where $f(m,\{a\})$ represents the calibration function with the mass $m$ and a set of parameters $\{a\}$. In this case, the calibration function is a third-order polynomial.

Calibration for a type of drum uses a set of drums containing different $235 U$ masses at each of a set of ${ }^{235} U$ enrichments. From the corrected count rates $d_{5}$ from each drum, a family of curves is generated for that type of drum; each curve is for a particular enrichment.

The interpolation of an assay result $m_{i}$ from a new $d_{5}$ begins by selecting the family of curves according to the drum type. All the curves have the same functional form, but each curve has its own set of parameters. From the count rate $d_{5}$ two masses $\left(m_{1}\right.$ and $\left.m_{2}\right)$ are interpolated from the calibration curves whose enrichments $\left(e_{1}\right.$ and $\left.e_{2}\right)$ bracket the enrichment of the drum. Another linear interpolation is then made between $m_{1}$ and $m_{2}$ on the enrichment to get a single mass corresponding to the count rate and the enrichment of the drum. 


\section{BIAS CORRECTION}

ASSAY UNCERTAINTIES

\section{Random Uncertainty}

This correction should not be needed routinely. Despite all the above corrections the assay results might still be in error by some predictable amount. The cause may or may not be understood, but a bias correction factor $B C F$ in the form of a second-order polynomial is provided.

The final 235U mass in a drum is then $m_{\text {final }}=g(m,\{a\})$, where $\{a\}$ is a set of parameters for the bias correction function.

Three uncertainties are calculated for each assay: random $\left(\sigma_{r}\right)$, systematic $\left(\sigma_{s}\right)$, and total $\left(\sigma_{t}\right)$. They are related through this sum: $\left(\sigma_{t}\right)^{2}=\left(\sigma_{r}\right)^{2}+\left(\sigma_{s}\right)^{2}$.

Random uncertainties arise forming fluctuations that are inherent and unavoidable because of the nature of the items being measured. The random uncertainty is often called the precision of the instrument.

A shuffler has several sources of random uncertainties. Some arise from factors in the expression for $d_{5}$ given above: there are fluctuations in the count rates of delayed neutrons and their backgrounds and also in the measured count rates from flux monitors and their backgrounds (assuming a flux monitor correction is made). More difficult to assess are uncertainties in the correction for the decay of $252 \mathrm{Cf}$, in the measured times in the cycle correction, and mechanical variations.

The uncertainty in the correction factor for the decay of $252 \mathrm{Cf}$ is taken as a random uncertainty here for the following reasons. If the source of error were simply an imprecise value of the decay constant, the uncertainty would be systematic. That error is assumed to be negligible. The other source of error is in calculating the number of days since the 
Random Uncertainty (cont)
Systematic Uncertainty reference date. The algorithm uses an integer number of days for the interval and thus an assay in the evening has the same decay correction factor as an assay in the morning. The amount of error depends on the time of day. Fortunately, the magnitude of the error is quite unimportant compared to other uncertainties.

The cycle correction factor uses the measured times for various segments of an assay. These times no doubt have random variations that lead to random uncertainties in $d_{55}$. It is difficult to determine the size of the variations in the times, but repeated runs that display the times have shown them to rarely be more than a millisecond and thus these variations lead to unimportant variations in $d_{5}$. This uncertainty is ignored in this error analysis.

Mechanical variations arise from differences in the geometries used in assays. The ${ }^{252} \mathrm{Cf}$ source may not stop in exactly the same location every time even though the same number of motor steps was taken; the flexible cable to which it is attached may bend slightly differently within the guide tube from one time to another, or it may spiral through the gear teeth a short distance. In practice these variations have been found to be very small. If the calculated random uncertainty is the same as the measured precision of the instrument, these mechanical variations are having no significant effect; this has been the experience with recent shufflers.

Systematic uncertainties arise from the corrections to the data and from interpretations given to the data. The systematic uncertainty is sometimes called the bias; the word "bias" is also used with other meanings, so its meaning should be carefully sought in the context of its use. 
Systematic Uncertainty (cont)

Total Uncertainty
The most important source of systematic uncertainty is the calibration process. Any error in the calibration curves will affect all assays that use those curves.

If a normalization factor or a bias correction other than unity are applied, it too will introduce systematic uncertainties into all the assay results.

The total uncertainity is the square root of the sum of the squares of the random and systematic uncertainties. An expression for the total uncertainty is developed below and then expressions for the random and systematic uncertainties are taken from it.

Using the explicit expressions for the various functions, the relation between the count rate and the ${ }^{235} \mathrm{U}$ mass is

$$
\begin{aligned}
d_{5}= & \left(d_{\text {raw }}-b_{d}\right) e^{\lambda T}\left(f_{B \text { are }} / f_{C d}\right)^{-p\left(f_{\text {Bare }} / f_{C d}\right)}\left(D_{\text {nominal }} / D\right) \\
& (N F)=a_{3} m^{3}+a_{2} m^{2}+a_{1} m+a_{0} .
\end{aligned}
$$

The uncertainty in $m$ is desired and the variance of $m$ has this expression:

$$
\sigma_{m_{k}}^{2}=\left(\frac{\partial m_{k}}{\partial d_{5}}\right)^{2} \sigma_{d_{5}}{ }^{2}+\sum_{i} \sum_{j}\left(\frac{\partial m_{k}}{\partial a_{i}}\right)\left(\frac{\partial m_{k}}{\partial a_{j}}\right) \sigma_{a_{i} a_{j}}
$$

The $\sigma_{i j}$ are covariances if $i$ is not equal to $j$, and are variances if $i$ equals $j$; these are obtained from a curve-fitting procedire used to find the parameters $\alpha_{i}$. The partial derivatives can be expanded into simple expressions. The two 
Total Uncertainty (cont) derivatives of $m$ are evaluated first, followed by the more complex variance of $d 5$.

$$
\begin{aligned}
& \frac{\partial m_{k}}{\partial d_{5}}=\frac{1}{\frac{\partial d_{5}}{\partial m_{k}}}=\frac{1}{\frac{\partial f}{\partial m_{k}}} \\
& \frac{\partial m_{k}}{\partial a_{i}}=\left(\frac{\partial m_{k}}{\partial d_{5}}\right)\left(\frac{\partial d_{5}}{\partial a_{i}}\right) \\
& \sigma_{d_{5}}^{2}=\left(\frac{\partial d_{5}}{\partial d_{\text {raw }}}\right)^{2} \sigma_{\text {raw }}^{2}+\left(\frac{\partial d_{5}}{\partial b_{d}}\right)^{2} \sigma_{b_{d}}^{2}+\left(\frac{\partial d_{5}}{\partial N F}\right)^{2} \sigma_{N F}^{2}
\end{aligned}
$$

The variance of $d_{5}$ depends on variances from all the factors in tue expression for $d_{5}$.

$$
\begin{aligned}
\sigma_{d_{5}}^{2} & =\left(\frac{d_{5}}{d_{\text {raw }}-b_{d}}\right)^{2}\left(\frac{d_{\text {raw }}}{T D}+\frac{b_{d}}{T B}\right)+\left(\frac{d_{5}}{N F}\right)^{2} \sigma_{N F}^{2} \\
\sigma_{f_{3}}^{2} & =\left(\frac{\partial f_{3}}{\partial f_{\text {raw }}}\right)^{2} \sigma_{f_{\text {raw }}}^{2}+\left(\frac{\partial f_{3}}{\partial b_{f}}\right)^{2} \sigma_{b_{f}}^{2} \\
& +\left(\frac{f_{3}}{f_{\text {raw }}-b_{f}}\right)^{2}\left(\frac{f_{\text {raw }}}{T D}+\frac{b_{f}}{T B}\right)
\end{aligned}
$$

This general variance expression can be split into random and systematic terms. 


\section{Total Uncertainty}

(cont)

$$
\begin{aligned}
\sigma_{r}^{2}= & \frac{1}{\left(\frac{\partial f}{\partial m_{k}}\right)^{2}}\left[\left(\frac{d_{5}}{d_{\text {raw }}-b_{d}}\right)^{2}\left(\frac{d_{\text {raw }}}{T D}+\frac{b_{d}}{T B}\right)\right. \\
& \left.+\left(\frac{d_{5}}{f_{3_{\text {ref }}}}\right)^{2} \sigma_{f_{\text {ref }}}^{2}+\left(\frac{d_{5}}{f_{3}}\right)^{2}\left(\frac{f_{3}}{f_{\text {raw }}-b_{f}}\right)^{2}\left(\frac{f_{1}}{T D}+\frac{b_{f}}{T B}\right)\right]
\end{aligned}
$$

$$
\sigma_{s}^{2}=\frac{\left(\frac{d_{5}}{N F}\right)^{2} \sigma_{N F}^{2}}{\left(\frac{\partial f}{\partial m_{k}}\right)^{2}}+\sum_{i} \sum_{j}\left(\frac{\partial m_{k}}{\partial a_{i}}\right)\left(\frac{\partial m_{k}}{\partial a_{j}}\right) \sigma_{a_{i} a_{j}}
$$

The variance from the cycle correction factor is taken to be zero because of the good precision in the timing of shuffler events.

The uncertainty in the mass is thus

$$
\sigma_{m}=\sqrt{\sigma_{r}^{2}+\sigma_{s}^{2}}
$$

If a bias correction is necessary, it introduces additional systematic uncertainty. The bias function is $g(m,\{a\})$ and has parameters $b_{i}$ with covariances $\sigma_{a_{i} a_{j}}$. The uncertainty in the bias-corrected mass is 
Total Uncertainty

(cont)

$$
\sigma_{B}^{2}=\left(\frac{\partial f_{B}}{\partial m}\right)^{2} \sigma_{m}^{2}+\sum_{i} \sum_{j}\left(\frac{\partial f_{B}}{\partial b_{i}}\right)\left(\frac{\partial f_{B}}{\partial b_{j}}\right) \sigma_{b_{i} b_{j}}
$$

FULL PRINTOUT

When the Full Printout option under Diagnostics is selected, all the raw data and the intermediate values of the data analysis process are sent to the printer. The purpose of the example given next is to show the format of the Full Printout; the numbers shown are not necessarily typical nor should be expected from assays.

Measurement type: ASSAY drum ID: TEST 19-Nov-1991 13:35:05

\section{BACKGROUND RAW DATA}

Background count time $=269.402400$

Background flux monitor 1 Counts $=14$

Background flux monitor 2 Counts $=9$

Background delayed neutron 1 Counts $=887$

Background delayed neutron 2 Counts $=762$

Background delayed neutron 3 Counts $=721$

Background delayed neutron 4 Counts $=775$

Background delayed neutron 5 Counts $=861$

Background delayed neutron 6 Counts $=828$

Background delayed neutron 7 Counts $=1958$

Background delayed neutron 8 Counts $=1031$

\section{RAW DATA FOR EACH OF 34 SHUFFLES}

Forward Time $(s)=0.776600$ Irradiation Time $(s)=11.272700$

Reverse Time $(s)=0.783400 \quad$ Count Time $(s)=7.916400$

Flux Monitor 1 Counts $=2173598$

Flux Monitor 2 Counts $=1282356$ 


\section{FULL PRINTOUT}

(cont)

Delayed Neutron 1 Counts $=61$

Delayed Neutron 2 Counts $=55$

Delayed Neutron 3 Counts $=54$

Delayed Neutron 4 Counts $=59$

Delayed Neutron 5 Counts $=63$

Delayed Neutron 6 Counts $=62$

Delayed Neutron 7 Counts $=74$

Delayed Neutron 8 Counts $=44$

Forward Time $(s)=1.997800 \quad$ Irradiation Time $(s)=11.254900$

Reverse Time $(s)=0.774700 \quad$ Count Time $(s)=7.478800$

Flux Monitor 1 Counts $=2170100$

Flux Monitor 2 Counts $=1281584$

Delayed Neutron 1 Counts $=61$

Delayed Neutron 2 Counts $=64$

Delayed Neutron 3 Counts $=64$

Delayed Neutron 4 Counts $=67$

Delayed Neutron 5 Counts $=49$

Delayed Neutron 6 Counts $=52$

Delayed Neutron 7 Counts $=76$

Delayed Neutron 8 Counts $=42$

Forward Time $(s)=1.879300 \quad$ Irradiation Time $(s)=11.271300$

Reverse Time $(s)=0.779100 \quad$ Count Time $(s)=7.576500$

Flux Monitor 1 Counts $=2169659$

Flux Monitor 2 Counts $=1281992$

Delayed Neutron 1 Counts $=55$

Delayed Neutron 2 Counts $=71$

Delayed Neutron 3 Counts $=62$

Delayed Neutron 4 Counts $=79$

Delayed Neutron 5 Counts $=71$

Delayed Neutron 6 Counts $=48$

Delayed Neutron 7 Counts $=73$

Delayed Neutron 8 Counts $=42$ 


\section{FULL PRINTOUT}

(cont)

... [shuffles 4 through 33 are not shown here; the following is shuffle 34]

Forward Time $(s)=2.13800000 \quad$ Irradiation Time $(s)=11.267600$

Reverse Time $(s)=0.779100 \quad$ Count Time $(s)=7.321500$

Flux Monitor 1 Counts $=2168657$

Flux Monitor 2 Counts $=1284331$

Delayed Neutron 1 Counts $=74$

Delayed Neutron 2 Counts $=71$

Delayed Nentrin 3 Counts $=82$

Delayed Neutron 4 Counts $=46$

Delayed Neutron 5 Counts $=54$

Delayed Neutron 6 Counts $=60$

Delayed Neutron 7 Counts $=66$

Delayed Neutron 8 Counts $=39$

SUMMED DATA FOR 34 SHUFFLES

Forward Time $(s)=54.067800 \quad$ Irradiation Time $(s)=382.962400$

Reverse Time $(s)=26.442600$ Count Time $(s)=257.207100$

Flux Monitor 1 Counts $=73780831$

Flux Monitor 2 Counts $=43602093$

Total Flux Monitor Counts $=117382924$

Delayed Neutron 1 Counts $=2316$

Delayed Neutron 2 Counts $=2299$

Delayed Neutron 3 Counts $=2228$

Delayed Neutron 4 Counts $=2171$

Delayed Neutron 5 Counts $=2283$

Delayed Neutron 6 Counts $=2416$

Delayed Neutron 7 Counts $=2646$

Delayed Neutron 8 Counts $=1491$

Total Delayed Neutron Counts $=17850$

\section{RAW COUNT RATES AND UNCERTAINTIES}

Flux Monitor Ratio (1:2) $\quad=1.692140$

Delayed Neutron Ratio $(1: 1)=1.000000$ 


\section{FULL PRINTOUT}

(cont)

Delayed Neutron Ratio $(1: 1)=1.000000$

Delayed Neutron Ratio $(1: 1)=1.000000$

Delayed Neutron Ratio (1:1) $=1.000000$

Delayed Neutron Rate (cps) $\quad=69.399+/-0.519$

\section{RESPONSE CORRECTIONS}

BACKGROUND and Cf-252 DECAY

Flux Monitor Background Rate (cps) $=0.085$

Delayed Neutron Background Rate (cps) $=29.04$

Background Corrected Flux Monitor Rate (cps) $=306512.84$

Background Corrected Delayed Neutron Rate (cps) $=40.361$

Reference Date $=$ 26-SEP-90 Current Date $=19-$ Nov-1991 13:35:05

Number of Days Since Reference Date $=419.55$

Decay Constant $(1 / \mathrm{d})=0.0007130$

Decay Correction Factor $=1.348700$

Cf Decay Corrected Flux Monitor Rate (cps) $=413393.98$

Cf Decay Corrected Delayed Neutron Rate (cps) $=54.435$

\section{DEAD TIME}

Delta (microseconds) $=0.900$

Dead Time Correction Factor $=1.3176654$

Dead Time Corrected Flux Monitor Rate = 544714.9538244

\section{FLUX MONITOR}

Flux Monitor Correction Factor $=5.341$

Flux Monitor Corrected Delayed Neutron Rate (cps) $=54.435$

\section{CYCLE CORRECTION}

Nominal Number of Shuffles per Assay $=34$

Nom. Fwrd. Time $(s)=1.988000 \quad$ Nom. Irrad. Time $(s)=11.262000$

Nom. Rev. Time (s) $=0.780000 \quad$ Nom. Count Time $(s)=7.007000$ 


\section{FULL PRINTOUT}

(cont)

$\operatorname{Dsum}($ nominal $)=0.55850$

Dsum $=0.58301$

Correction Factor $=0.95796$

Cycle Corrected Delayed Neutron Count Rate (cps) $=52.1463$

\section{NORMALIZATION}

Normalization factor $=1.00000+/-0.00000$

Normalization corrected delayed neutron count rate $=52.14626$

\section{INTERPOLATION}

$c=a 3 m^{\wedge} 3+a 2 m^{\wedge} 2+a 1 m+a 0$

e1 $=94.50000$ a $0=0.00000$ a1 $=13.33000$ a2 $=0.00000$ a3 $=0.00000$

$\operatorname{CV}[0,0]=0.000 \mathrm{e}+00 \quad \mathrm{CV}[0,1]=0.000 \mathrm{e}+00 \quad \mathrm{CV}[0,2]=0.000 \mathrm{e}+00 \quad \mathrm{CV}[0,3]=0.000 \mathrm{e}+00$

$\operatorname{CV}[1,0]=0.000 \mathrm{e}+00 \quad \mathrm{CV}[1,1]=1.689 \mathrm{e}+01 \quad \mathrm{CV}[1,2]=0.000 \mathrm{e}+00 \quad \mathrm{CV}[1,3]=1.000 \mathrm{e}+00$

$\operatorname{CV}[2,0]=0.06 \mathrm{Cl}+00 \quad \mathrm{CV}[2,1]=0.000 \mathrm{e}+00 \quad \mathrm{CV}[2,2]=0.000 \mathrm{e}+00 \quad \mathrm{CV}[2,3]=0.000 \mathrm{e}+00$

$\operatorname{CV}[3,0]=0.000 \mathrm{e}+00 \quad \mathrm{CV}[3,1]=1.000 \mathrm{e}+00 \quad \mathrm{CV}[3,2]=0.000 \mathrm{e}+00 \quad \mathrm{CV}[3,3]=1.000 \mathrm{e}+0$

$\mathrm{e} 2=94.50000 \mathrm{a} 0=0.00000 \mathrm{a} 1=13.33000 \mathrm{a} 2=0.00000 \mathrm{a} 3=0.00000$
$\mathrm{CV}[0,0]=0.000 \mathrm{e}+00$
$\mathrm{CV}[0,1]=0.000 \mathrm{e}+00$
$\mathrm{CV}[0,2]=0.000 \mathrm{e}+00$
$\mathrm{CV}[0,3]=0.000 \mathrm{e}+00$
$\mathrm{CV}[1,0]=0.000 \mathrm{e}+00$
$\mathrm{CV}[1,1]=1.689 \mathrm{e}+01$
$\mathrm{CV}[1,2]=0.000 \mathrm{e}+00$
$\mathrm{CV}[1,3]=1.000 \mathrm{e}+00$
$\mathrm{CV}[2,0]=0.000 \mathrm{e}+00$
$\operatorname{CV}[2,1]=0.000 \mathrm{e}+00$
$\mathrm{CV}[2,2]=0.000 \mathrm{e}+00$
$\mathrm{CV}[2,3]=0.000 \mathrm{e}+00$
$\mathrm{CV}[3,0]=0.000 \mathrm{e}+00$
$\operatorname{CV}[3,1]=1.000 \mathrm{e}+00$
$\mathrm{CV}[3,2]=0.000 \mathrm{e}+00$
$\operatorname{CV}[3,3]=1.000 e+0$
$\mathrm{m} 1=3.91195$

Uncertainties: random $=0.0596$ systematic $=1.2061$ total $=1.2076$ $\mathrm{m} 2=541.21797$

Uncertainties: random $=0.0596$ systematic $=1.2061$ total $=1.2076$ drum e $=94.5000$

Interpolated Mass $(\mathrm{g})=3.9119$

Interpolated Sigma $=1.2076$

\section{BIAS CORRECTION TO ASSAY}

Bias Correction Mass $=\mathrm{a} 3 \mathrm{~m}^{\wedge} 3+\mathrm{a} 2 \mathrm{~m}^{\wedge} 2+\mathrm{a} 1 \mathrm{~m}+\mathrm{a} 0$ $\mathrm{a} 2=0.00000$ a1 $=1.00000$ a0 $=0.00000$ 


\section{FULL PRINTOUT \\ (cont) \\ $\mathrm{CV}[0,0]=1.0000 \mathrm{CV}[0,1]=0.0000 \quad \mathrm{CF}[0,2]=0.0000$ \\ $\mathrm{CV}[1,0]=0.0000 \quad \mathrm{CV}[1,1]=1.0000 \mathrm{CF}[1,2]=0.0000$ \\ $\mathrm{CV}[2,0]=0.0000 \quad \mathrm{CV}[2,1]=0.0000 \quad \mathrm{CF}[2,2]=1.0000$}

Bias Corrected Mass $(\mathrm{g})=3.9119+/-1.2076$

The first line of the long printout gives the type of measurement, the ID of the drum, and the date and time.

The type of measurement in this case is ASSAY, but it could be BIAS AND PRECISION, PRECISION, or CALIBRATION.

The assay result and error status code are given on line 2 .

These first two lines are normally printed even if a full printout is not requested.

The BACKGROUND RAW DATA shows the count time, the counts from each flux monitor, and the counts from each of the delayed-neutron detector banks.

Each shuffle of the assay has its own set of times and counts. Each set is printed under the heading RAW DATA FOR EACH OF 34 SHUFFLES. (The number "34" will be the number specified in the Assay Parameters form of the parameter file.) The measured times to move the source forward, to irradiate, to move in reverse, and to count are printed. The counts in the flux monitors are done during the irradiation time; the counts in the delayed-neutron detectors are done during the count time. 
FULL PRINTOUT (cont)
The forward time of the first shuffle will always be shorter than the forward times of all the following shuffles because there is less "overhead" (storing the source, doing calculations, writing to disk) for the first shuffle. There are no adverse effects from this minor timing irregularity.

Also note that the delayed-neutron count rate increases as the shuffles progress. More delayed neutrons are counted in each shuffle as there are more previous shuffles that have generated long-lived precursor nuclei. An equilibrium rate is approached as the assay progresses.

The times to perform the various actions and the counts gathered during each shuffle are added together and printed as SUMMED DATA FOR 34 SHUFFLES.

From the summed data, the RAW COUNT RATES AND UNCERTAINTIES are calculated. Ratios of counts are taken for the flux monitors and delayed-neutron detector banks. The ratio of the "bare" to "Cd-covered" flux monitor counts is given first. The banks used in the next four ratios depend on the entry made by a supervisor or manager in the Assay Diagnostics form of the parameter file. In this example, bank 1 was always compared to itself. It is more useful to compare banks 1 and 6 (the doors), banks 2 and 5 (sides near the front), and banks 3 and 4 (sides near the back). The fourth ratio should duplicate one of those just given; it is not reasonable to use banks 7 and 8 (top and bottom) as a ratio because the matrix material and the vertical position of the fissile material strongly affect this ratio, making it highly variable. The delayed-neutron rate is the sum of the counts from all the delayed-neutron channels divided by the summed count time. 
FULL PRINTOUT

(cont)
Various RESPONSE CORRECTIONS are now performed. The effect of each is given. This is especially useful in checking the data analysis step by step.

The BACKGROUND AND Cf-252 DECAY corrections are applied to both the flux monitor and delayed-neutron count rates. The measured background rates from the flux monitors and the delayed-neutron detectors are given and then subtracted from the raw count rates to give the background corrected count rates.

The correction for the decay of the ${ }^{252} \mathrm{Cf}$ source is done by adjusting the count rates to what would have been measured on a reference date specified in the General parameter file. The Reference Date is printed along with the Current Date.

The correction for the source decay proceeds by finding the number of days $T$ between the reference date and the current date and calculating $e^{\lambda T}$, where $\lambda$ is the decay constant (in units of $\left.d^{-1}\right)$. This exponential is the Decay Correction Factor shown on the printout. This factor is then a multiplier of the two count rates with the effects shown on the printout.

The high-count rates in the flux monitors during irradiation mean that some neutrons will be missed because the detector was "dead," that is, had not recovered from the previous neutron interaction. The DEAD TIME correction factor makes up for this loss. From the raw flux monitor count rate $f_{1}$, the correction factor is $e^{\delta f_{1}}$, where $\delta$ is the dead time parameter in the General parameter file. The long printout shows $\delta$ in microseconds, the correction factor, and the total flux monitor count rate after the correction factor has been applied. 
FULL PRINTOUT

(cont)
The FLUX MONITOR correction is a method of adjusting the delayed-neutron count rates for the effects of varying matrix (nonfissile) materials. A supervisor can set a parameter so that the flux monitor correction is not applied to the delayed-neutron data. That choice was made in this example so that even though the flux monitor correction factor is 5.341, the Flux Monitor Corrected Delayed-Neutron Count Rate is unchanged from the Cf Decay Corrected DelayedNeutron Rate.

The CYCLE CORRECTION is a technique to monitor the beha rior of the shuffler by comparing the results of the acr dal event times to the results of the expected event times. A formula using the nominal times in the parameter file calculates the expected count rate, aside from a constant that depends on the source strength and detection efficiency; the same formula is then used with the actual times to get a second value. The ratio of these two calculated values is the Correction Factor. If the nominal times match the actual times, the Correction Factor will be less than $1 \%$ away from one. The printout shows the nominal number of shuffles in the assay and the nominal times. Dsum(nominal) is the value calculated with the nominal values, while Dsum is the same after using uite measured values. The Correction Factor in this case is farther from 1 than necessary, meaning that better choices of the nominal times could be made. The Correction Factor is then applied to the delayed-neutron count rate and the result is printed.

NORMALIZATION is provided but should rarely (if ever) be needed to modify the count rate. The normalization factor here is one (with no uncertainty) and does not change the count rate (or contribute to the uncertainty). 
FULL PRINTOUT

(cont)
The results of INTERPOLATION on the calibration curves are shown. The form of the curve is printed along with enrichments and corresponding sets of parameters (with their covariances). In this example, there was really only one enrichment curve; both are for $94.5 \%$ enrichment and that is the same as the object being assayed. The two masses, $m 1$ and $m 2$, are the same because the two calibration curves are the same. When there are two different calibration curves, there will be two different interpolated masses ( $m 1$ and $m 2$ ) corresponding to the two enrichments ( $e 1$ and $e 2)$ that flank the enrichment for the drum $(e)$. The Interpolated Mass in this example is the same as $m 1$ and $m 2$ because $e=e 1=e 2$.

The BIAS CORRECTION TO ASS $\triangle Y$ will generally have no effect on the assay's mass because the parameters are set to simply multiply the previous mass by one. The capability of doing a bias correction is provided but it is not expected to be necessary. On the printout the form of the Bias Correction Factor is given along with its parameters and covariances, as set by a supervisor or manager in the Bias Correction parameter file. The Bias Corrected Mass is the final result for the mass of an assay. 
GENERAL
The two measurement options of Standard Check and Bias and Precision Checks involve statistical tests to determine if the checks were passed or failed. Details on these tests are presented in this section. The techniques are taken from these three references:

1. S. -T. Hsue and T. Marks, "Measurement Control Program for NDA Instruments," in Proceedings of the Conference on Safeguards Technology: the ProcessSafeguards Interface (U.S. Department of Energy, Chicago Operations Office, New Brunswick Laboratory, Argonne, IL, 1984), pp. 422-425.

2. A. S. Goldman and R. S. Marshall, "Fine Tuning of a Measurement Control Program at the Los Alamos National Laboratory," Proceedings of the Conference on Safeguards Technology: the Process-Safeguards Interface (U.S. Department of Energy, Chicago Operations Office, New Brunswick Laboratory, Argonne, IL, 1984), pp. 426-432.

3. M. G. Natrella, Experimental Statistics, National Bureau of Standards Handbook 91 (U.S. Department of Commerce, National Bureau of Standards, 1963).

When a test with a confidence level is used, it is useful to know that a higher confidence level makes the test easier to pass. The range of acceptable values is wider at $99 \%$ confidence than at $95 \%$ confidence; a $65 \%$ confidence range is much more narrow than a $95 \%$ confidence range, so the test is more stringent. Common practice uses $95 \%$ confidence, but experience with false failure rates may dictate ancther value. 


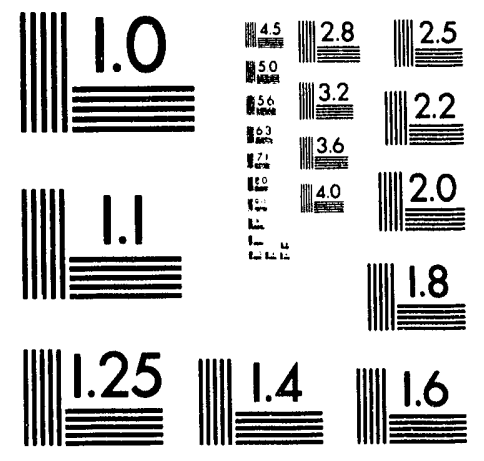



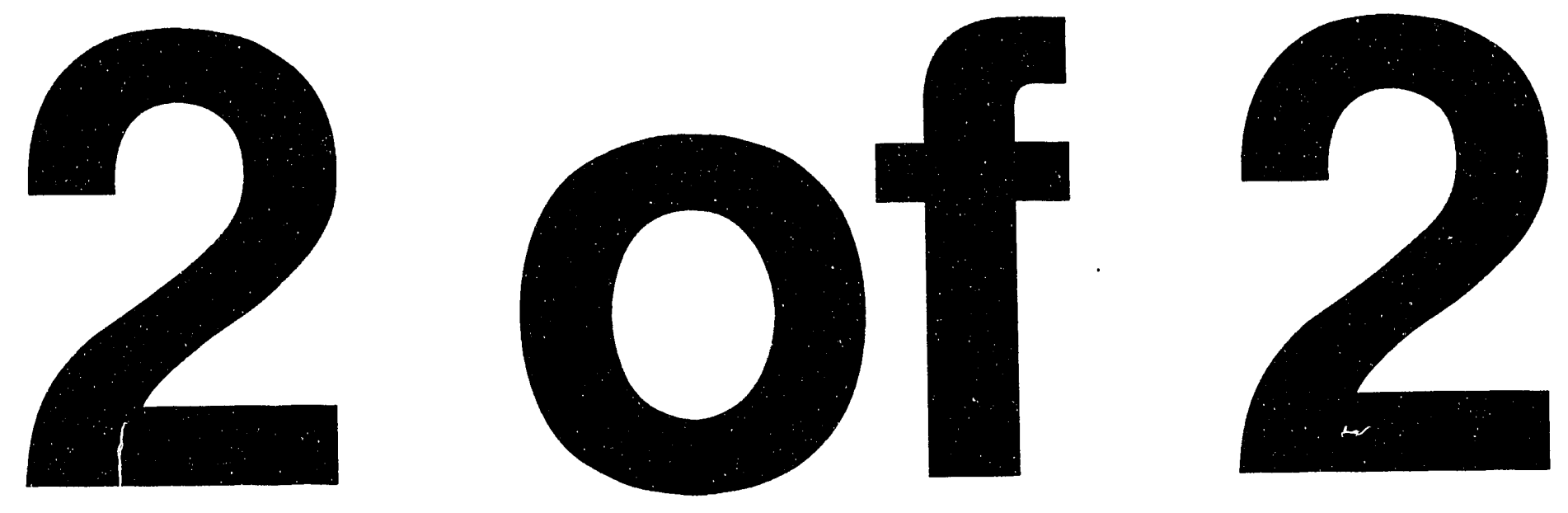
STANDARD CHECK
The assay result with a standard drum is expected to be $m_{\text {std }}$, with a percent confidence level of $c$ (typically 95\%). The actual measurement result is $m \pm \sigma$.

The test for agreement between $m_{\text {std }}$ and $m$ uses a value of Student's $t$ for the confidence level $c$ and an infinite number of degrees of freedom $d f$ (well approximated by 1000 in the code).

If the percent confidence level is $c$, the fractional confidence level is $c^{\prime}=c / 100$. Tables of Student's $t$ deal with the parameter $\alpha$, which in this case is related to $c$ by

$(1-\alpha)=c^{\prime}$

The corresponding $t$ has the parameter

$(1-\alpha / 2)=(1+c) / 2$.

For example, if $c=95 \%$ then $c^{\circ}=0.95, \alpha=0.05$, and ( 1 $\alpha / 2)=0.975$. If a table of Student's $t$ is consulted, there will be a column labeled t0.975 and a row for infinite degrees of freedom; the value of $t$ is 1.960 .

A table of selected $t$ values is not built into the code for the shuffler. A calculation is available that is more flexible; the user is not limited to one particular confidence level. The formula is from M. Abramowitz and I. A. Stegun, Handbook of Mathematical Functions, (Dover Publications, Inc., New York, 1965), p. 949, Eq. 26.7.5. 


\section{STANDARD CHECK}

(cont)
The calculation of Student's $t$ requires the width of the central portion of a normal distribution corresponding to the specified confidence level c. Equation 26.2.23 in Abramowitz and Stegun (p. 933) shows how to find this width $x_{p}$ :

$$
c^{\prime}=c / 100
$$

$$
x_{c}=\sqrt{\left[\ln \left(1 / c^{\prime 2}\right)\right]}
$$

$$
x_{p}=x_{c}-\frac{c_{0}+c_{1} x_{c}+c_{2} x_{c}^{2}}{1+d_{1} x_{c}+d_{2} x_{c}{ }^{2}+d_{3} x_{c}{ }^{3}}
$$

$$
\begin{aligned}
& c_{0}=2.515517, c_{1}=0.802853, c_{2}=0.010328 \\
& d_{1}=1.432788, d_{2}=0.189269, d_{3}=0.001308
\end{aligned}
$$

Student's $t$ is then calculated with this formula:

$$
\begin{aligned}
& t=x_{p}+g_{1}\left(x_{p}\right) / d f+g_{2}\left(x_{p}\right) / d f^{2}+g_{3}\left(x_{p}\right) / d f^{3} \\
& \quad+g_{4}\left(x_{p}\right) / d f^{4} \\
& g_{1}(x)=\left(x^{3}+x\right) / 4 \\
& g_{2}(x)=\left(5 x^{5}+16 x^{3}+3 x\right) / 95 \\
& g_{3}(x)=\left(3 x^{7}+19 x^{5}+17 x^{3}-15 x\right) / 384 \\
& g_{4}(x)=\left(79 x^{9}+776 x^{7}+1482 x^{5}-1920 x^{3}-945 x\right) / 92160
\end{aligned}
$$


STANDARD CHECK

(cont)

BIAS CHECK
The standard check is passed with a percent confidence $c$ if

$\left|\left(m_{s t d}-m\right) / \sigma\right| \leq t$

Otherwise, the check is failed.

The average result of $\boldsymbol{n}$ repeated assays on a standard drum is expected to be mave, with a percent confidence level of $c$ (typically 95\%). The actual average found is $m$ with a standard deviation of $\sigma$.

The bias check again involves Student's $t_{1-\alpha / 2}$ at the confidence level $c$, but this time with $n-1$ degrees of freedom.

The bias check is passed if

$\left|m_{\text {ave }}-m\right| \leq t \sigma / \sqrt{[n]}$

Otherwise, the check is failed.

The expected standard deviation from $n$ repeated assays on a standard drum is $\sigma_{\exp }$, with a confidence level of $(1-\alpha)$ (typically 0.95 ). The measured standard deviation is $\sigma$.

This check requires a value of $\chi^{2}(1-\alpha)$ for $d f=n-1$ degrees of freedom. The formula for $\chi 2$ is taken from T. D. Sterling and S. V. Pollack, Introduction to Statistical Data Processing (Prentice-Hall, Inc. Englewood Cliffs, NJ, 1958), p. 289. 
PRECISION CHECK

(cont)
It requires the width $x_{p}$ again, which is calculated as given above.

$\chi^{2}=d f\left\{1-2 /(9 d f)+x_{p} \sqrt{[2 /(9 d f)]}\right\}^{3}$

The check is passed if

$$
\left(\sigma_{\exp } / \sigma\right)^{2}<\chi^{2}
$$

Otherwise, the check is failed. 
INTRODUCTION

Calibration data
The correction between the shuffler's response (corrected count rate $d_{5}$ ) and the $235 \mathrm{U}$ mass $m$ in a drum is established through the calibration process. The calibration process determines the parameters in the expression relating count rate to mass, along with their variances and covariances.

Each type of matrix drum can have a unique set of calibration curves corresponding to different enrichments. Each curve is deduced from measurements on well-characterized calibration drums of a particular enrichment. The ranges of mass and enrichments of the calibration drums must include the ranges of the drums to be assayed later.

If the matrix in a drum is unknown, or known but has not been used in calibration, a "universal" set of calibration curves can be used. This calibration was deduced at Los Alamos from a study with $\mathbf{2 8}$ different matrices and uses the flux monitors to approximately determine the nature of the matrix. A correction is then applied to the measured delayed-neutron count rate. This procedure will not be as accurate as using calibration curves specifically generated for a matrix, but it is available to handle the uncalibrated drums.

Before selecting the "Measurements/Calibration Data" menu option, the proper values of the measu if ient parameters should be set or verified by a technicy i : upervisor. These values should be identical to those tha $t$ will be used later in performing assays on the production drums; the accuracy of the assays depends on this.

Here is a list of parameters that affect the calibration:

General Parameters

Nuclear constants

252 Cf decay constant 


\section{CaLibRation DATA}

(cont)
${ }^{252} \mathrm{Cf}$ decay reference date

235U precursor group fractional yields

$235 U$ precursor decay constants

\section{Drum-Specific Parameters}

Assay parameters

Assay background count time

Source transfer distance

Source transfer acceleration

Source transfer velocity

Nominal number of shuffles per assay

Nominal forward transfer time*

Nominal irradiation time*

Nominal reverse transfer time*

Nominal delayed-neutron count time*

Number of shuffles per assay

Number of scans per shuffle

Scan distance

Scan acceleration

Scan velocity

Pause time at scan's start

Pause time at scan's far point

Rotation

\section{Assay Diagnostics}

Flux monitor use

Reference flux monitor count rate*

Dead time

Normalization

Nomalization factor

Bias Correction

Bias correction parameters

*The parameters with an asterisk can be determined by doing an assay of a calibration drum with the full printout option on. The other numbers on the full printout should be ignored. 


\section{Calibration data}

(cont)

\section{CALIBRATION CURVE}

Unless there are extenuating circumstances, the normalization factor should be one and the bias correction parameters should lead to a bias correction factor of one $\left(a_{2}=0, a_{1}=\right.$ 1 , and $a_{0}=0$ ).

Calibration data can now be taken. There can be as many as 10 drum types. Each type may have as many as $\mathbf{1 0}$ different enrichments. At each enrichment of each type there should be several different $235 \mathrm{U}$ masses.

At each enrichment of each drum type, the measured count rates $d_{5}$ and given $235 \mathrm{U}$ masses $m$ are fitted to this relation:

$$
d_{5}=a_{3} m^{3}+a_{2} m^{2}+a_{1} m+a_{0}
$$

For low masses expected in waste drums the calibration curve should be linear. The $a_{3}$ and $a_{2}$ parameters are then zero. Assays with an empty chamber have shown that $a_{0}$ will also be zero unless there is contamination or holdup within the assay chamber.

If hundreds of grams of ${ }^{235} U$ are inside drums with a moderating matrix, the slope of the calibration curve decreases and the $a_{2}$ parameter will be negative. With kilogram quantities in a moderating matrix, there may be enough multiplication to cause a positive value for $a_{3}$.

Curve-fitting codes for polynomials are widely available, but the code chosen must also generate variances and covariances as well as the parameters themselves. 
ERROR MESSAGES AND RECOVERY

512 and 1024

513 and 1025
The shuffler can detect certain internal faults and provide messages on the printer for study by the operator and supervisors. An error message number is stored with the measurement result in the archival files.

The first set of messages refers to rather general errors and those that might arise from the data analysis. The same errors can be generated in more than one place; an error message in the 500 range in from a general location while those in the 1000 range are from the data analysis. The $\mathbf{2 0 0 0}$ range is for communications with the plant computer; the 4000 range is for problems with the 12-channel scaler board; the 8000 range is for problems with the stepping motor system.

Error in analysis.

An error in the data analysis such as an attempt to divide by zero would generate this message.

The current data are lost. The code is still fully available to perform another measurement. To try to document the problem, a supervisor can turn on the Full Printout and perform a duplicate measurement.

General error.

This message was provided as a general purpose "catch-all" in case a routine was exited with a status value not covered by another error number.

It was not found necessary to implement this error message in the current software, so the message will not appear and no recovery is required. 
514 and 1026

515 and 1027

516 and 1028
Forward overtravel sensor not working.

The code did not receive the expected signal from the forward overtravel sensor. This is most likely to occur during the sensor-test portion of the Safety Check. During normal operation, the overtravel sensor signal is always covered by the Teleflex cable.

Measurement results are unaffected by this error and the impact on the safe use of the shuffler is slight. Even if the source is driven past the forward overtravel switch, the capsule is physically unable to leave the assay chamber. However, if the forward overtravel switch is inoperable, there will be unusual wear on the cable and gears when the capsule strikes the floor of the chamber. The stepping motor may stall, but if it does not, the gears will slip on the cable until the Compumotor Indexer is turned off.

The switch can be tested electrically, positioned closer to the cable, or replaced. Details are in the section on PROXIMITY SWITCHES.

Store sensor not working.

The store sensor is very important and must be working to do assays. The end of the Teleflex cable is carefully brought over this sensor when the source is stored.

The switch can be tested electrically, positioned closer to the cable, or replaced. Details are in the section on PROXIMITY SWITCHES.

Reverse overtravel sensor not working. 
516 and 1028

(cont)

517 and 1029

Delayed- $n$ :utron count time is not positive.

The code did not receive the expected signal from the reverse overtravel sensor. This is most likely to occur during the sensor-test portion of the Safety Check. During normal operation, the overtravel sensor is never covered by the Teleflex cable.

Measurement results are unaffected by this error and the impact on the safe use of the shuffler is slight. Even if the source is driven past the reverse overtravel switch, the capsule is physically unable to leave the storage chamber. However, if the reverse overtravel switch is inoperable, there will be unusual wear on the cable and gears when the capsule strikes the physical stop at the end of the sensor block. The stepping motor may stall, but if it does not, the gears will slip on the cable until the Compumotor Indexer is turned off.

The switch can be tested electrically, positioned closer to the cable, or replaced. Details are in the section on PROXIMITY SWITCHES.

Item ID does not match with a known parameter file.

An item is whatever is being assayed; for this shuffler it is a drum. The drum ID entered by the bar code reader or through the keyboard does not match any of the IDs entered by a technical supervisor in the parameter file. The first characters of an ID are assumed to identify the drum type.

If the ID was entered properly, a technical supervisor can view Edit Parameter/General/Item Type Parameter and add this item type to the list. 
518 and 1030

(cont)

519 and 1031

520 and 1032

\$21 and 1033
The delayed-neutron count time used in the data analysis should always be positive. This time might be nonpositive if there is a problem with the scaler board or communications with the board or with the parallel input port on the Compumotor Indexer.

An assay with the Full Printout will show the earlier point at which the count is not positive. A supervisor can use the suboptions under Diagnostics to exercise the scaler board under simple conditions to study the problem.

Irradiate time is not positive.

This error has much in common with error 4102 because the timer and the counter share the same electrical hardware.

A supervisor can use the suboptions under Diagnostics to exercise the scaler board's timer under simple conditions to study the problem.

Background count time is not positive.

The background count time should always be positive. This time might be nonpositive if there is a problem with the scaler board or communications with the board or with the parallel input port on the Compumotor Indexer.

A supervisor can use the suboptions under Diagnostics to exercise the scaler board under simple conditions to study the problem.

Flux monitor count rate $\mathbf{= 0 . 0}$.

The flux monitor count rate during irradiation of a drum should not be zero. 
521 and 1033

(cont)

522 and 1034

523 and 1035

524 and 1036
The scaler board or the detector tube circuitry may have malfunctioned. A supervisor can exercise the flux monitors through the Diagnostics option.

Calculated DSUM $=0.0$.

In the data analysis a sum is computed for use in correcting the delayed-neutron count rate for slight irregularities in the timing of events. This sum should never be zero. No known conditions exist that would make this sum zero, but a check is made of its value before it is used as a divisor.

A programmer can wise a debugger on the data analysis routine to examine values being used.

Number of counts from delayed-neutron bank not positive.

The number of counts in the delayed-neutron banks should always be positive. This number might be nonpositive if there is a problem with the scaler board or communications with the board or with the parallel input port on the Compumotor Indexer.

An assay with the Full Printout will show the earlier point at which the count is not positive. A supervisor can use the suboptions under Diagnostics to exercise the scaler board under simple conditions to study the problem.

Ratio of counts from delayaj-neutron banks out of range.

The counts from delayed-neutron banks 1 and 3 are added and compared to the sum of counts in banks 2 and 4. This error arises if the ratio is outside the range specified in the parameter file for the drum being studied. 
524 and 1036

(cont)

525 and 1037

526 and 1038

527 and 1039
An assay with the Full Printout on will show all the data involved in this check. If there is noise in one or more banks, the large number of counts will reveal it.

Number of counts from flux monitor bank not positive.

The number of counts from the flux monitors should always be positive. This count might be nonpositive if there is a problem with the scaler board or communications with the board or with the parallel input port on the Compumotor Indexer.

An assay with the Full Printout will show the earlier point at which the count is not positive. A supervisor can use the suboptions under Diagnostics to exercise the scaler board under simple conditions to study the problem.

Ratio of flux monitor banks out of range.

The count from flux monitor one is divided by the count from flux monitor two. This error arises if the ratio is outside the range specified in the parameter file for the drum being studied.

An assay with the Full Printout on will show all the data involved in this check. If there is noise in one or more banks, the large number of counts will reveal it.

Nominal DSUM is less than or equal to 0.0.

A nominal value of DSUM is computed as part of every assay, using nominal values of timing parameters. The value is always positive; a nonpositive value is an error. The nominal DSUM is purely calculational in nature; measured data are not used. 
527 and 1039

(cont)

528 and 1040

529 and 1041

530 and 1042
These parameters should be examined for correctness: nominal forward transfer time, nominal irradiation time, nominal reverse transfer time, nominal count time, nominal number of shuffles per assay, nuclear parameters (delayed neutron yields and decay constants).

Enrichment value not within range.

The enrichment of a drum to be assayed must fall between two enrichments found in the calibration data for that type of drum. Extrapolation will not be done, so an error is generated.

The enrichments for that type of drum can be reviewed as part of the calibration parameters. The first calibration curve must have the lowest enrichment and the rest of the enrichments should follow in ascending order.

Interpolation convergence error.

A generalized, versatile interpolation routine is used to interpolate the drum's mass from the two calibration curves with enrichments flanking the drum's enrichment. A numerical, iterative process is used that in principle can fail to converge. If convergence is not found after the number of iterations built into the code, this message is generated.

This code uses simple linear interpolation and the convergence should never be a problem. A programmer can use a debugger on the interpolation routine to examine values being used.

Lamp not operating. 


\section{USBS MANUAL}

530 and 1042

(cont)

531 and 1043

532 and 1044

533 and 1045
During a Safety Check, the user is asked to check the proper operation of the status lamps. If the user indicates a problem, this error is generated.

A lamp might be burned out or signals might not reach it from the computer and the output parallel port of the Compumotor Indexer.

Door switch failure.

During Safety Check the user is asked to open the doors and then to verify that the doors are indeed open. If the doors' proximity sensor does not agree that the doors are open, this error is generated.

The switch can be tested electrically, positioned closer to the cable, or replaced. Details are in the section on PROXIMITY SWITCHES.

Door-closed sensor malfunction.

During Safety Check the user is asked to close the doors and then to verify that the doors are indeed closed. If the doors' proximity sensor does not agree that the doors are closed, this error is generated.

The switch can be tested electrically, positioned closer to the cable, or replaced. Details are in the section on PROXIMITY SWITCHES.

Fatal hardware error.

This is a potential error message from the collect data routine to handle unspecified errors. It is not implemented. 
534 and 1046

535 and 1047

536 and 1048
Bias measurement failed.

The bias part of a Bias and Precision check failed the statistical test. If the test is performed at the $95 \%$ confidence level, statisticians tell us that the test should fail 1 time out of 20 ( $5 \%$ of the time).

Relevant values are given on the printer and can be checked. Parameters controlling the test can be checked in the parameter file under Edit Parameters/General/Bias and Precision Parameters.

Precision measurement failed.

The precision part of a Bias and Precision check failed the statistical test. If the test is performed at the $95 \%$ confidence level, statisticians tell us that the test should fail 1 time out of 20 ( $5 \%$ of the time).

Relevant values are given on the printer and can be checked. Parameters controlling the test can be checked in the parameter file under Edit Parameters/General/Bias and Precision Parameters.

Standard check failed.

An assay with a standard drum performed as part of the Standard Check was outside the range specified in the parameter file. If the test is performed at the $95 \%$ confidence level, statisticians tell us that the test should fail 1 time out of 20 (5\% of the time). If the test is attempted on a drum other than the standard drum, the test is likely to fail. 
536 and 1048

(cont)

537 and 1049

538 and 1050

539 and 1051
Parameters controlling the test can be checked in the parameter file under Edit Parameters/General/Standard Check Parameters.

Reverse transfer error.

If the Compumotor does not complete the reverse transfer motion (rapidly returning the source to its store position) in a reasonable time, this error message is generated. There is a serious hardware or electrical malfunction that should be corrected before assays should continue.

A supervisor can exercise the equipment through the Diagnostics option of the main menu.

Empty check failure.

An assay with an empty chamber performed as part of the Standard Check was outside the range specified in the parameter file. If a drum or a certain amount of holdup material was present in the chamber, the test is failed.

Parameters controlling the test can be checked in the parameter file under Edit Parameters/General/Standard Check Parameters.

Cycle correction factor error.

A cycle correction factor is calculated as part of every assay to correct measured count rates for small irregularities in the timing of the various portions of an assay. A maximum deviation from one is set in the parameter file and this error message is generated when the cycle correction factor is out of that range. 
539 and 1051

(cont)

540 and 1052

541 and 1053

542 and 1054

543 and 1055
If the range is set too small, false "alarms" can be generated. Nominal times and the number of shuffles must be as close as possible to actual values, as shown on Full Printouts.

After the proper setting of nominal values, the cycle correction factor should routinely be within $0.5 \%$ of one.

Normalization factor not 1.

This is not so much an error as a reminder that this assay was performed with a normalization factor applied to the count rate just before the interpolations from the calibration curves. The normalization factor should normally be one and have no effect on the assays.

Not used.

Archive full—oldest data not transmitted.

The number of measurements that will be retained in the archives is set in the parameter file. Normally these results are immediately transmitted to the plant computer, when the archive is full, a new result is added by dropping the oldest result. If the oldest result has not yet been transmitted, it is not to be dropped; a new assay is simply made impossible to do and this error generated.

The size of the archive can be increased by a supervisor. When communications with the plant computer revive, old data not yet transmitted will be sent and new measurements can proceed.

Assay not okay-bias measurement failed.

The previous bias measurement was outside the expected range and thus the bias check was failed. A supervisor has 
543 and 1055

(cont)

544 and 1056

545 and 1057

2048

2049 taken an assay without first passing the bias check, so the assay is flagged with this status code.

Assay not okay — standard check failed.

An assay as part of a previous standard check was outside the expected range and thus the standard check was failed. A supervisor has taken an assay without first passing the standard check, so the assay is flagged with this status code.

Assay not okay - safety check failed.

A safety check was previously failed. A supervisor has taken an assay without first passing the safety check, so the assay is flagged with this status code.

The next set of errors deals with the process control database.

Network down.

If the shuffler's computer cannot communicate with the network, this message is generated.

If the network is in fact not down, the communication link between the computer and the network must be faulty.

Bad item.

For this shuffler, an item is a waste drum. This message states that the ID of a drum was not acceptable. 
2049

(cont)

2050

2051

2052

2053

2054
The given ID may have been in error, the ID may not exist in a database.

Item is in the wrong place in the process.

If a drum should not be assayed in the shuffler at this stage in the process, this message is generated.

The drum should be sent to its proper place or the database altered.

Data were not stored.

A measurement was completed but the result could not be transmitted. The data will remain in the shuffler's archive with this message attached.

Data stored, but item is at the process hold point.

This flags a measurement result that the drum has been assayed but is now at a hold point in the process.

Data stored, but the item is rejected.

This flags a measurement result that the drum has been assayed, but the drum is now rejected from the process.

Process computer database error.

This is a general-purpose signal that an undefined error has occurred with the process-computer database. 


\section{USERS MANUAL}

2054

(cont)

4096

4097

4098
The next set of messages deals with the 12-channel scaler board.

Scaler responded successfully.

This is more of a status message than an error message. It is ustd by the shuffler's computer internally to give assurance that actions with the scaler board are correct.

Invalid channel number for scaler.

If the code attempts to deal with a channel number outside the range of valid numbers, this message is generated.

A coding error has been encountered that must be corrected.

Not enough bytes sent to scaler.

When the scaler board does not receive the proper number of bytes from the shuffler computer, it returns this message to the shuffler and passes it along to the user.

When the shuffler code is started, it attempts to communicate with the scaler NIM. If power to the NIM is off, this communication is impossible and this error will be generated the next time the scaler NIM is accessed. Exit the code and give power to the scaler NIM; then restart the code.

Communications may be tested easily by a technical supervisor who has access to the Diagnostic/Count with No Californium Motion option. A count time of $1 \mathrm{~s}$ will quickly force communications with the scaler. The display will show the counts in the channels if the communication was successful; otherwise, an error message will appear. 
4098

(cont)

4099

4100

4101

4102
If the problem persists, turning the power off to the NIM bin, exiting the shuffler code, turning power on to the NIM, and restarting the code may clear the problem; the quickest way to find out is to use the Count with No Californium Motion option described in the previous paragraph. If the problem persists, the most likely cause is with the hardware of the communication link. A malfunction of the scaler board is another possibility.

Scaler number is unknown.

An unrecognized parameter number has been sent to the scaler.

A coding error has occurred and must be corrected.

Invalid port number for scaler.

An attempt has been made to communicate with the scaler board through the wrong serial port.

A coding error has occurred and must be corrected.

Scaler port not open.

An attempt has been made to communicate with the scaler without first opening the proper port.

A coding error has occurred and must be corrected.

Error in communicating with scaler.

This is a general-purpose message that could not be more specific about the communications problem. 
4102

(cont)

4103

8192

8193

8194
The board should be tested with standard diagnostic electronics tools.

Problem with scaler.

A problem has arisen that could not be identified.

The communications link could be at fault, a coding error may have arisen, or there may be a hardware problem on the scaler board.

The next set of messages concerns the Compumotor stepping motor system.

Compumotor responded successfully.

This is more of a status message than an error message. It indicates that NO error occurred during an operation requested by the computer.

Compumotor did not respond.

If the Compumotor does not signal the completion of an action in a reasonable time period, this error is set.

The cause might be a failure in the Compumotor system or a requested action with a poor choice of parameters. For example, if a forward transfer is requested but the acceleration and velocity are very low, the action will not be completed in the usual small amount of time.

Invalid Compumotor register. 
8194

(cont)

8195

8196

8197

8198
The shuffler code passes values into registers within the Compumotor Indexer, these values are used by the Compumotor code. If the specified register is outside the range of valid values, this error is generated.

A coding error has occurred within the shuffler's code. That portion of the code must be corrected.

Invalid motor axis.

If the shuffler code requests action by a Compumotor motor axis that does not exist, this error is generated.

A coding error has occurred within the shuffler's code. That portion of the code must be corrected.

Invalid write to Compumotor.

If a communication is not understood by the Compumotor, it returns a signal that stimulates this error message.

The communication link may be faulty or a programming error may have sent an unintelligible message.

Move did not complete.

A transfer (forward or reversi') of the source was not accomplished in a reasonable time.

There could be a malfunction in the Compumotor system or the acceleration or velocity parameters for performing the move are set too low.

Scan did not complete. 
8198

(cont)

8199

8200

$\mathbf{8 2 0 1}$

8202
The source is scanned the length of the drum during irradiation. If the scan was not performed in a reasonable time, this error message is generated.

There could be a malfurction in the Compumotor system or the acceleration or velocity parameters for performing the scan are set too low.

Step ratio invalid.

This signals an incorrect value of a parameter internal to the code. Shufflers always use a fixed ratio and this error message will not occur.

Invalid port number for Compumotor.

The serial port assigned to the Compumotor Indexer is invalid.

The shuffler code must be corrected to use the correct port.

Compumotor port not open.

Communication with the Compumotor Indexer is noi occurring.

The cable connecting the computer and the Inciexer could be faulty, the Indexer might be in an incorrect mode to receive the communication, or the serial communication board irs the computer might be faulty.

Error in finding home. 
8202

(cont)

3203

8204

8205
The Compumotor system could not position the free end of the Teleflex cable above the store proximity switch, thus placing the source in its store position.

A supervisor can exercise this operation in a simple way through the Diagnostic option. The switch may be faulty, the Compumotor Indexer may be malfunctioning, or the code inside the Compumotor may have been altered.

Error in communicating to Compumotor.

This is a general-purpose error when communications with the Compumotor are unintelligible.

Cables, serial boards, and external noise could cause the problem.

Compumotor error.

When the Compumotor signals that an error has occurred and it could not be deciphered into a specific problem, this error message is generated.

The Compumotor can be exercised through its own control panel or through the shuffler's Jiagnostics options to try to isolate the problem.

Door opeı-source movement not allowed.

The Compumotor was commanded to move the source, but the doors' proximity switch indicated that the doors were open. The code in the Indexer will not move the source. This is an error condition. 
8205

(cont)

8206

8207

PROXIMITY SWITCHES
If the doors are in fact closed, the proximity switch could be at fault, as could be the circuitry between the switch, the Indexer, and the shuffler's computer.

Bad branch register value.

If the Indexer is told to run a routine of its internal code starting at a nonexistent point, this error is generated.

Either there is a coding error in the shuffler's code or the communications failed to transmit the value correctly.

Move aborted - overtravel sensor reached.

If a move was terminated by the overtravel sensor before the move was completed, this error is generated.

Probably the user sets a distance for the move that could not be completed. Otherwise a malfunction of the Indexer could be suspected.

A proximity switch can be tested by moving the cable (or doors) off the switch and activating the switch with a piece of metal (such as a bolt or screwdriver).

There should be $12 \mathrm{~V}$ supplied to the switch from the NIM bin power supply. If this is not present, the switch cannot function.

A voltmeter across the output of the switch will show its operation. With iron in contact with the switch, the voltage should be $0 \mathrm{~V}$; with iron removed, the voltage should be $6 \mathrm{~V}$. If the switch does not respond properly, it must be replaced. 
PROXIMITY SWITCHES

(cont)
SCALER BOARD LAMPS
If the switch does respond properly to the iron test but not to the cable (or doors), undo the locking nut and screw the switch closer to the cable (or doors). Only one half turn at a time should be used before testing the switch with the cable (or doors).

The position of the switch must be carefully set. The switch screws into the mounting block; the tip should just come in contact with the Teleflex cable or the doors. The Teleflex cable slides on the switch, but the switch must not obstruct the movement of the cable. A little wear on the tip is normal, but if the cable has worn through the black plastic tip, the switch was too close to the cable and the switch should be replaced.

The scaler boand receives TTL signals from the Amplifier/ SCAs inside the electronics junction box. These signals are used to flash the LEDs on the front of the scaler NIM while they are being counted in 10 scaler channels.

The channel 0 lamp should be on continuously because this is responding to a $10 \mathrm{kHz}$ clock inside the NIM. Channels 1 through 8 receive signals from delayed-neutron banks. Channels 9 and 10 receive signals from flux monitors. Channel 11 is not used and its lamp should remain off.

At very low count rates, the individual flashes of the LEDs are apparent, but at even moderately high count rates the LEDs are on continuously. This continuous illumination should not occur when a drum and the ${ }^{252} \mathrm{Cf}$ source are not present in the assay region. The spontaneously emitted neutrons from a drum may or may not be at a sufficient rate to turn the LEDs on continuously. 
SCALER BOARD LAMPS (cont)

INDEXER COMMUNICATIONS

MOTOR DRIVER FAULT
If a detector bank has failed, its corresponding LED could be either on all the time or off all the time, depending on the mode of failure. In either case, a problem is indicated. The data analysis process checks for unusual detector bank counts and should also indicate a problem that the LED can identify more clearly. A technical supervisor can use the Diagnostic/Count with No Californium Motion suboption to check the operation of the detector banks.

If moisture has entered an electronics junction box and is causing high-voltage arcing, there will be noise in the signal from that junction box. The data analysis process with the LEDs could identify this problem and both parts of the Standard Check are likely to be failed. A technical supervisor can use the Diagnostic/Count with No Californium Motion suboption to check the operation of the detector banks.

If communication with the Indexer is not working properly, check these settings under SETUP/RS232: baud rate = 9600,1 stop bit, 7 data bits, even parity, DTE. Under SETUP/REMOTE, TEXT should be set to OFF.

In unusual circumstances the Indexer may be asked to move the source in a manner that leads to a fault in the motor driver, an example might be to move the source at very high speed well beyond an overtravel switch. If this occurs, recover by (1) unplugging the power to the motor driver, using the power cord going into the Power/Signal box on the outside of the assay chamber, (2) turning power OFF and then $\mathrm{ON}$ to the Indexer, and then (3) plugging the power cord back into the Power/Signal box for the motor driver. 
LUBRICATION

VOLTAGE TESTS

JUNCTION BOX DESICCANT
The Teleflex cable should be oiled with Pro Tech Metal Treatment every six months if a shuffler is used regularly; every year if used irregularly. This can be done by wiping Pro Tech into the forward overtravel sensor while moving the shaft of the motor by hand (motor power off) or while the motor is driving the cable at low speed (use the Move Californium option of the Diagnostics menu). This does not directly apply oil to the source capsule end of the cable, but it is not worth the additional radiation exposure to oil this portion.

If the source and cable are removed from the shuffler and placed in a temporary cask, the capsule and the nearby portion of the cable can be swiped with oil while being removed or replaced in the shuffler.

Test points are on the Los Alamos National Laboratory NIMs, and these should be checked once a month.

The NIM bin itself has test points that should be checked once a month.

The electronics junction boxes have desiccant in capsules that screw into the lid. These capsules are revealed after removing the cavity cover plate, the source spindle, and the cavity liner. It is not necessary to remove the junction box to replace the desiccant.

The central spot in the clear top of the capsule shows the relative humidity of the desiccant within the capsule. If the spot is blue, the humidity is low; if the spot is pink, the humidity is high. High humidity may lead to arcing across some high-voltage components and generate noise in the signal lines. A pink spot does not necessarily mean that 
JUNCTION BOX DESICCANT (cont) arcing will occur, but it is prudent to replace the desiccant capsule.

Around the centrai spot are indicators of three levels of the ambient relative humidity. A pink sector shows the relative humidity outside the capsule and inside the junction box. 


\section{ASSAY TIMINGS AND ESTIMATION OF PARAMETERS}

The Purpose

The Model
When a fixed amount of time is available to complete an assay, how much of the time will be spent on background counting and how much on irradiation and delayed-neutron counting must be decided. This appendix will outline the procedure for making this decision. This is condensed from the analysis given in the Los Alamos National Laboratory report LA-12105 (May 1991) by P. M. Rinard, "Shuffler Instruments for the Nondestructive Assay of Fissile Materials."

The criterion is to select times that minimize the relative precision of the delayed-neutron count.

The number $D$ of delayed neutrons counted in a time interval $T_{C}$ is the measured number of counts $C$ minus an estimate of the background counts $B$. The $B$ is estimated from a previous background count; if $B_{b}$ is the number of counts in the background count time $T_{b}$, then

$D=C-B=C-B_{b}\left(T_{c} / T_{b}\right)$

The relative precision squared of $D$ is

$\sigma_{D} / D=\left(\sigma_{C}^{2}+\sigma_{B}^{2}\right) / D$

The variance of $C$ is $\sigma_{C}^{2}=D+B_{b}\left(T_{c} / T_{b}\right)$; that of $B$ is $B_{b}\left(T_{c} / T_{b}\right)$. The relative precision squared of $D$ is thus

$$
\left(\sigma_{D} / D\right)^{2}=\left[D+B_{b}\left(T_{c} / T_{b}\right)+B_{b}\left(T_{c} / T_{b}\right)^{2}\right] / D^{2}
$$


The Model

(cont)
This is better expressed in terms of rates and times by using $D=d T_{c}$ and $B=b T_{c}$, where $d$ is the delayed-neutron rate and $b$ is the background rate. In terms of these rates, the square of the relative precision is

$$
\left(\sigma_{D} / D\right)^{2}=\left(d T_{c}+b T_{c}+b T_{c}^{2} / T_{b}\right) /\left(d T_{c}\right)^{2}
$$

A parameter search is made from this equation, using known values when possible and keeping unknown parameters within reasonable ranges.

The variation of the relative precision with changes in the parameters is rather slow and many choices are nearly equivalent. Prudence dictates that a set of parameters is favored if it uses fewer shuffles per assay, thus driving the hardware less often. It is considered better to sacrifice some of the relative precision for lower shuffle numbers. 

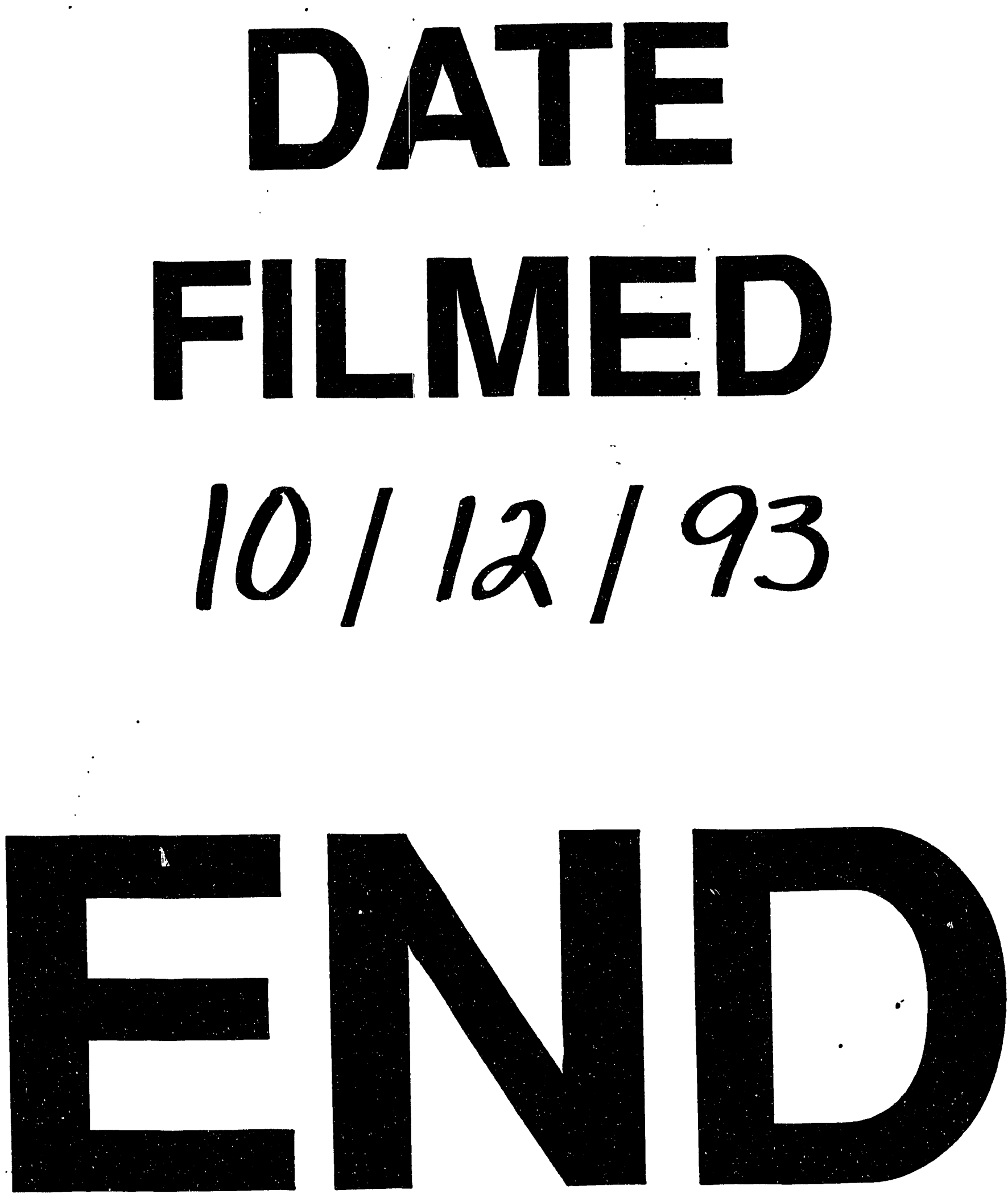
